Supporting Information

Pennelliisides A-C, 2,3,4-Trisubstituted Acyl Glucoses Isolated from Solanum pennellii

Tenki Nakashima, Yurika Nambu, Yutaka Inoue, Masimbula Rishni, and

Hideyuki Matsuura

Research Faculty of Agriculture, Hokkaido University, Sapporo 060-8589, Japan 


\section{Pennelliisides A-C, 2,3,4-Trisubstituted Acyl Glucoses Isolated from Solanum pennellii}

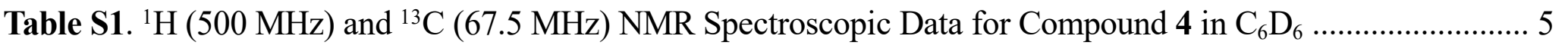

Table S2. ${ }^{1} \mathrm{H}(500 \mathrm{MHz})$ and ${ }^{13} \mathrm{C}(67.5 \mathrm{MHz}) \mathrm{NMR}$ Spectroscopic Data for Compound 5 in $\mathrm{C}_{6} \mathrm{D}_{6} . \ldots \ldots \ldots \ldots \ldots \ldots \ldots . . . . . . . . . .6$

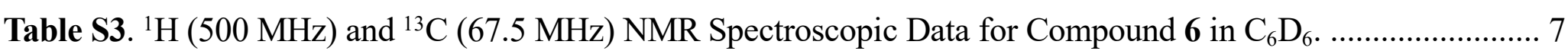

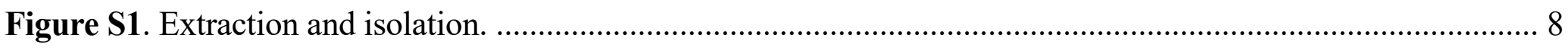

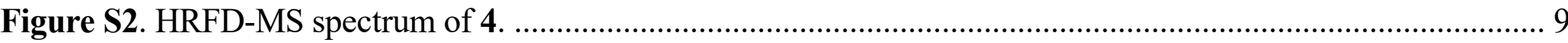

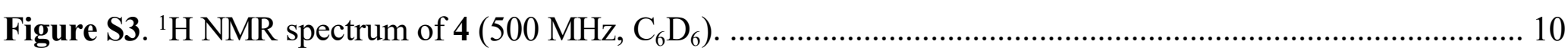

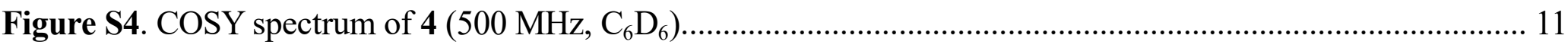

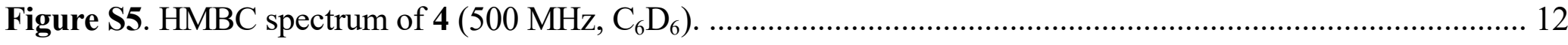

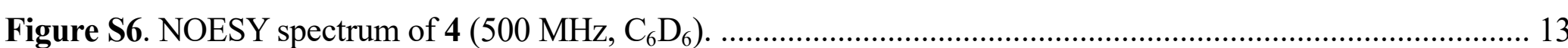

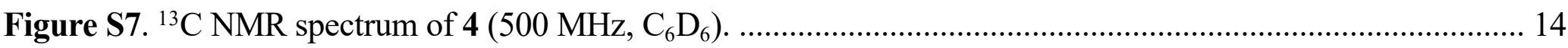

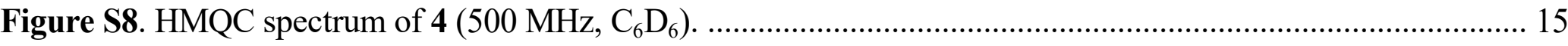

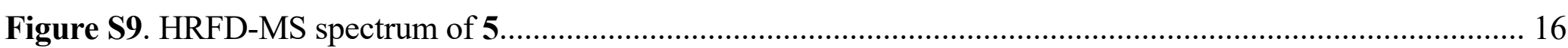

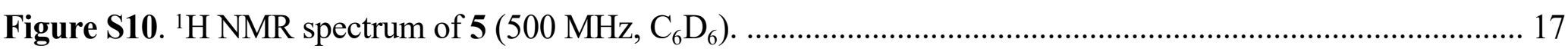

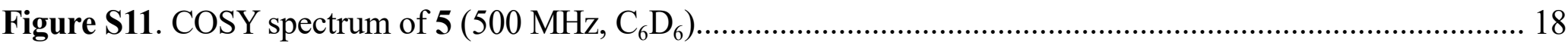

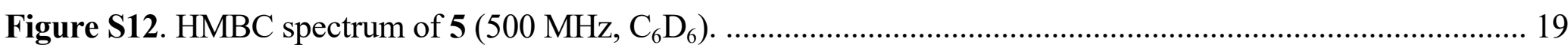

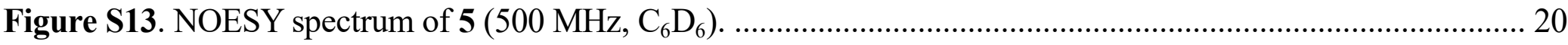

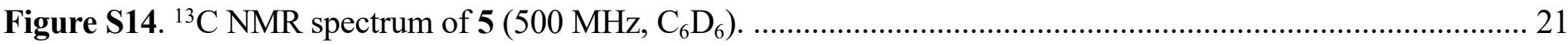

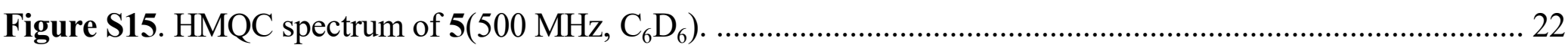

Figure S16. ${ }^{1} \mathrm{H}$ NMR spectrum of 8-methylnonanoate (7) $(270 \mathrm{MHz}, \mathrm{CDCl} 3)$. ............................................... 23

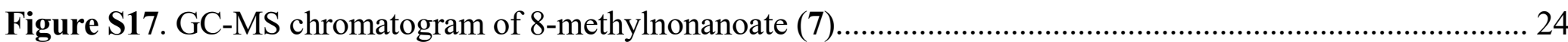


Figure S18. Fragmentation pattern of the MS peak for 8-methylnonanoate (7)

having Rt. of $12.1 \mathrm{~min}$ in the chromatogram of Figure S17

Figure S19. GC-MS chromatograph of methylated acyl moiety of 5.

Figure S20. Fragmentation pattern of the MS peak for methylated acyl moiety of 5

having Rt. of $12.4 \mathrm{~min}$ in the chromatogram of Figure S19.

Figure S21. HRFD-MS spectrum of 6. 28

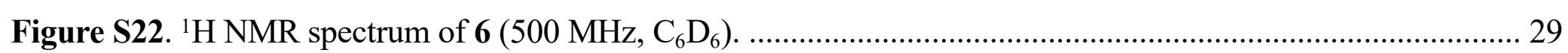

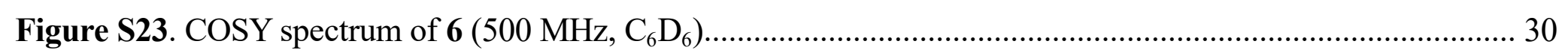

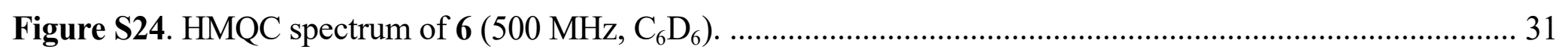

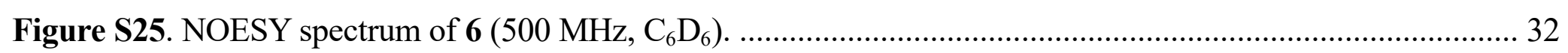

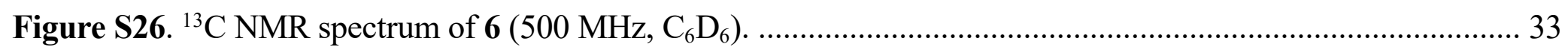

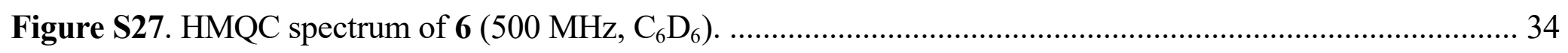

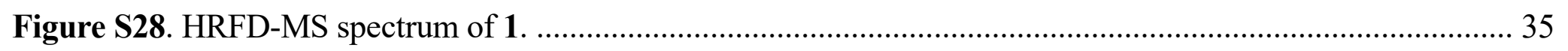

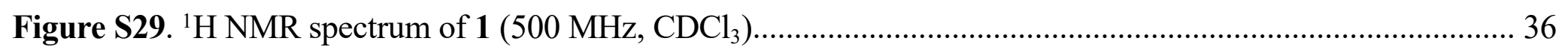

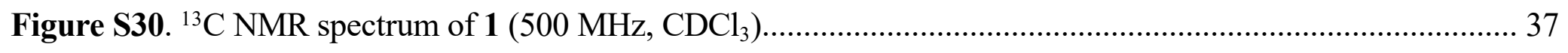

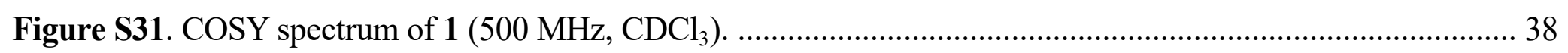

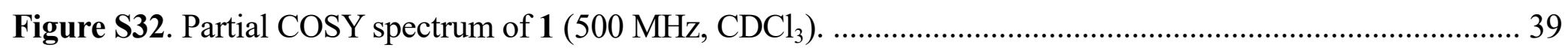

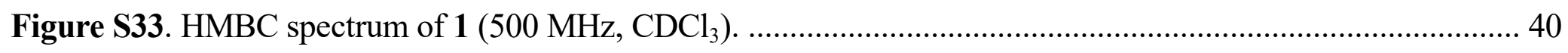

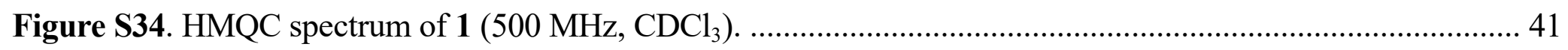

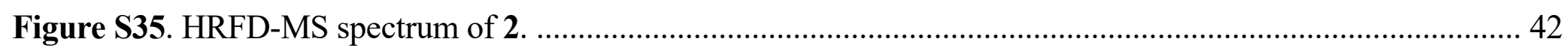

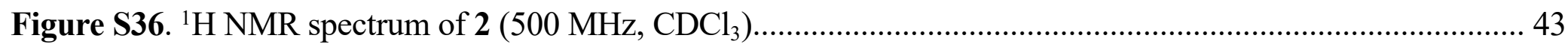

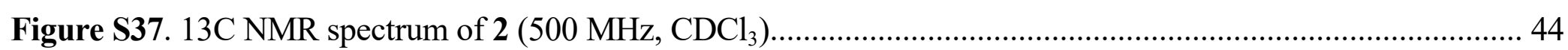




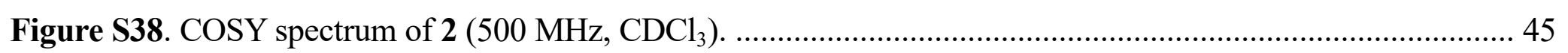

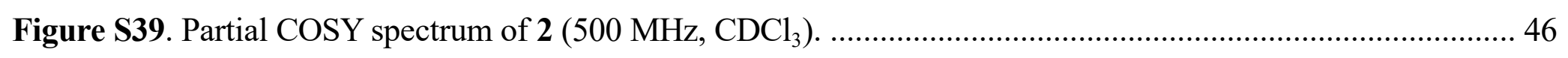

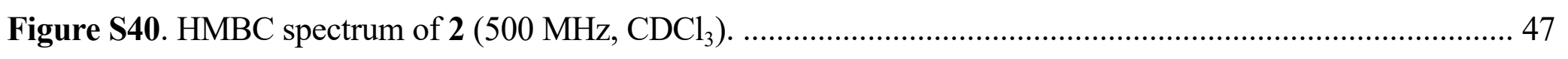

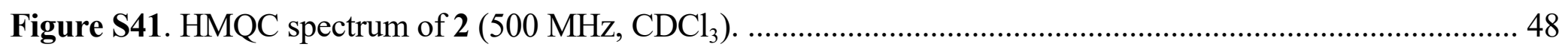

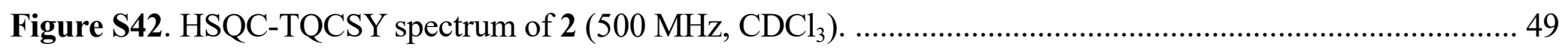

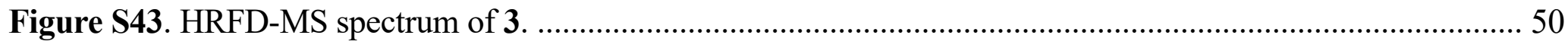

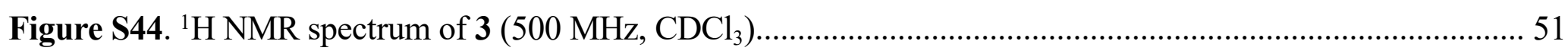

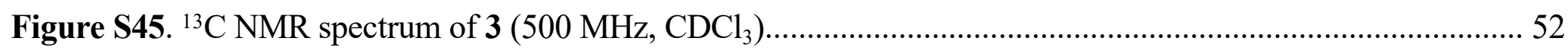

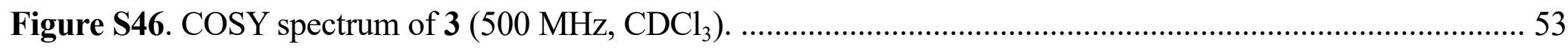

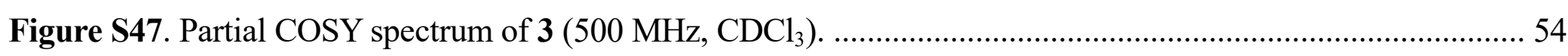

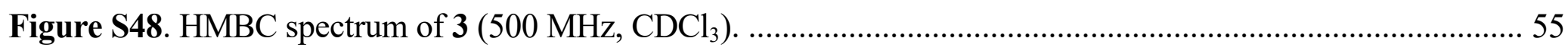

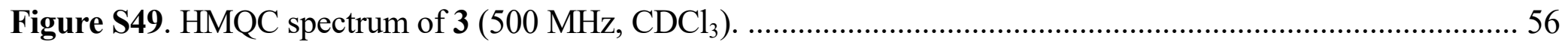

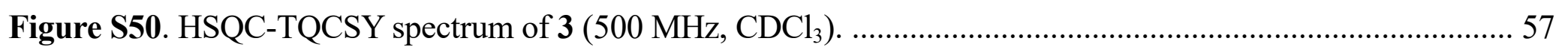

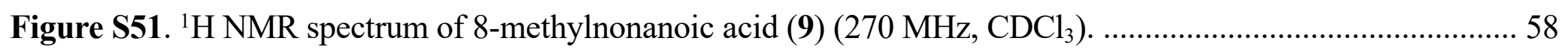

Figure S52. ${ }^{1} \mathrm{H}$ NMR spectrum of 6-methylheptanoic acid (14) $\left(270 \mathrm{MHz}, \mathrm{CDCl}_{3}\right)$....................................59

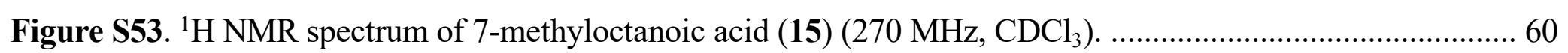


Table S1. ${ }^{1} \mathrm{H}(500 \mathrm{MHz})$ and ${ }^{13} \mathrm{C}(67.5 \mathrm{MHz})$ NMR Spectroscopic Data for Compound 4 in $\mathrm{C}_{6} \mathrm{D}_{6}$

\begin{tabular}{|c|c|c|c|c|}
\hline & position & $\delta \mathrm{c}$ & type & $\delta \mathrm{H}$, mult. $(J$ in $\mathrm{Hz})$ \\
\hline \multirow{6}{*}{$\begin{array}{l}\text { glucose } \\
\text { moiety }\end{array}$} & 1 & 99.5 & $\mathrm{CH}$ & $4.36, \mathrm{~d}(6.3)$ \\
\hline & 2 & 72.9 & $\mathrm{CH}$ & $5.45, \mathrm{dd}(8.6,6.3)$ \\
\hline & 3 & 71.3 & $\mathrm{CH}$ & $5.43, \mathrm{dd}(9.3,8.6)$ \\
\hline & 4 & 69.2 & $\mathrm{CH}$ & $5.29, \mathrm{dd}(9.3,9.1)$ \\
\hline & 5 & 73.5 & $\mathrm{CH}$ & $3.39, \mathrm{~m}$ \\
\hline & 6 & 69.2 & $\mathrm{CH}_{2}$ & $3.47, \mathrm{~m}$ \\
\hline \multirow{8}{*}{$\begin{array}{l}\text { benzyl } \\
\text { moiety }\end{array}$} & $1 \mathrm{a}^{\prime}$ & \multirow{2}{*}{70.7} & \multirow{2}{*}{$\mathrm{CH}_{2}$} & $4.74, \mathrm{~d}(12.8)$ \\
\hline & $1 b^{\prime}$ & & & $4.73, \mathrm{~d}(12.8)$ \\
\hline & $2^{\prime}$ & 137.4 & $\mathrm{C}$ & \\
\hline & $3^{\prime}$ & 128.2 & $\mathrm{CH}$ & $7.24, d(7.5)$ \\
\hline & $4^{\prime}$ & $128.1-127.4$ & $\mathrm{CH}$ & 7.11-7.19, m \\
\hline & $5^{\prime}$ & $128.1-127.4$ & $\mathrm{CH}$ & $7.07, \mathrm{t}(7.3)$ \\
\hline & $6^{\prime}$ & $128.1-127.4$ & $\mathrm{CH}$ & 7.11-7.19, m \\
\hline & $7^{\prime}$ & 128.2 & $\mathrm{CH}$ & $7.24, d(7.5)$ \\
\hline \multirow{12}{*}{$\begin{array}{l}\text { acyl } \\
\text { moiety }\end{array}$} & A-1 & 174.4 & $\mathrm{C}$ & \\
\hline & A-2 & 33.9 & $\mathrm{CH}$ & $2.41, \mathrm{~m}(7.0)$ \\
\hline & A-3 & $18.5-18.7$ & $\mathrm{CH}_{3}$ & $1.07, \mathrm{~d}(7.0)$ \\
\hline & A-4 & $18.5-18.7$ & $\mathrm{CH}_{3}$ & $1.07, \mathrm{~d}(7.0)$ \\
\hline & B-1 & 175.5 & $\mathrm{C}$ & \\
\hline & B-2 & 33.9 & $\mathrm{CH}$ & $2.39, \mathrm{~m}(7.1)$ \\
\hline & B-3 & $18.5-18.7$ & $\mathrm{CH}_{3}$ & $1.06, \mathrm{~d}(7.1)$ \\
\hline & B-4 & $18.5-18.7$ & $\mathrm{CH}_{3}$ & $1.06, \mathrm{~d}(7.1)$ \\
\hline & $\mathrm{C}-1$ & 174.6 & $\mathrm{C}$ & \\
\hline & $\mathrm{C}-2$ & 33.9 & $\mathrm{CH}$ & $2.30, \mathrm{~m}(7.0)$ \\
\hline & $\mathrm{C}-3$ & $18.6^{\mathrm{a}}$ & $\mathrm{CH}_{3}{ }^{\mathrm{a}}$ & $1.01 . \mathrm{d}(7.0)^{\mathrm{b}}$ \\
\hline & $\mathrm{C}-4$ & $18.5^{\mathrm{a}}$ & $\mathrm{CH}_{3}{ }^{\mathrm{a}}$ & $0.97, \mathrm{~d}(7.0)^{b}$ \\
\hline \multirow{8}{*}{$\begin{array}{l}\text { benzyl } \\
\text { moiety }\end{array}$} & $1 a^{\prime \prime}$ & \multirow{2}{*}{73.2} & \multirow{2}{*}{$\mathrm{CH}_{2}$} & $4.33, \mathrm{~d}(12.3)$ \\
\hline & $1 b^{\prime \prime}$ & & & $4.30, \mathrm{~d}(12.3)$ \\
\hline & $2^{\prime \prime}$ & 138.3 & $\mathrm{C}$ & \\
\hline & $3^{\prime \prime}$ & 128.2 & $\mathrm{CH}$ & 7.27, d (7.3) \\
\hline & $4^{\prime \prime}$ & $128.1-127.4$ & $\mathrm{CH}$ & 7.11-7.19, m \\
\hline & $5^{\prime \prime}$ & $128.1-127.4$ & $\mathrm{CH}$ & $7.07, \mathrm{t}(7.3)$ \\
\hline & $6^{\prime \prime}$ & $128.1-127.4$ & $\mathrm{CH}$ & 7.11-7.19, m \\
\hline & $7^{\prime \prime}$ & 128.2 & $\mathrm{CH}$ & 7.27, d (7.3) \\
\hline
\end{tabular}


Table S2. ${ }^{1} \mathrm{H}(500 \mathrm{MHz})$ and ${ }^{13} \mathrm{C}(67.5 \mathrm{MHz})$ NMR Spectroscopic Data for Compound 5 in $\mathrm{C}_{6} \mathrm{D}_{6}$

\begin{tabular}{|c|c|c|c|c|}
\hline & position & $\delta c$ & type & $\delta \mathrm{H}$, mult. $(J$ in $\mathrm{Hz})$ \\
\hline \multirow{6}{*}{ glucose moiety } & 1 & 99.4 & $\mathrm{CH}$ & $4.39, \mathrm{~d}(7.7)$ \\
\hline & 2 & 71.3 & $\mathrm{CH}$ & $5.46, \mathrm{dd}(9.0,7.7)$ \\
\hline & 3 & 72.8 & $\mathrm{CH}$ & $5.49, \mathrm{dd}(9.2,9.0)$ \\
\hline & $y_{4}$ & 69.3 & $\mathrm{CH}$ & $5.31, \mathrm{dd}(9.2,9.1)$ \\
\hline & 5 & 73.5 & $\mathrm{CH}$ & $3.43, \mathrm{~m}$ \\
\hline & 6 & 69.1 & $\mathrm{CH}_{2}$ & $3.48, \mathrm{~m}$ \\
\hline \multirow{8}{*}{ benzyl moiety } & $1 \mathrm{a}^{\prime}$ & \multirow{2}{*}{69.9} & \multirow{2}{*}{$\mathrm{CH}_{2}$} & $4.75, \mathrm{~d}(12.2)$ \\
\hline & $1 b^{\prime}$ & & & $4.46, \mathrm{~d}(12.2)$ \\
\hline & $2^{\prime}$ & 137.3 & $\mathrm{C}$ & \\
\hline & $3^{\prime}$ & $128.5-127.0$ & $\mathrm{CH}$ & $7.24, \mathrm{~d}(7.7)$ \\
\hline & $4^{\prime}$ & $128.5-127.0$ & $\mathrm{CH}$ & 7.11-7.19, m \\
\hline & $5^{\prime}$ & $128.5-127.0$ & $\mathrm{CH}$ & $7.07, \mathrm{t}(7.3)$ \\
\hline & $6^{\prime}$ & $128.5-127.0$ & $\mathrm{CH}$ & 7.11-7.19, m \\
\hline & $7^{\prime}$ & $128.5-127.0$ & $\mathrm{CH}$ & $7.24, \mathrm{~d}(7.7)$ \\
\hline \multirow{18}{*}{ acyl moiety } & A-1 & 174.5 & $\mathrm{C}$ & \\
\hline & A-2 & 33.8 & $\mathrm{CH}$ & $2.45, \mathrm{~m}(6.9)$ \\
\hline & A-3 & $18.5^{\mathrm{a}}$ & $\mathrm{CH}_{3}$ & $1.12, \mathrm{~d}(6.9)$ \\
\hline & A-4 & $18.6^{\mathrm{a}}$ & $\mathrm{CH}_{3}$ & $1.12, \mathrm{~d}(6.9)$ \\
\hline & B-1 & 172.3 & $\mathrm{C}$ & \\
\hline & B-2 & 33.9 & $\mathrm{CH}_{2}$ & $2.22, \mathrm{t}(7.6)$ \\
\hline & B-3 & 24.7 & $\mathrm{CH}_{2}$ & $1.58, \mathrm{~m}$ \\
\hline & B-4 & 29.5 & $\mathrm{CH}_{2}$ & $1.20-1.15, \mathrm{~m}$ \\
\hline & B-5 & 29.0 & $\mathrm{CH}_{2}$ & $1.20-1.15, \mathrm{~m}$ \\
\hline & B-6 & 27.0 & $\mathrm{CH}_{2}$ & $1.20, \mathrm{~m}$ \\
\hline & B-7 & 38.9 & $\mathrm{CH}_{2}$ & $1.10, \mathrm{~m}$ \\
\hline & B-8 & 27.8 & $\mathrm{CH}$ & $1.47, \mathrm{~m}$ \\
\hline & B-9 & 22.4 & $\mathrm{CH}_{3}$ & $0.88, \mathrm{~d}(6.5)$ \\
\hline & B-10 & 22.4 & $\mathrm{CH}_{3}$ & $0.88, \mathrm{~d}(6.5)$ \\
\hline & C-1 & 174.6 & $\mathrm{C}$ & \\
\hline & $\mathrm{C}-2$ & 33.7 & $\mathrm{CH}$ & $2.34, \mathrm{~m}(6.9)$ \\
\hline & $\mathrm{C}-3$ & $18.5^{b}$ & $\mathrm{CH}_{3}$ & $1.05 . d(6.9)^{c}$ \\
\hline & $\mathrm{C}-4$ & $18.4^{\mathrm{b}}$ & $\mathrm{CH}_{3}$ & $1.00, \mathrm{~d}(6.9)^{\mathrm{c}}$ \\
\hline \multirow{8}{*}{ benzyl moiety } & $1 a^{\prime \prime}$ & \multirow{2}{*}{73.1} & \multirow{2}{*}{$\mathrm{CH}_{2}$} & $4.35, \mathrm{~d}(12.3)$ \\
\hline & $1 b^{\prime \prime}$ & & & $4.31, \mathrm{~d}(12.3)$ \\
\hline & $2^{\prime \prime}$ & 138.2 & $\mathrm{C}$ & \\
\hline & $3^{\prime \prime}$ & $128.5-127.0$ & $\mathrm{CH}$ & $7.27, \mathrm{~d}(7.3)$ \\
\hline & $4^{\prime \prime}$ & $128.5-127.0$ & $\mathrm{CH}$ & $7.11-7.19, \mathrm{~m}$ \\
\hline & $5^{\prime \prime}$ & $128.5-127.0$ & $\mathrm{CH}$ & $7.07, \mathrm{t}(7.3)$ \\
\hline & $6 "$ & $128.5-127.0$ & $\mathrm{CH}$ & $7.11-7.19, \mathrm{~m}$ \\
\hline & $7 "$ & $128.5-127.0$ & $\mathrm{CH}$ & $7.27, \mathrm{~d}(7.3)$ \\
\hline
\end{tabular}


Table S3. ${ }^{1} \mathrm{H}(500 \mathrm{MHz})$ and ${ }^{13} \mathrm{C}(67.5 \mathrm{MHz})$ NMR Spectroscopic Data for Compound 6 in $\mathrm{C}_{6} \mathrm{D}_{6}$

\begin{tabular}{|c|c|c|c|c|}
\hline & position & $\delta c$ & type & $\delta \mathrm{H}$, mult. $(J$ in $\mathrm{Hz})$ \\
\hline \multirow{6}{*}{$\begin{array}{l}\text { glucose } \\
\text { moiety }\end{array}$} & 1 & 99.4 & $\mathrm{CH}$ & $4.39, \mathrm{~d}(7.3)$ \\
\hline & 2 & 71.3 & $\mathrm{CH}$ & 5.47, dd $(9.7,7.3)$ \\
\hline & 3 & 72.8 & $\mathrm{CH}$ & $5.49, \mathrm{dd}(9.7,9.6)$ \\
\hline & 4 & 69.2 & $\mathrm{CH}$ & $5.32, \mathrm{dd}(9.6,9.1)$ \\
\hline & 5 & 73.5 & $\mathrm{CH}$ & $3.43, \mathrm{~m}$ \\
\hline & 6 & 69.1 & $\mathrm{CH}_{2}$ & $3.48, \mathrm{~m}$ \\
\hline \multirow{8}{*}{$\begin{array}{l}\text { benzyl } \\
\text { moiety }\end{array}$} & $1 a^{\prime}$ & \multirow{2}{*}{69.9} & \multirow{2}{*}{$\mathrm{CH}_{2}$} & $4.75, \mathrm{~d}(12.1)$ \\
\hline & $1 b^{\prime}$ & & & $4.45, \mathrm{~d}(12.1)$ \\
\hline & $2^{\prime}$ & 137.3 & $\mathrm{C}$ & \\
\hline & $3^{\prime}$ & $128.2-127.1$ & $\mathrm{CH}$ & $7.24, \mathrm{~d}(7.5)$ \\
\hline & $4^{\prime}$ & $128.2-127.1$ & $\mathrm{CH}$ & 7.11-7.19, m \\
\hline & $5^{\prime}$ & $128.2-127.1$ & $\mathrm{CH}$ & $7.07, \mathrm{t}(7.3)$ \\
\hline & $6^{\prime}$ & $128.2-127.1$ & $\mathrm{CH}$ & 7.11-7.19, m \\
\hline & $7^{\prime}$ & $128.2-127.1$ & $\mathrm{CH}$ & $7.24, \mathrm{~d}(7.5)$ \\
\hline \multirow{16}{*}{$\begin{array}{c}\text { acyl } \\
\text { moiety }\end{array}$} & A-1 & 174.5 & $\mathrm{C}$ & \\
\hline & A-2 & 33.8 & $\mathrm{CH}$ & $2.45, \mathrm{~m}(7.4)$ \\
\hline & A-3 & $18.6^{\mathrm{a}}$ & $\mathrm{CH}_{3}$ & $1.14-0.98, \mathrm{~d}(7.4)$ \\
\hline & $\mathrm{A}-4$ & $18.5^{\mathrm{a}}$ & $\mathrm{CH}_{3}$ & $1.14-0.98, \mathrm{~d}(7.4)$ \\
\hline & B-1 & 172.3 & $\mathrm{C}$ & \\
\hline & B-2 & 33.9 & $\mathrm{CH}_{2}$ & $2.22, \mathrm{t}(7.3)$ \\
\hline & B-3 & 24.7 & $\mathrm{CH}_{2}$ & $1.59, \mathrm{~m}$ \\
\hline & B-4 & 29.1 & $\mathrm{CH}_{2}$ & $1.20, \mathrm{~m}$ \\
\hline & B-5-B-7 & 29.4- 29.1 & $\mathrm{CH}_{2}$ & $1.35-1.75, \mathrm{~m}$ \\
\hline & B-8 & 31.8 & $\mathrm{CH}_{2}$ & $1.22, \mathrm{~m}$ \\
\hline & B-9 & 22.6 & $\mathrm{CH}_{2}$ & $1.28, \mathrm{~m}$ \\
\hline & B-10 & 13.9 & $\mathrm{CH}_{3}$ & $0.90, \mathrm{t}(6.8)$ \\
\hline & C-1 & 174.6 & $\mathrm{C}$ & \\
\hline & $\mathrm{C}-2$ & 33.7 & $\mathrm{CH}$ & $2.34 \mathrm{~m}(6.9)$ \\
\hline & $\mathrm{C}-3$ & $18.5^{b}$ & $\mathrm{CH}_{3}$ & $1.14-0.98, \mathrm{~d}(6.9)$ \\
\hline & C-4 & $18.4^{\mathrm{b}}$ & $\mathrm{CH}_{3}$ & $1.14-0.98, \mathrm{~d}(6.9)$ \\
\hline \multirow{8}{*}{$\begin{array}{l}\text { benzyl } \\
\text { moiety }\end{array}$} & $1 a^{\prime \prime}$ & \multirow{2}{*}{73.1} & \multirow{2}{*}{$\mathrm{CH}_{2}$} & $4.35, \mathrm{~d}(12.3)$ \\
\hline & $1 b^{\prime \prime}$ & & & $4.31, \mathrm{~d}(12.3)$ \\
\hline & $2^{\prime \prime}$ & 138.2 & $\mathrm{C}$ & \\
\hline & $3^{\prime \prime}$ & $128.2-127.1$ & $\mathrm{CH}$ & $7.27, \mathrm{~d}(7.5)$ \\
\hline & $4^{\prime \prime}$ & $128.2-127.1$ & $\mathrm{CH}$ & 7.11-7.19, m \\
\hline & $5^{\prime \prime}$ & $128.2-127.1$ & $\mathrm{CH}$ & $7.07, \mathrm{t}(7.3)$ \\
\hline & $6^{\prime \prime}$ & $128.2-127.1$ & $\mathrm{CH}$ & 7.11-7.19, m \\
\hline & $7^{\prime \prime}$ & $128.2-127.1$ & $\mathrm{CH}$ & 7.27, d (7.5) \\
\hline
\end{tabular}


Leaves and stems (1.7 kg) of tomato (Solanum pennellii)

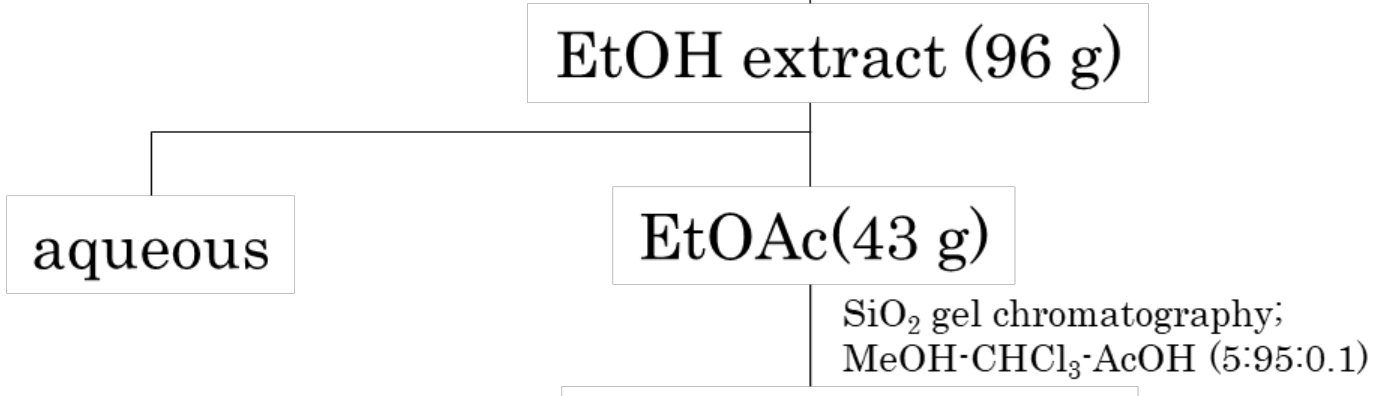

\section{Benzylation (29 g)}

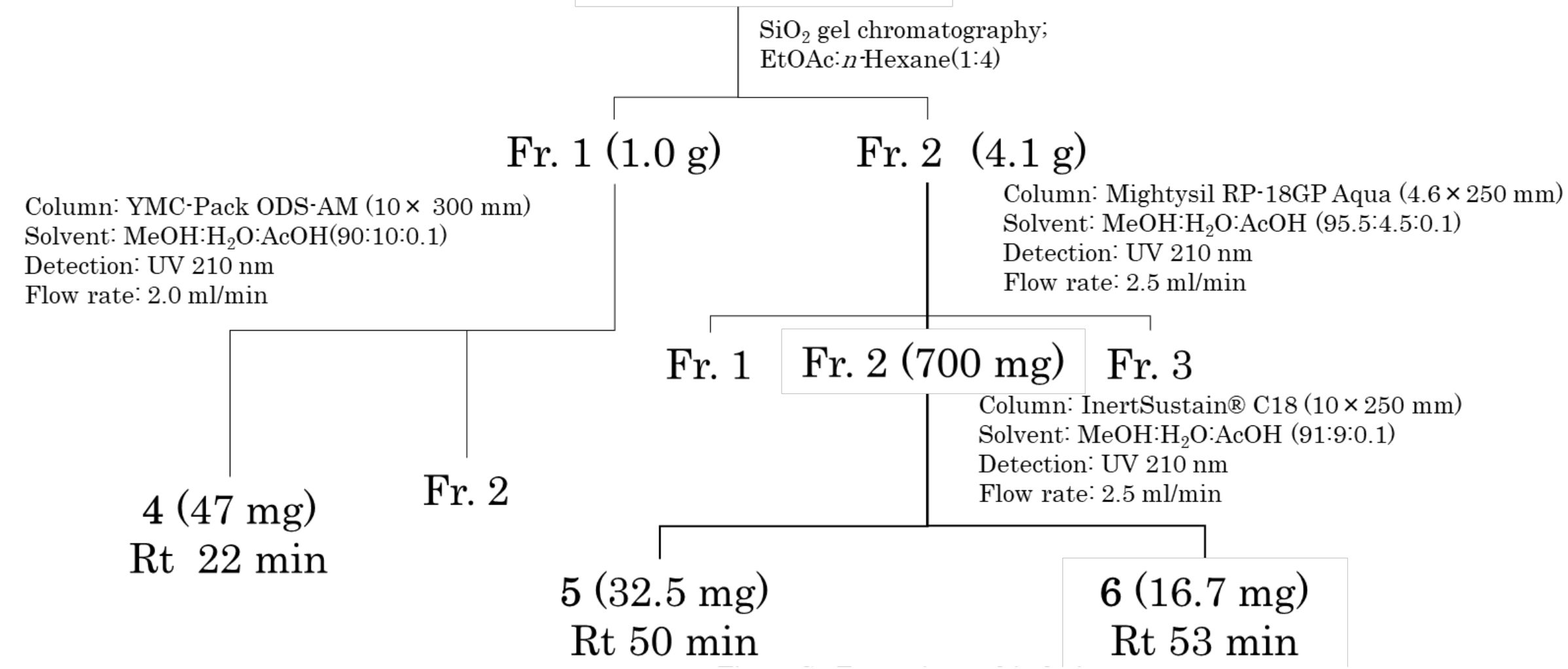

Figure S1. Extraction and isolation. 


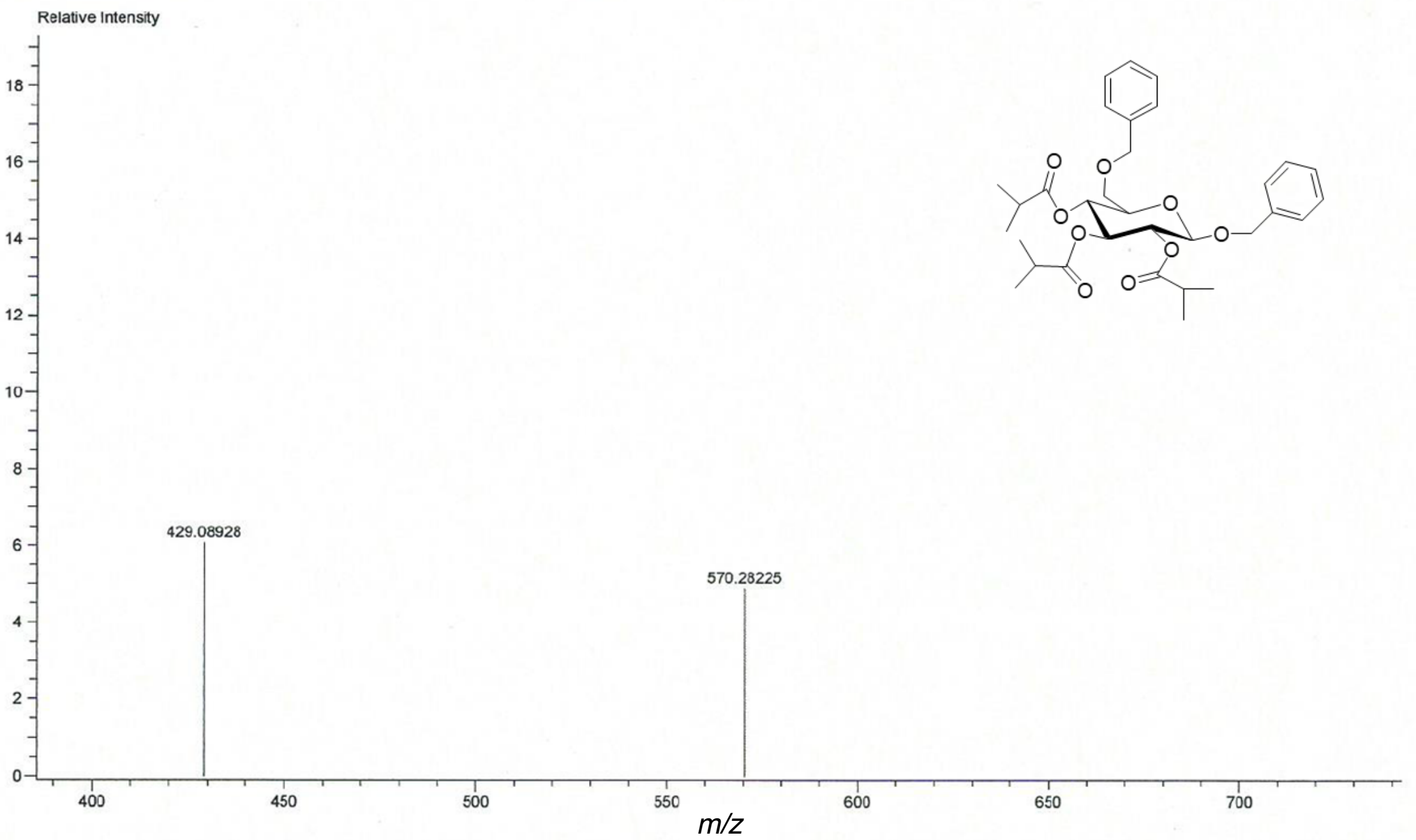

Figure S2. HRFD-MS spectrum of 4. 


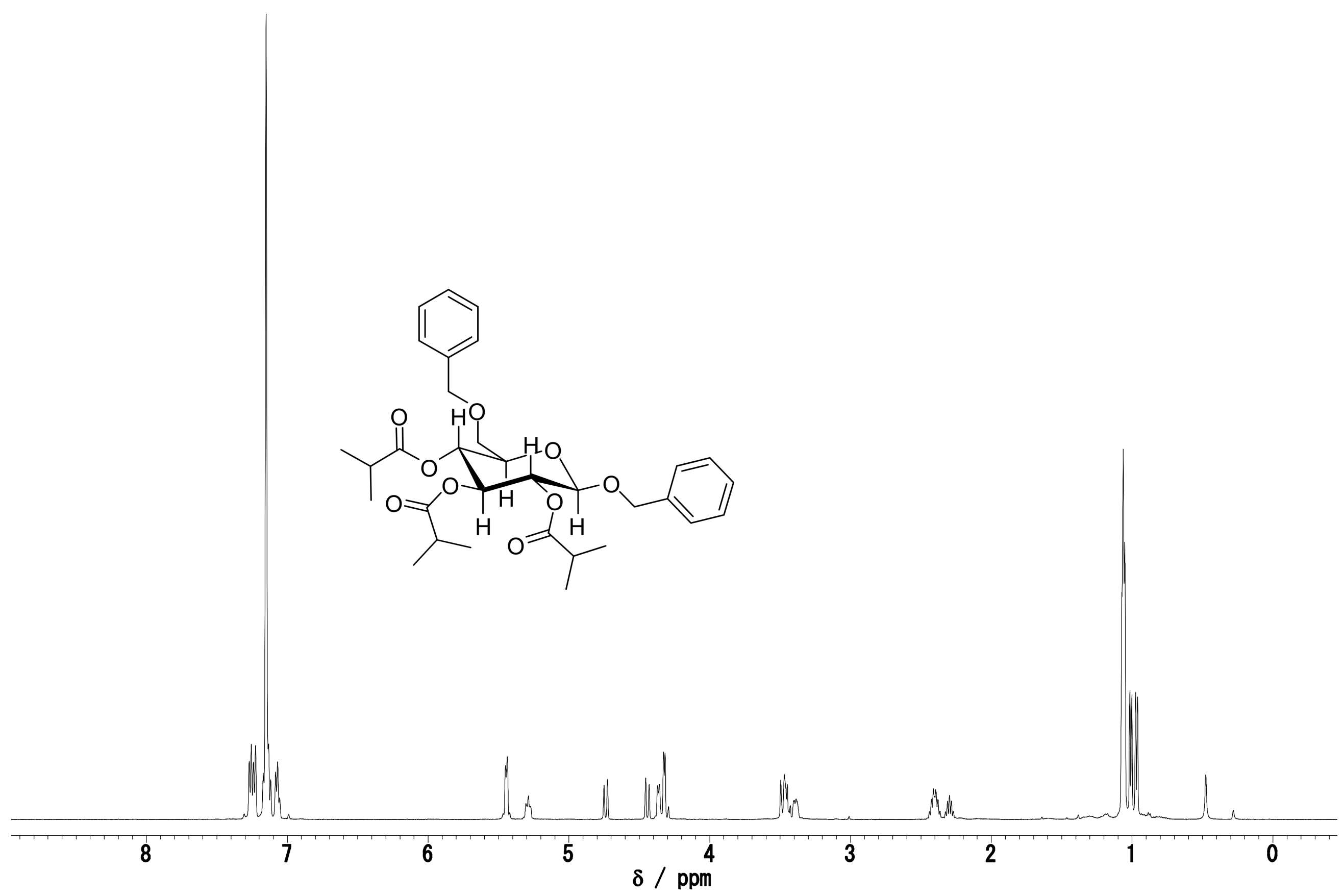

Figure S3. ${ }^{1} \mathrm{H}$ NMR spectrum of $4\left(500 \mathrm{MHz}, \mathrm{C}_{6} \mathrm{D}_{6}\right)$. 

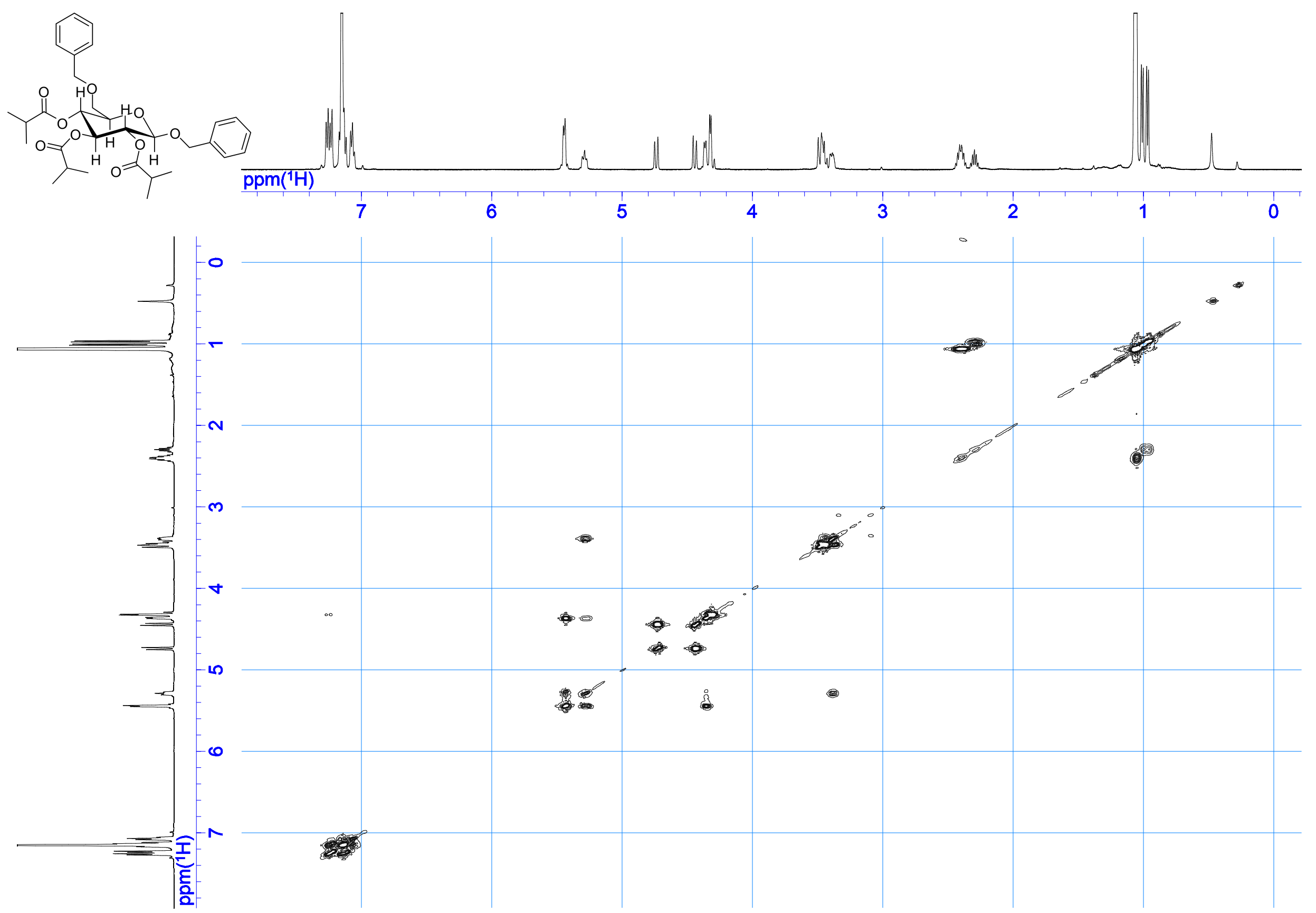

Figure S4. COSY spectrum of $4\left(500 \mathrm{MHz}, \mathrm{C}_{6} \mathrm{D}_{6}\right)$.

11 

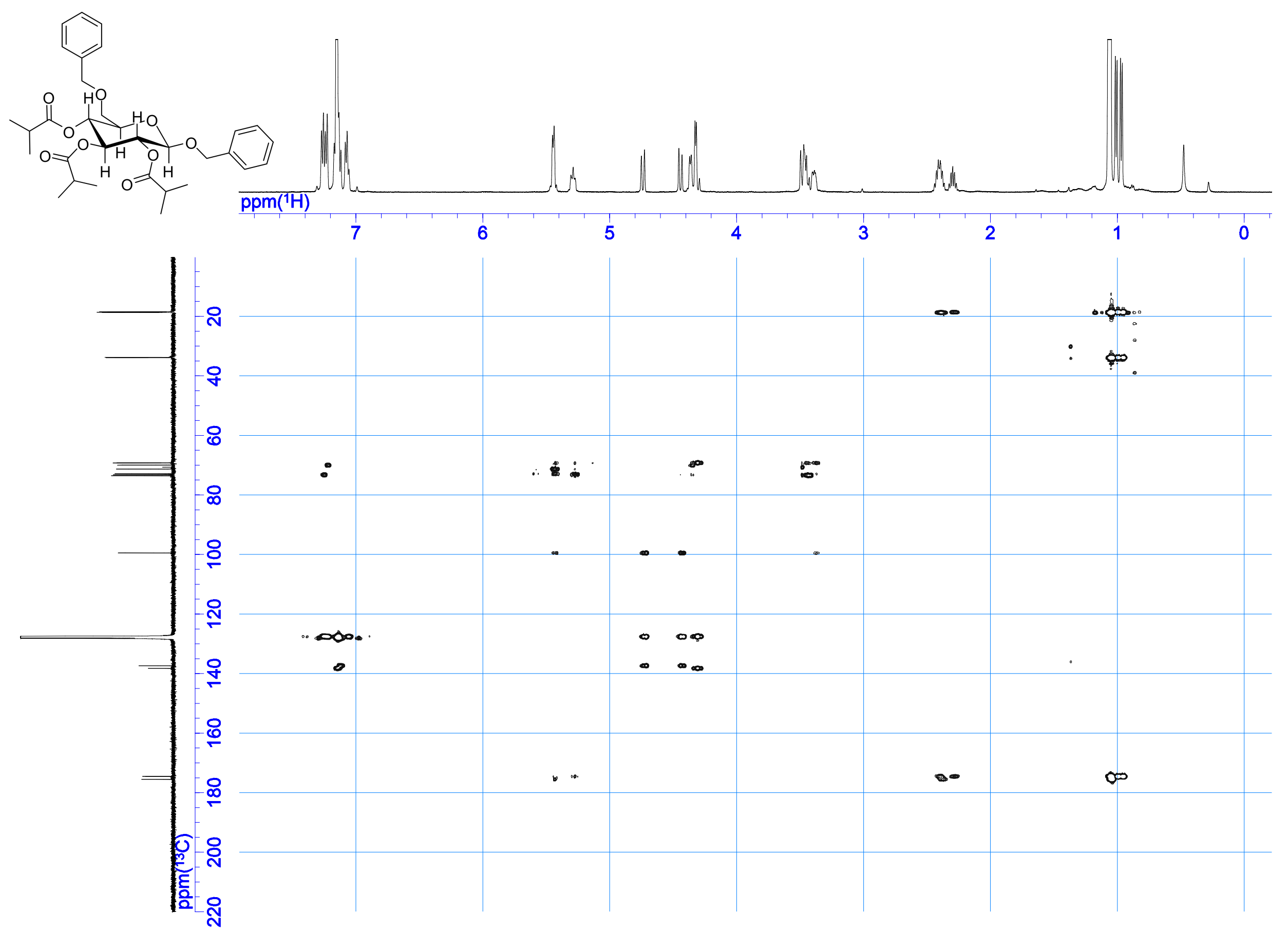

Figure S5. HMBC spectrum of $4\left(500 \mathrm{MHz}, \mathrm{C}_{6} \mathrm{D}_{6}\right)$. 

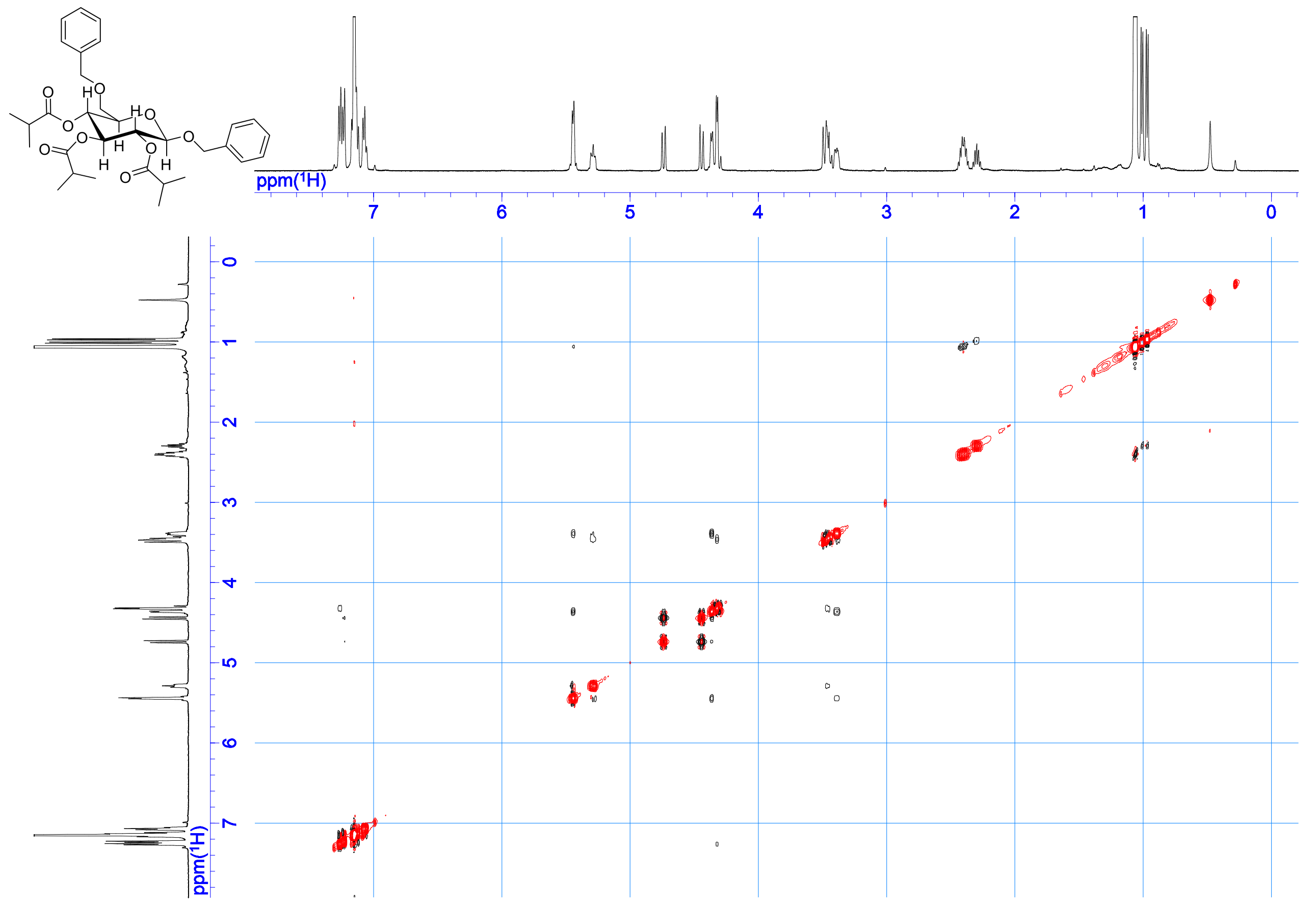

Figure S6. NOESY spectrum of $4\left(500 \mathrm{MHz}, \mathrm{C}_{6} \mathrm{D}_{6}\right)$.

13 


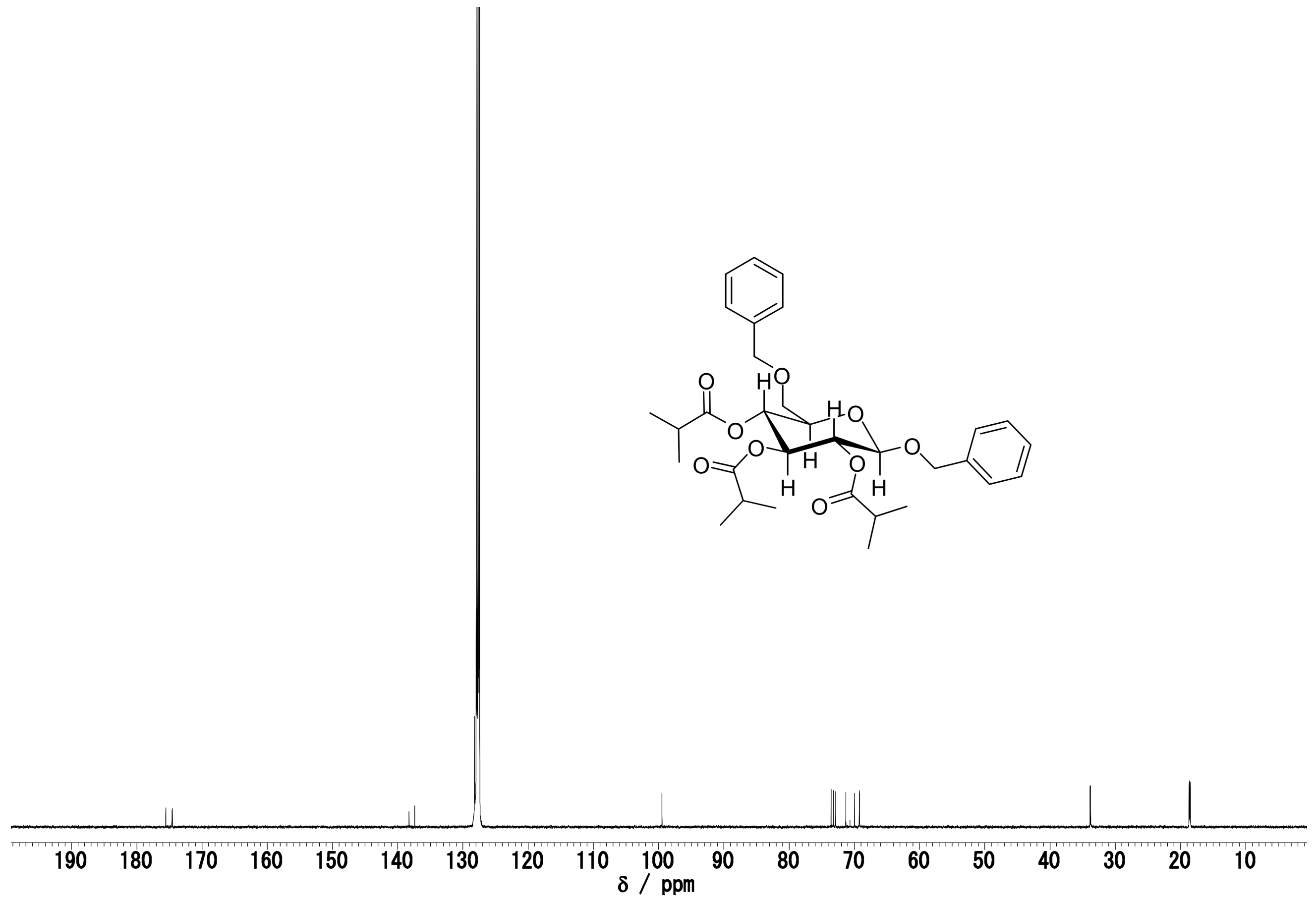

Figure S7. ${ }^{13} \mathrm{C}$ NMR spectrum of $4\left(500 \mathrm{MHz}, \mathrm{C}_{6} \mathrm{D}_{6}\right)$. 

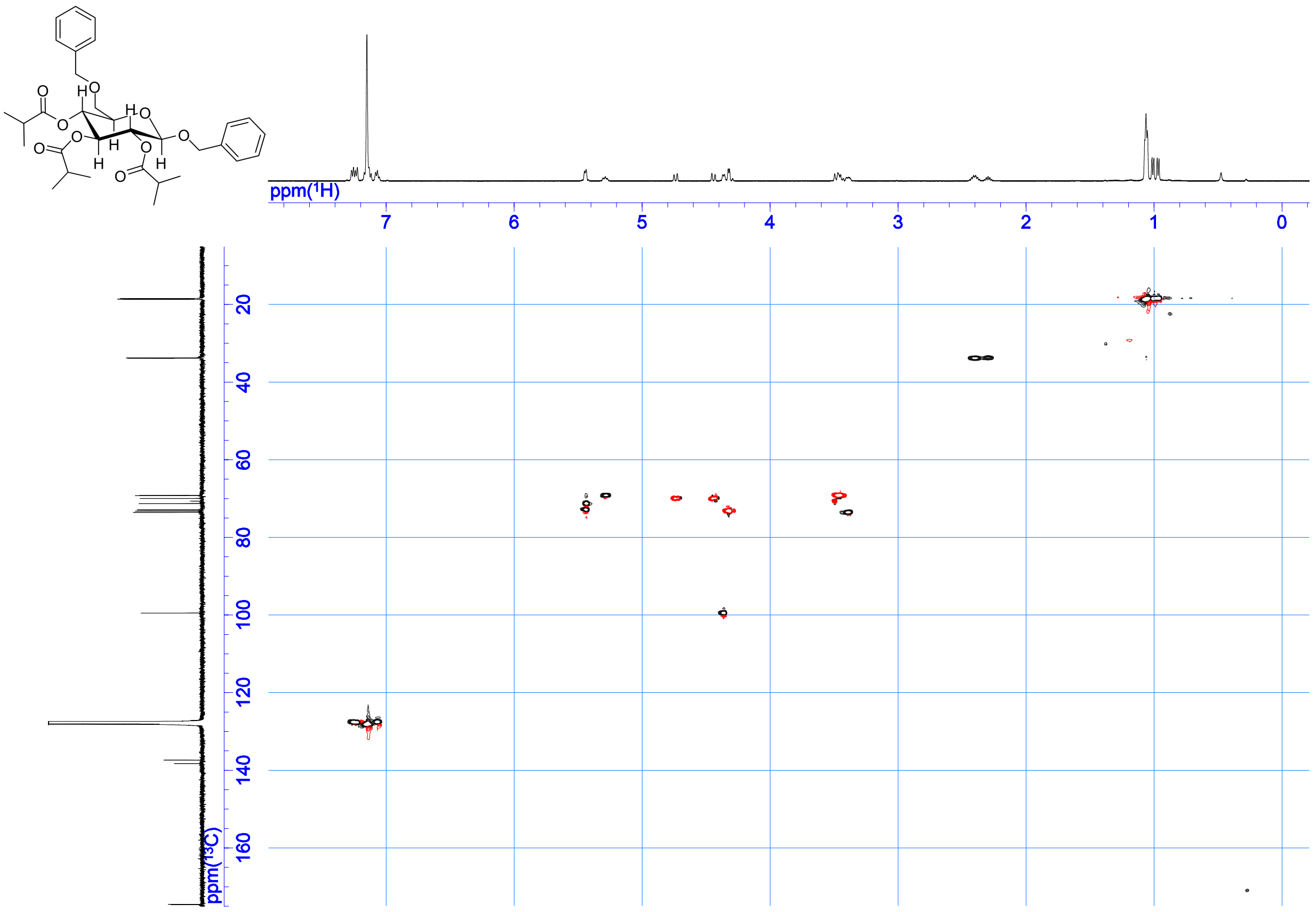

Figure S8. HSQC spectrum of $4\left(500 \mathrm{MHz}, \mathrm{C}_{6} \mathrm{D}_{6}\right)$.

15 


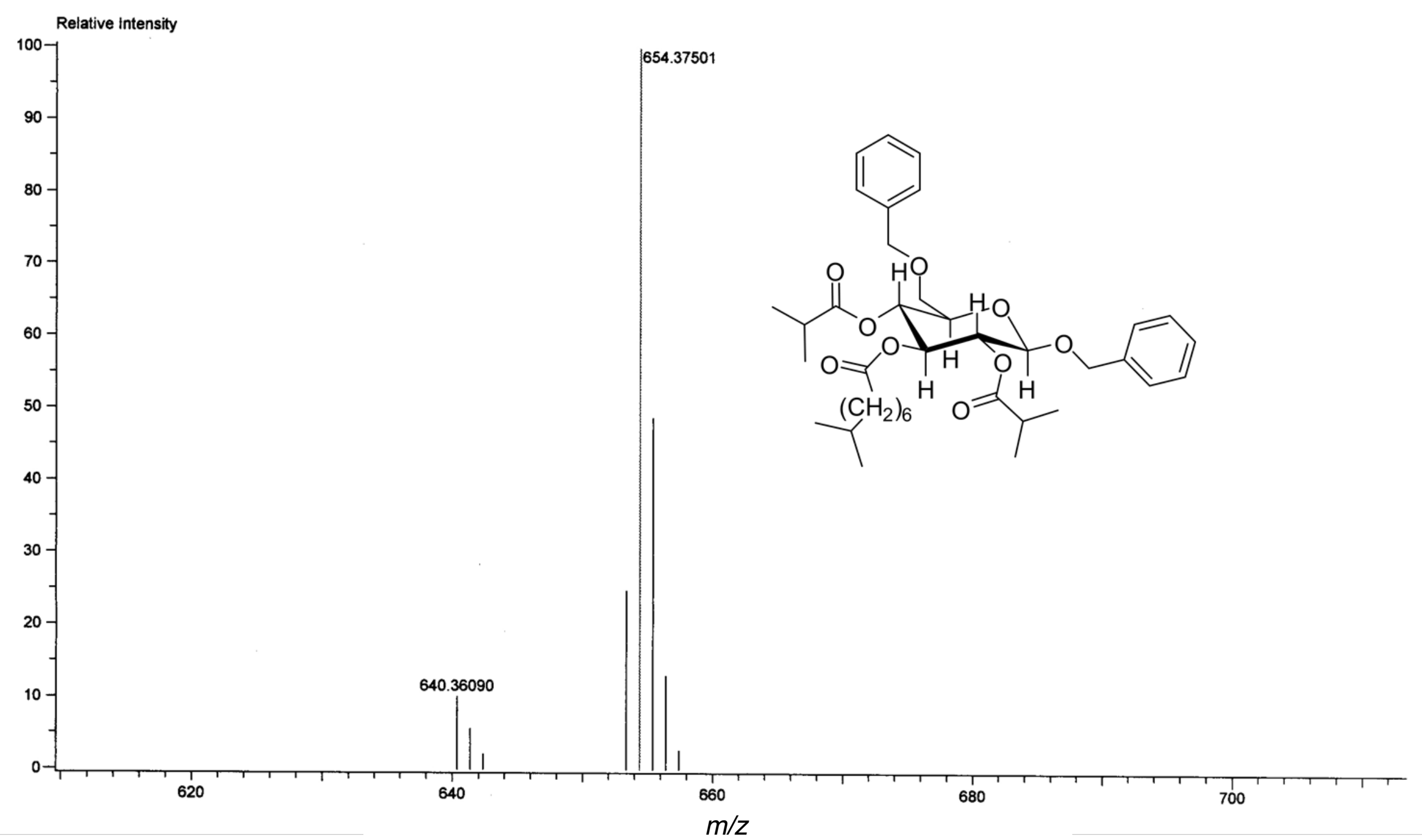

Figure S9. HRFD-MS spectrum of 5. 


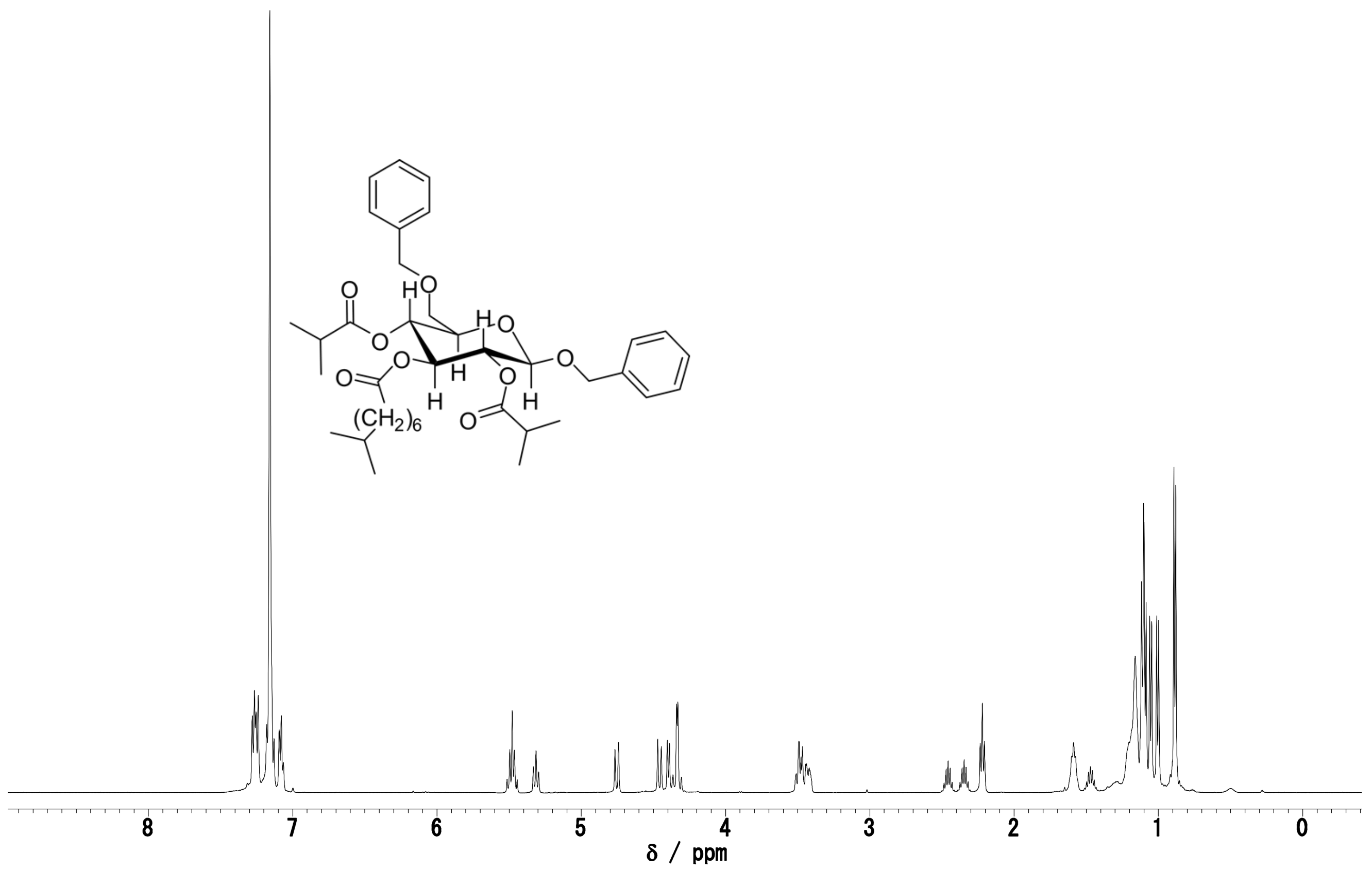

Figure S10. ${ }^{1} \mathrm{H}$ NMR spectrum of $5\left(500 \mathrm{MHz}, \mathrm{C}_{6} \mathrm{D}_{6}\right)$. 

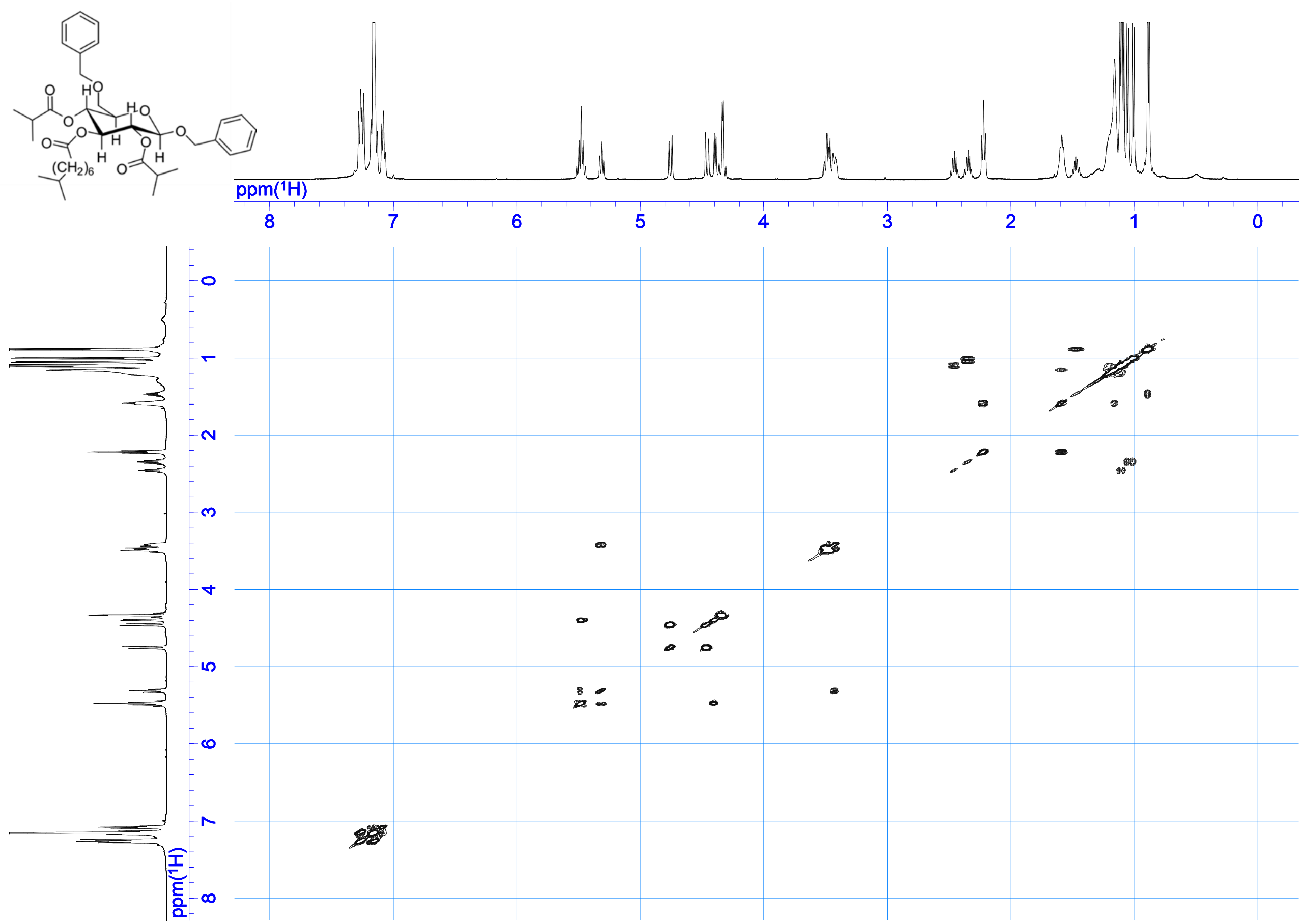

Figure S11. COSY spectrum of $5\left(500 \mathrm{MHz}, \mathrm{C}_{6} \mathrm{D}_{6}\right)$. 

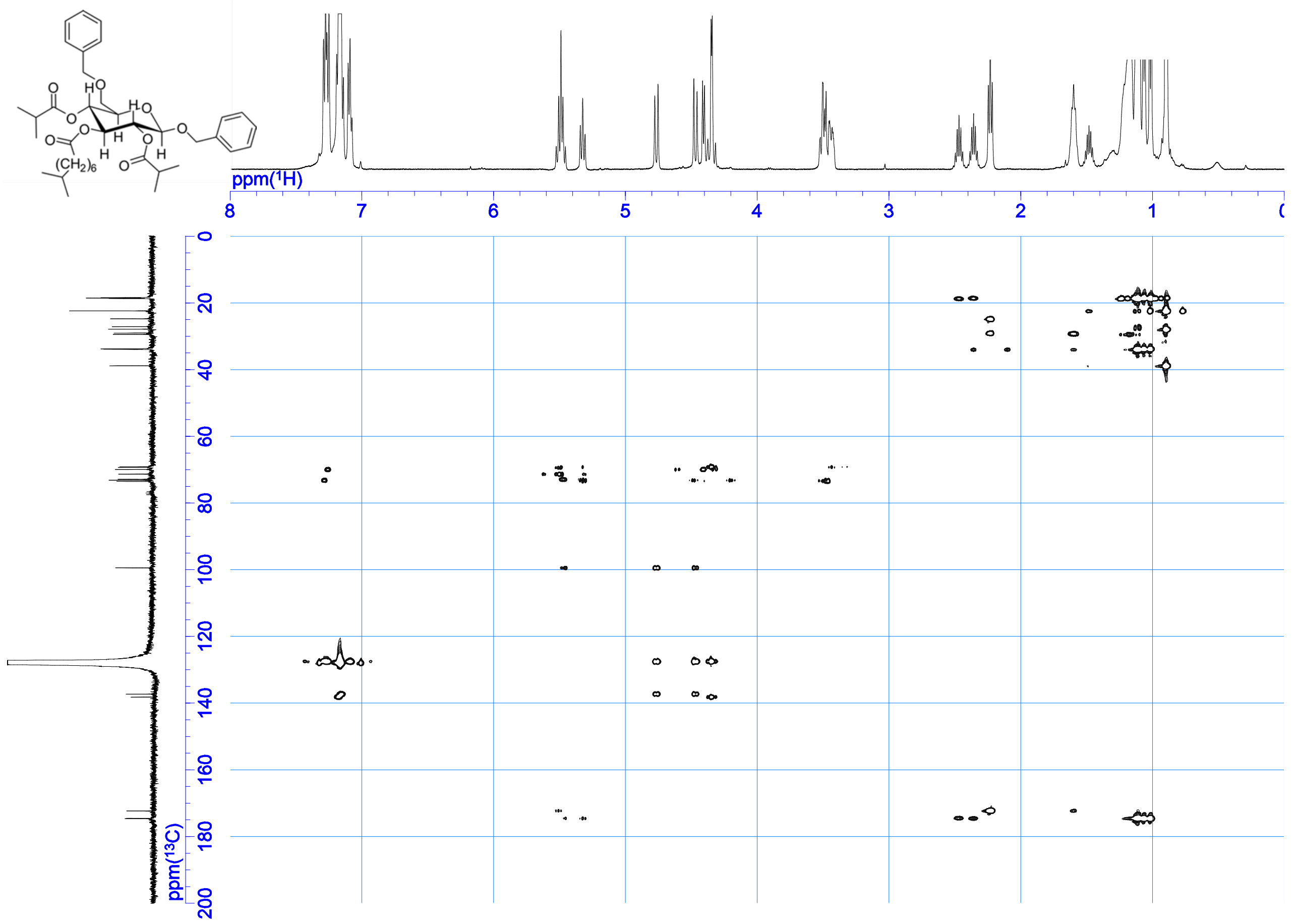

Figure S12. HMBC spectrum of $5\left(500 \mathrm{MHz}, \mathrm{C}_{6} \mathrm{D}_{6}\right)$.

19 

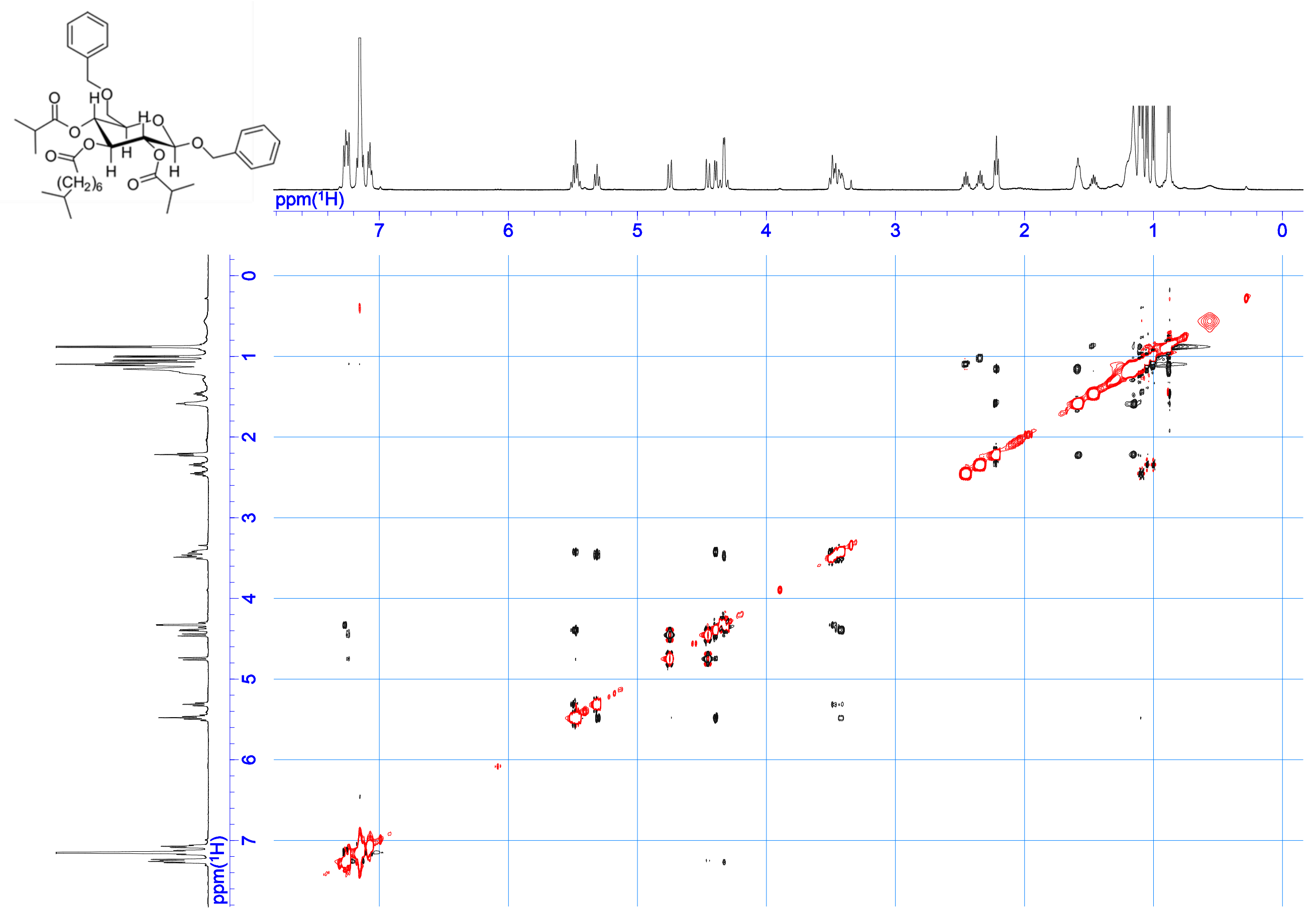

Figure S13. NOESY spectrum of $5\left(500 \mathrm{MHz}, \mathrm{C}_{6} \mathrm{D}_{6}\right)$. 


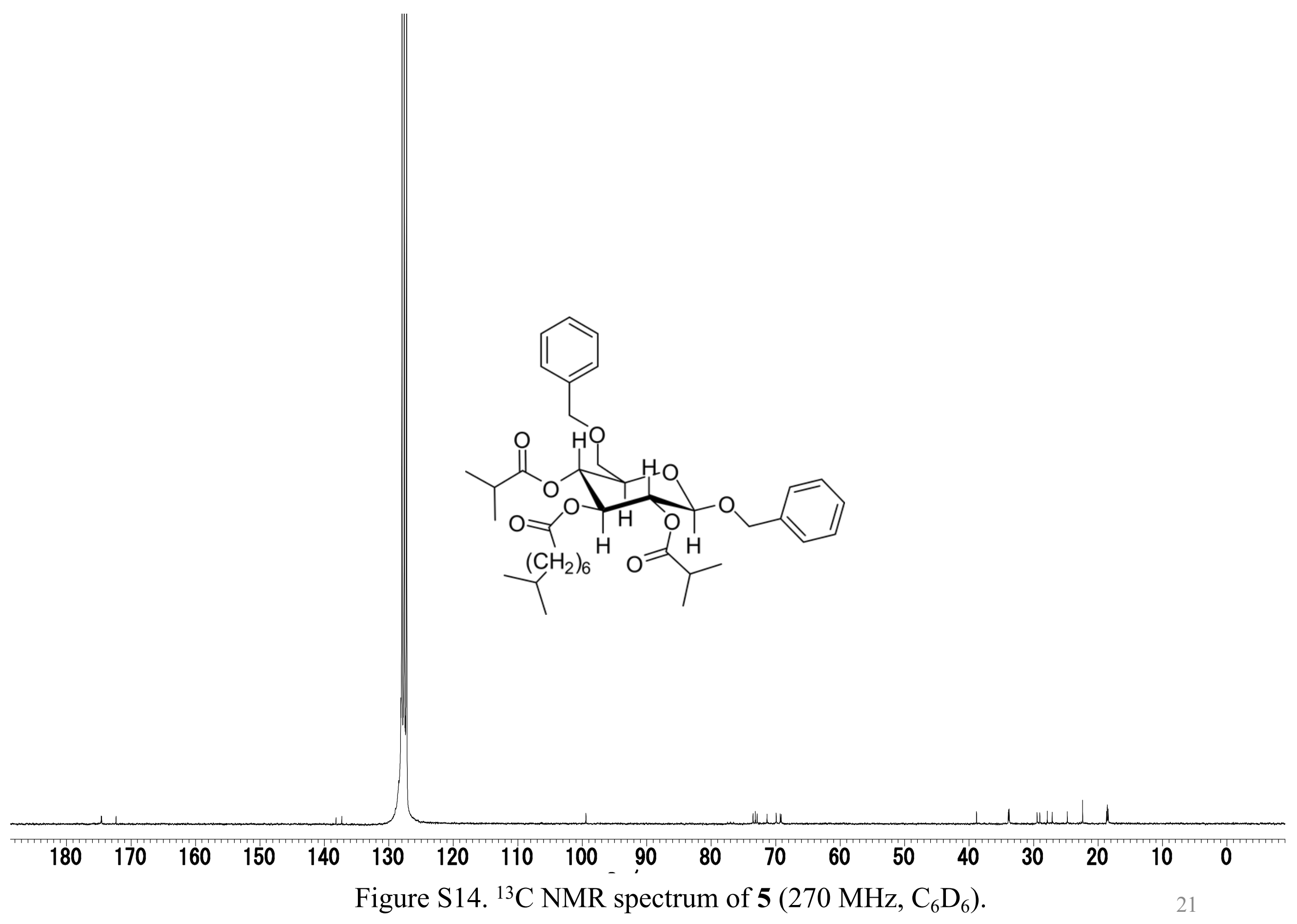



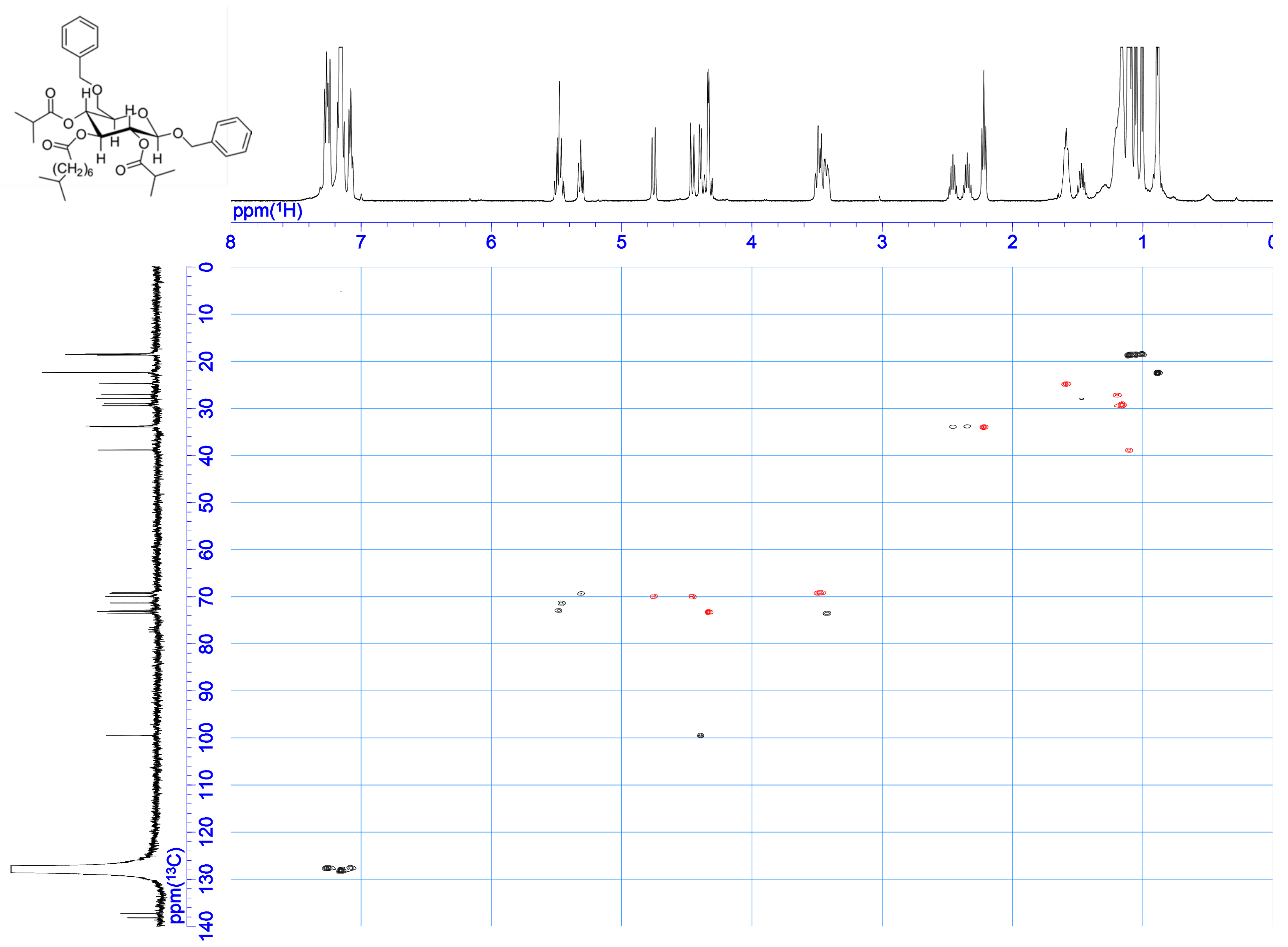

Figure S15. HMQC spectrum of $5\left(500 \mathrm{MHz}, \mathrm{C}_{6} \mathrm{D}_{6}\right)$. 


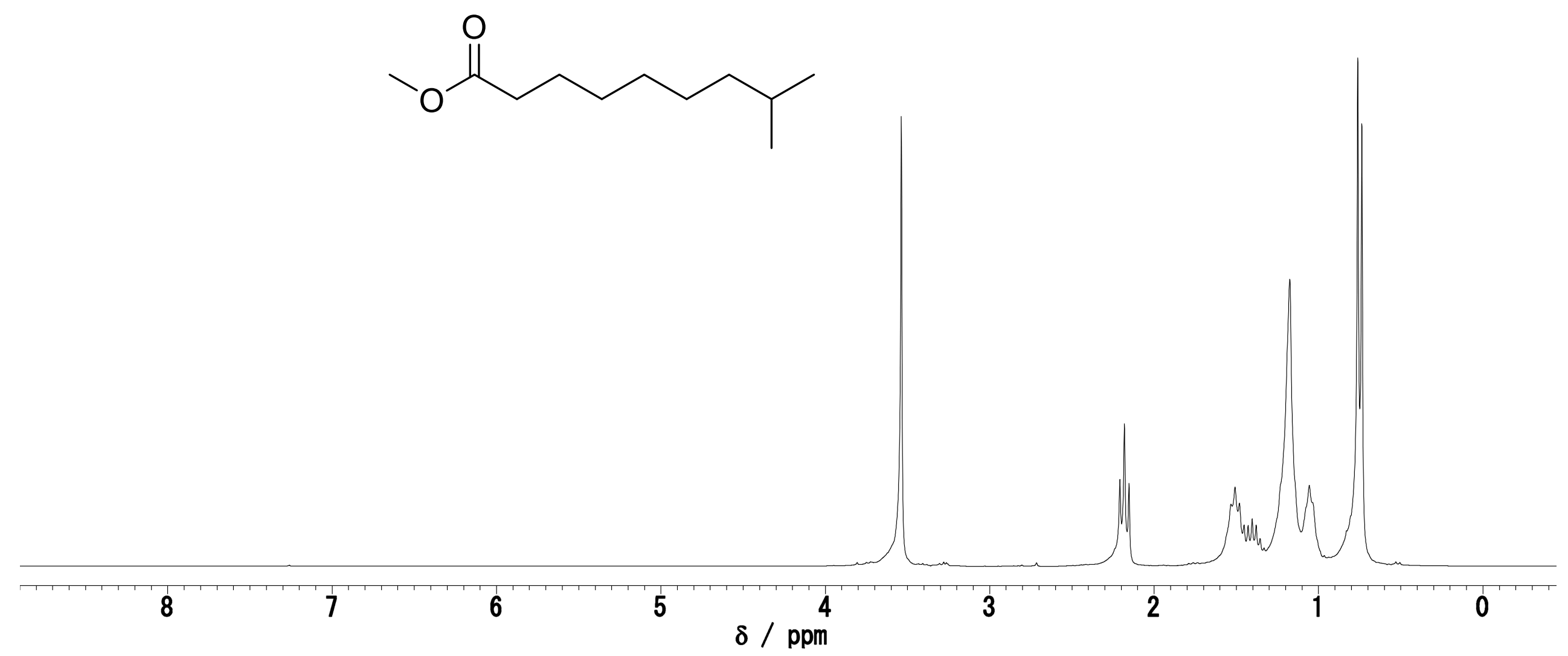

Figure S16. ${ }^{1} \mathrm{H}$ NMR spectrum of 8-methylnonanoate (7) $\left(270 \mathrm{MHz}, \mathrm{CDCl}_{3}\right)$. 


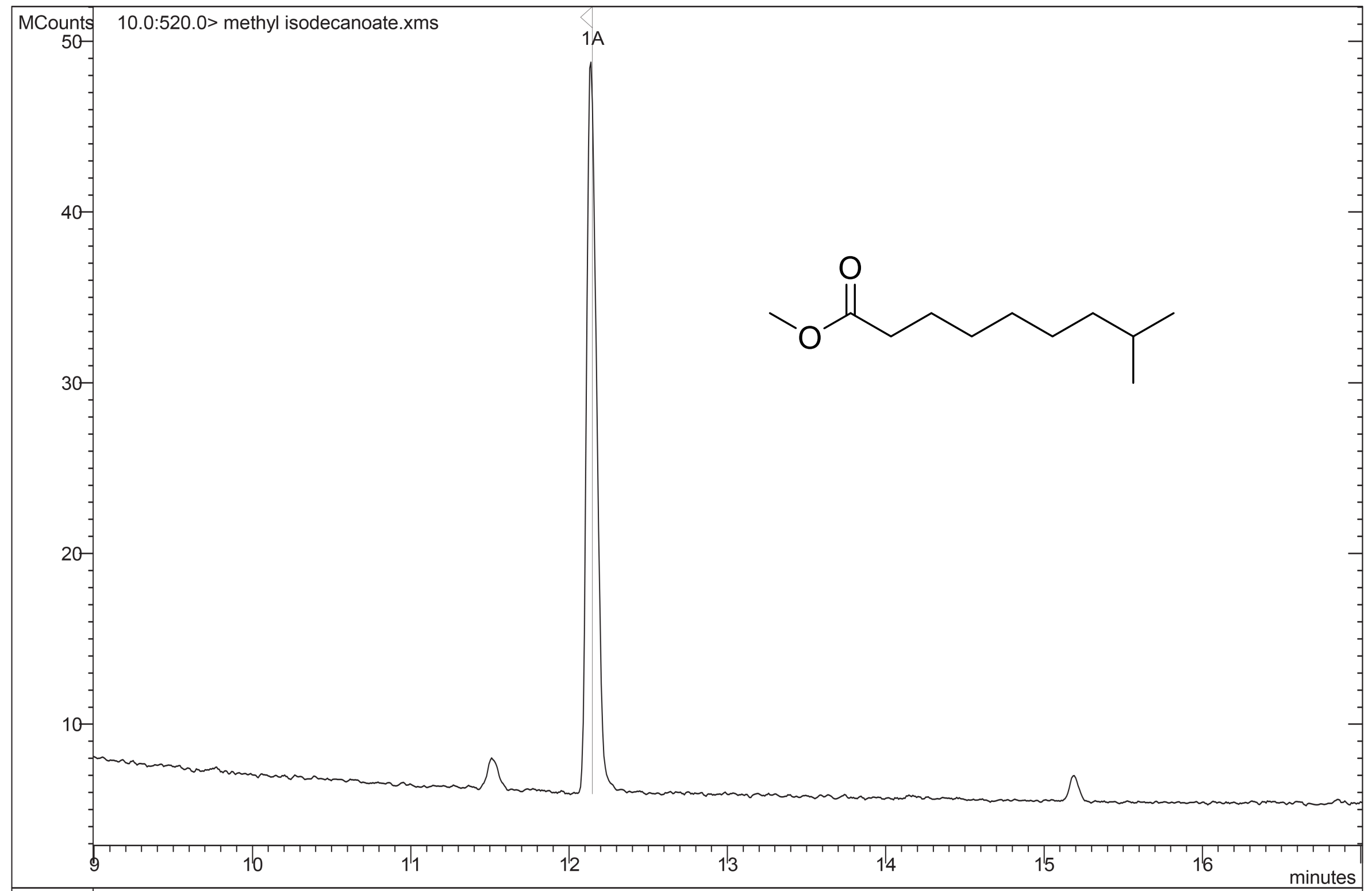

Figure S17. GC-MS chromatogram of 8-methylnonanoate (7). 


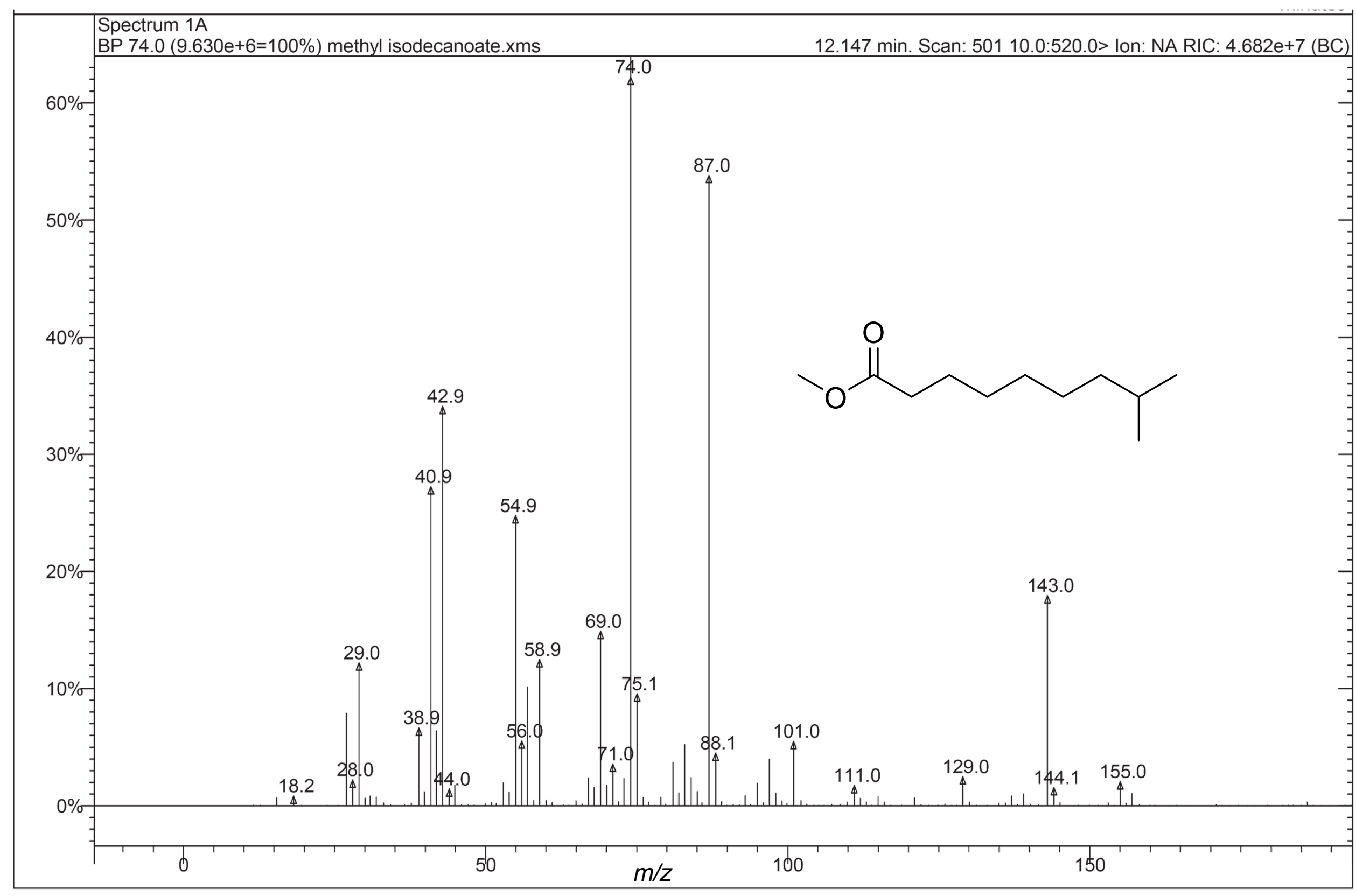

Figure S18. Fragmentation pattern of the MS peak for 8-methylnonanoate (7) having Rt. of $12.1 \mathrm{~min}$ in the chromatogram of Figure S17. 


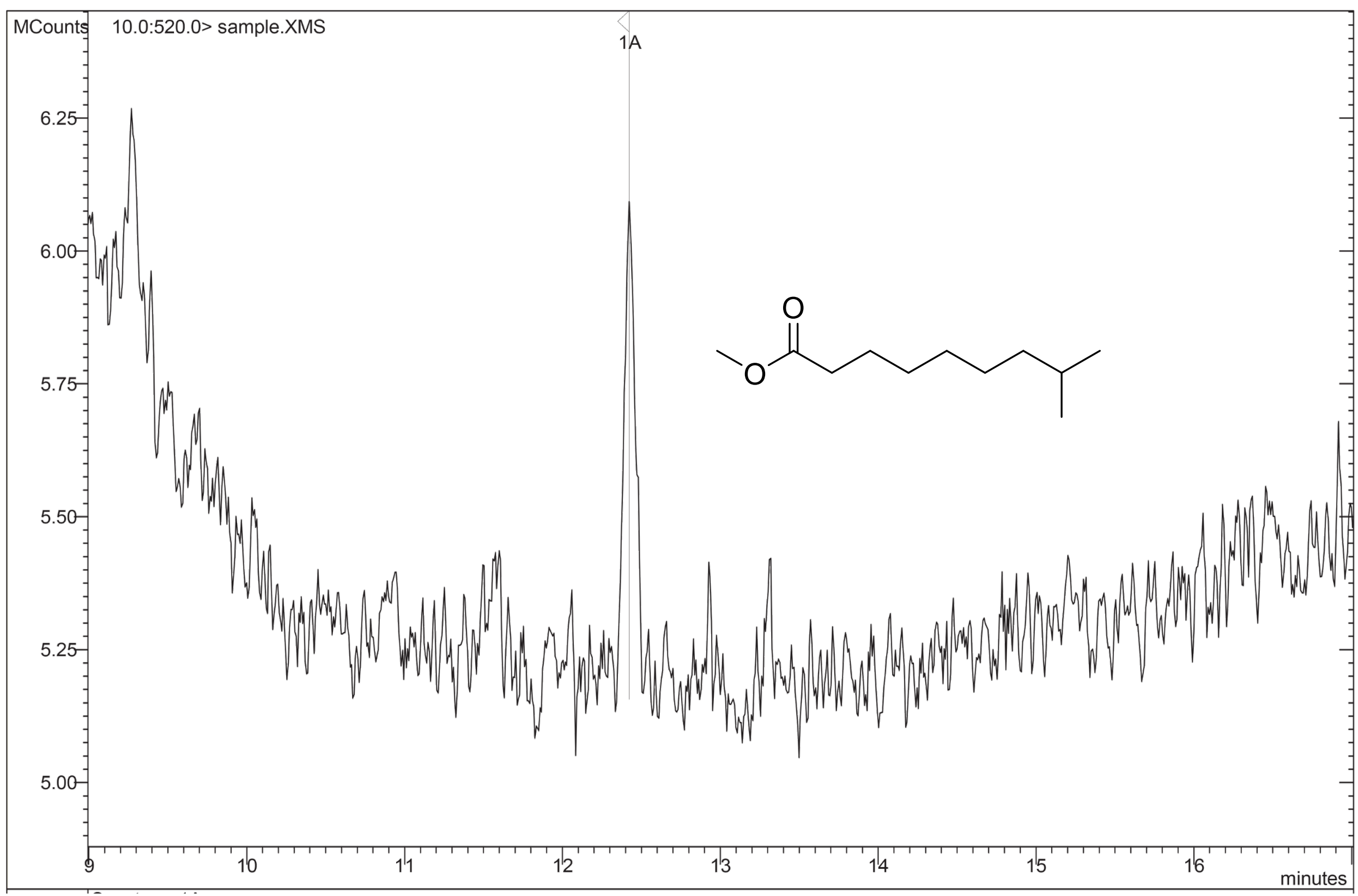

Figure S19. GC-MS chromatogram of methylated acyl moiety of $\mathbf{5}$. 


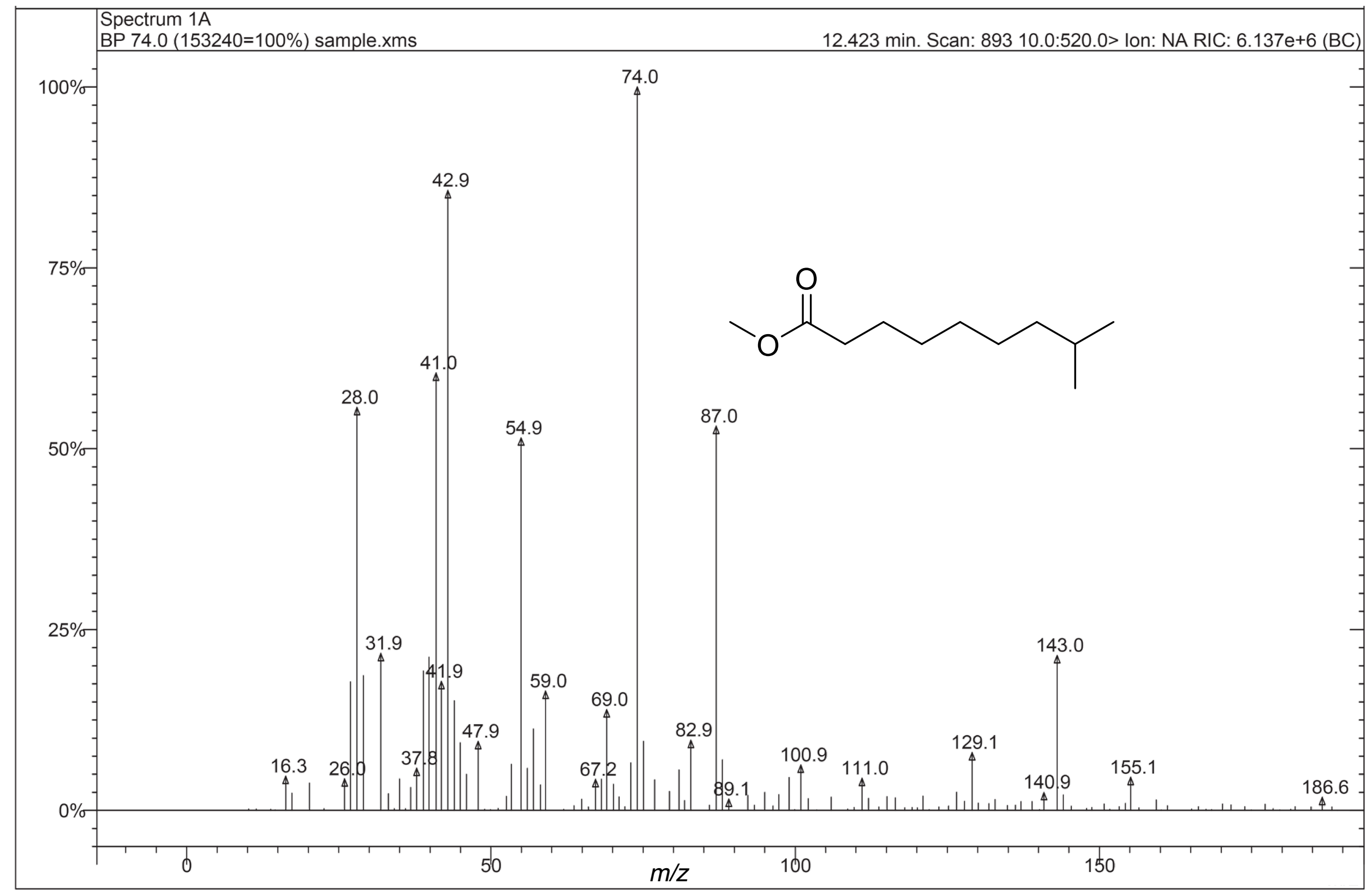

Figure S20. Fragmentation pattern of the MS peak for methylated acyl moiety of 5 having Rt. of $12.4 \mathrm{~min}$ in the chromatogram of Figure S19. 


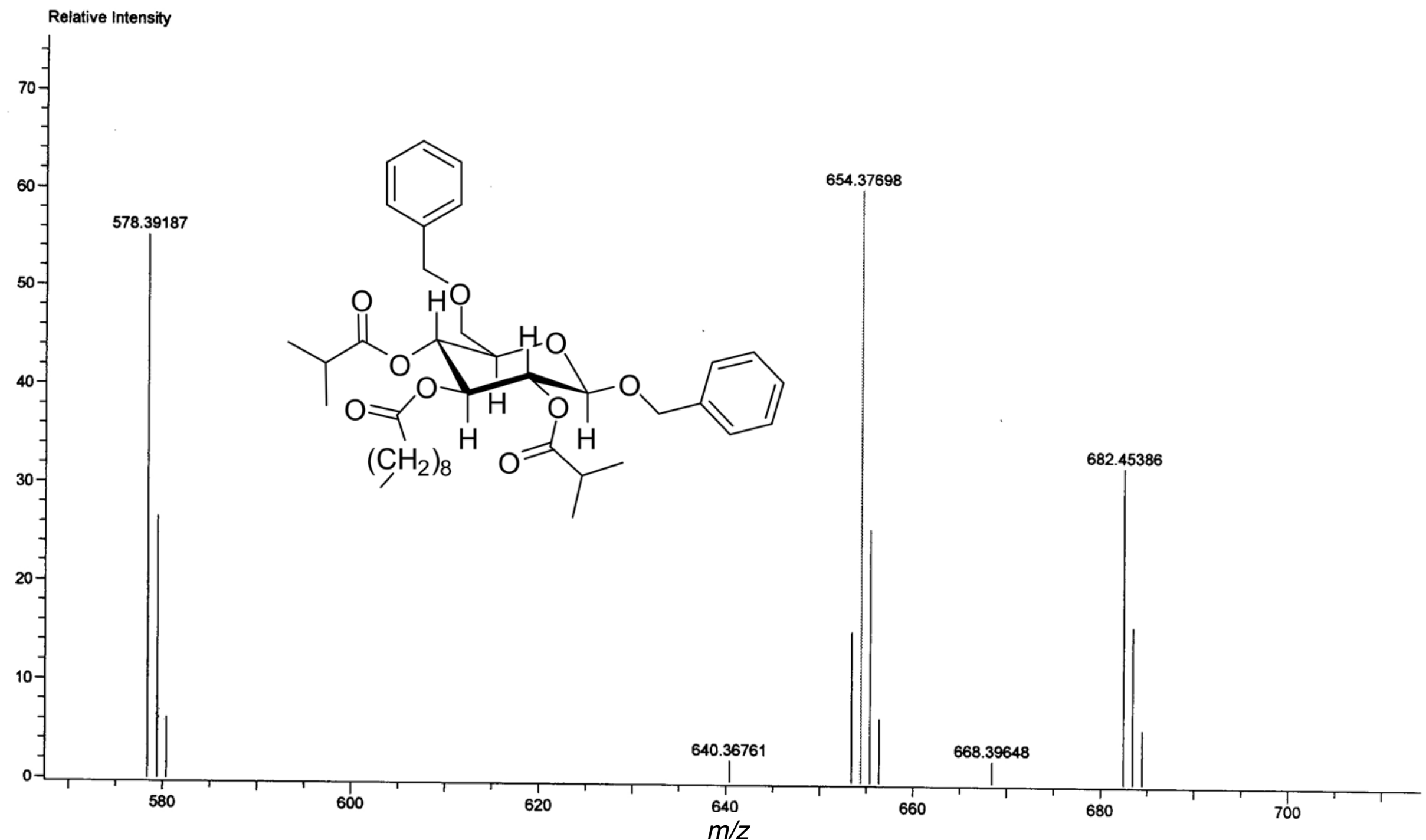

Figure S21. HRFD-MS spectrum of $\mathbf{6}$. 


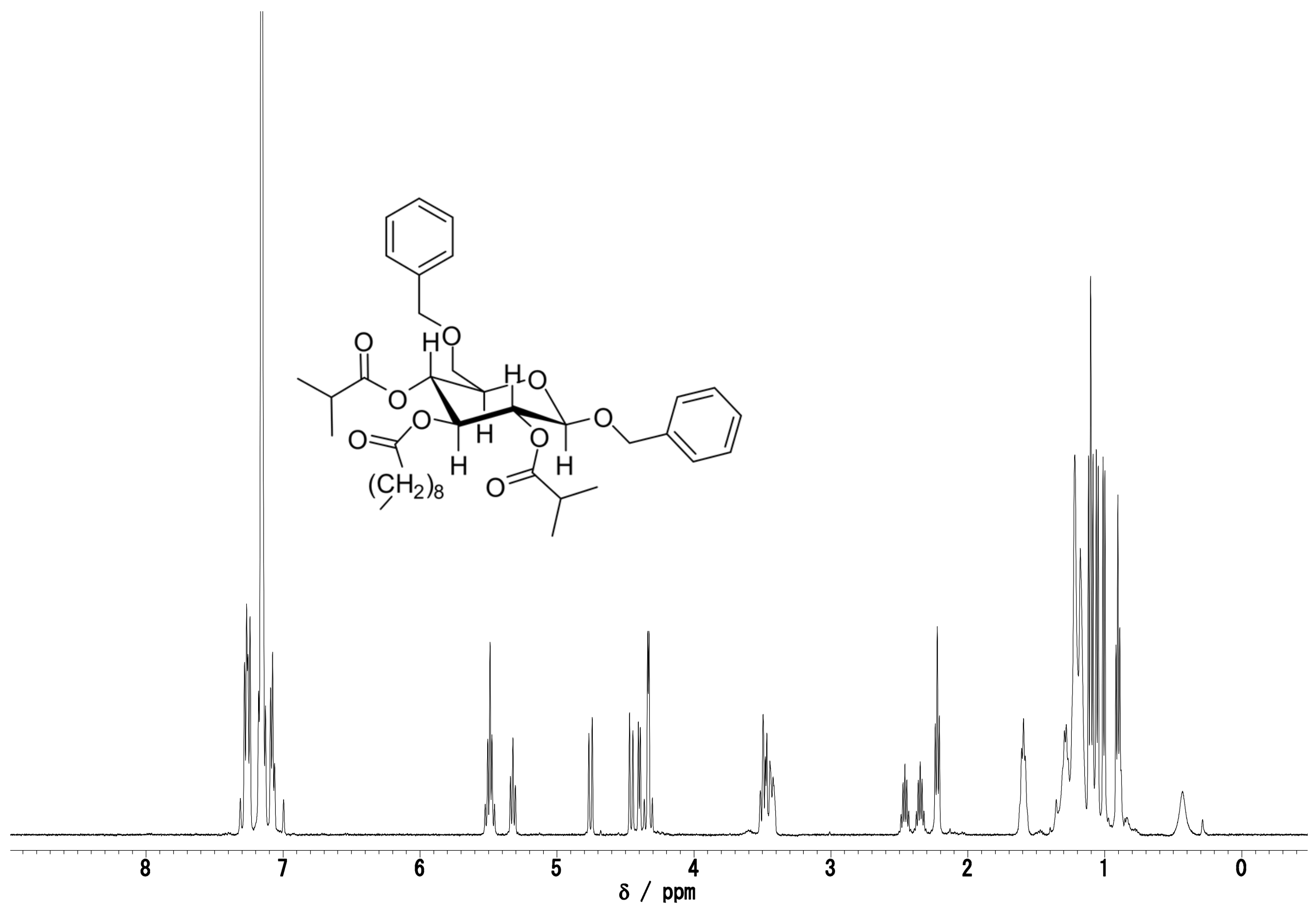

Figure S22. ${ }^{1} \mathrm{H}$ NMR spectrum of $6\left(500 \mathrm{MHz}, \mathrm{C}_{6} \mathrm{D}_{6}\right)$. 

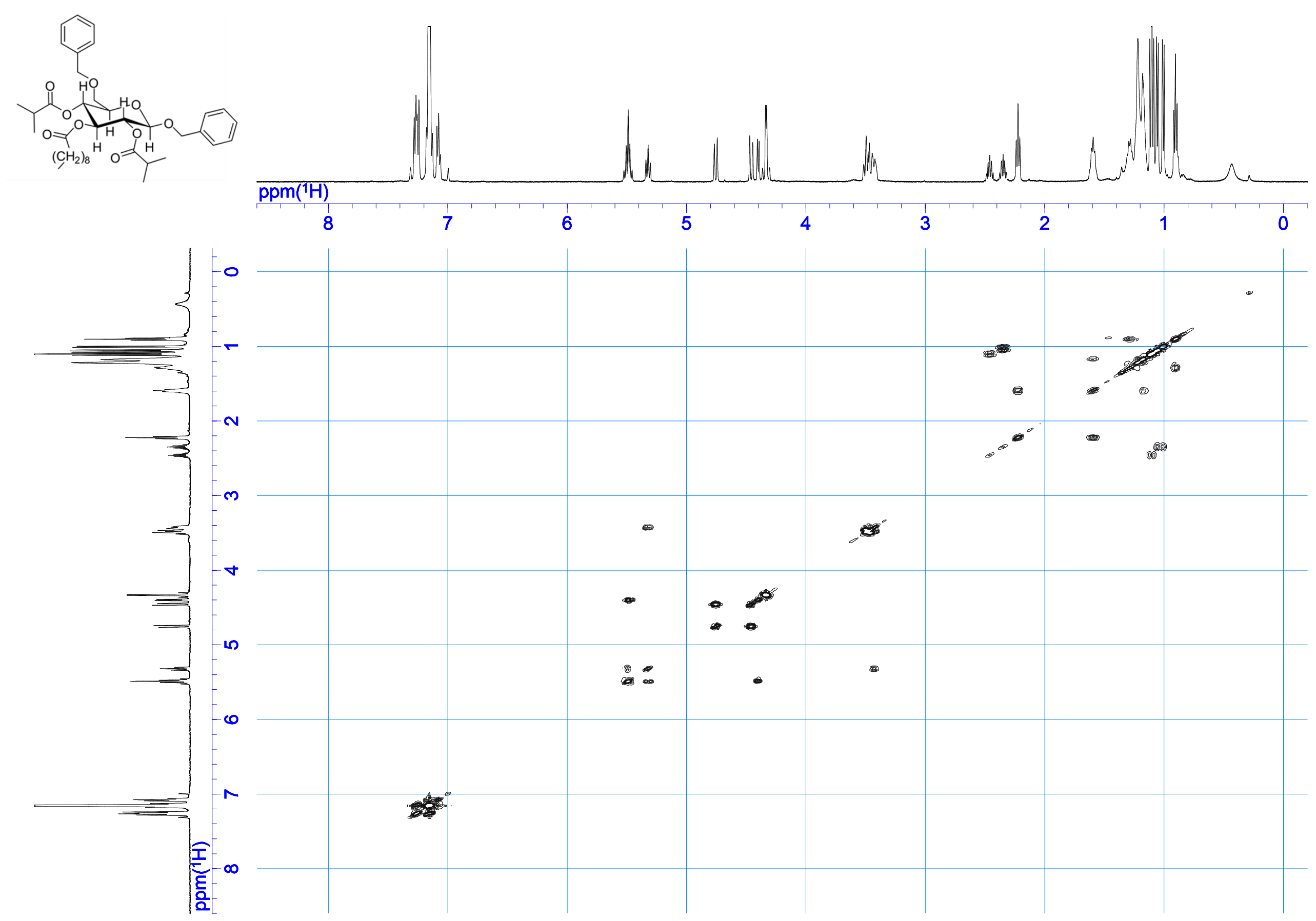

Figure S23. COSY spectrum of $6\left(500 \mathrm{MHz}, \mathrm{C}_{6} \mathrm{D}_{6}\right)$. 

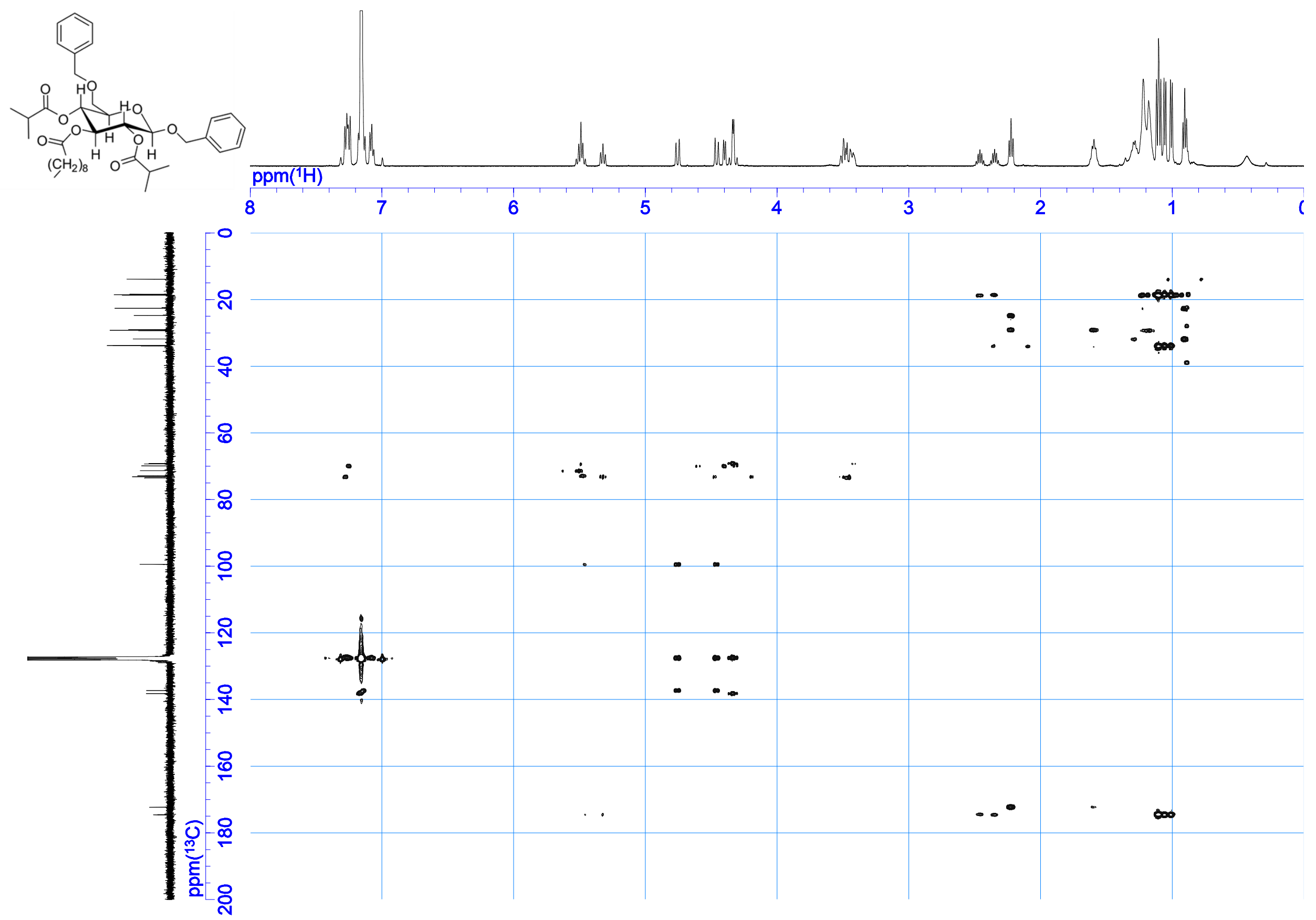

Figure S24. HMBC spectrum of $6\left(500 \mathrm{MHz}, \mathrm{C}_{6} \mathrm{D}_{6}\right)$. 

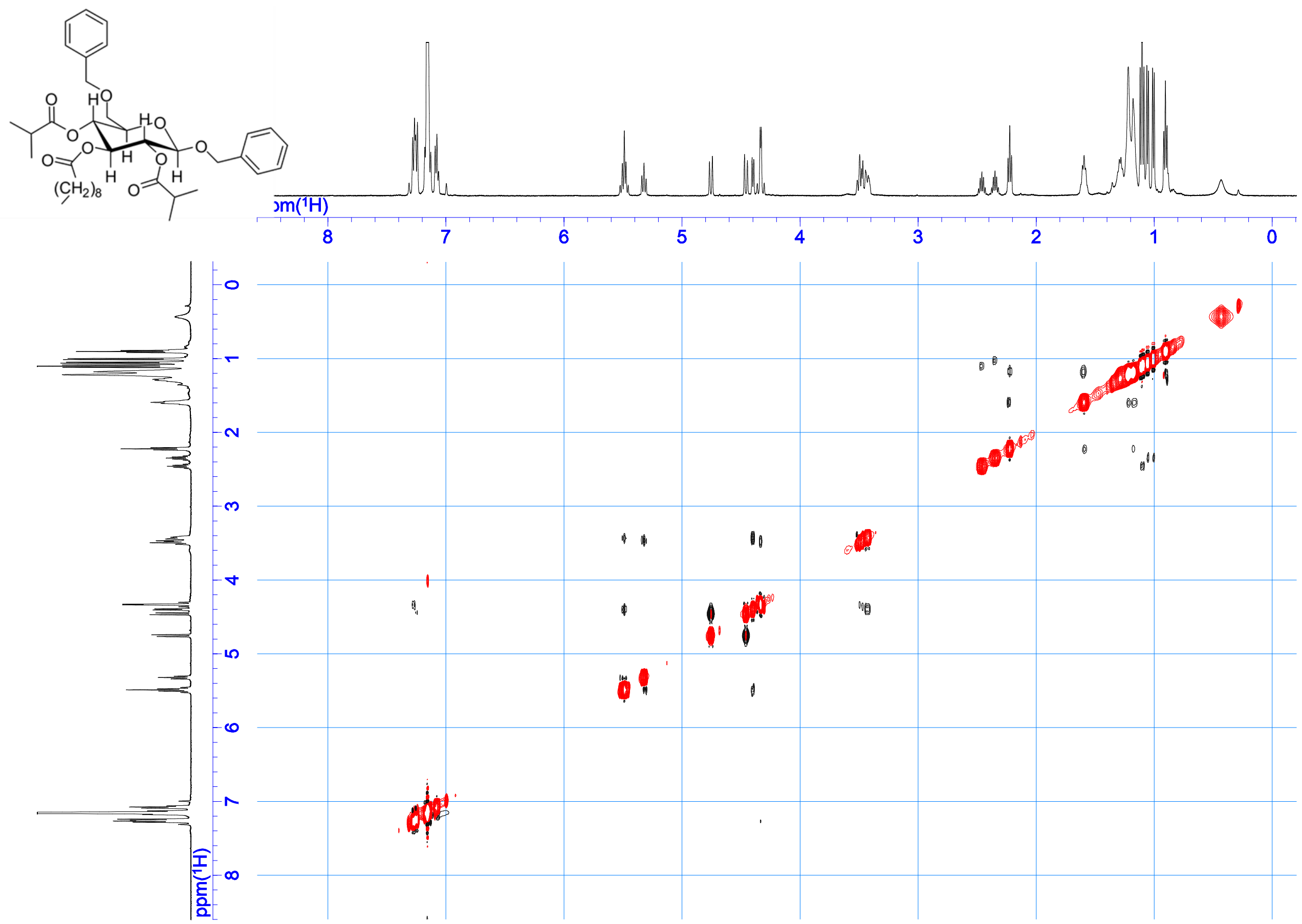

Figure S25. NOESY spectrum of $6\left(500 \mathrm{MHz}, \mathrm{C}_{6} \mathrm{D}_{6}\right)$. 


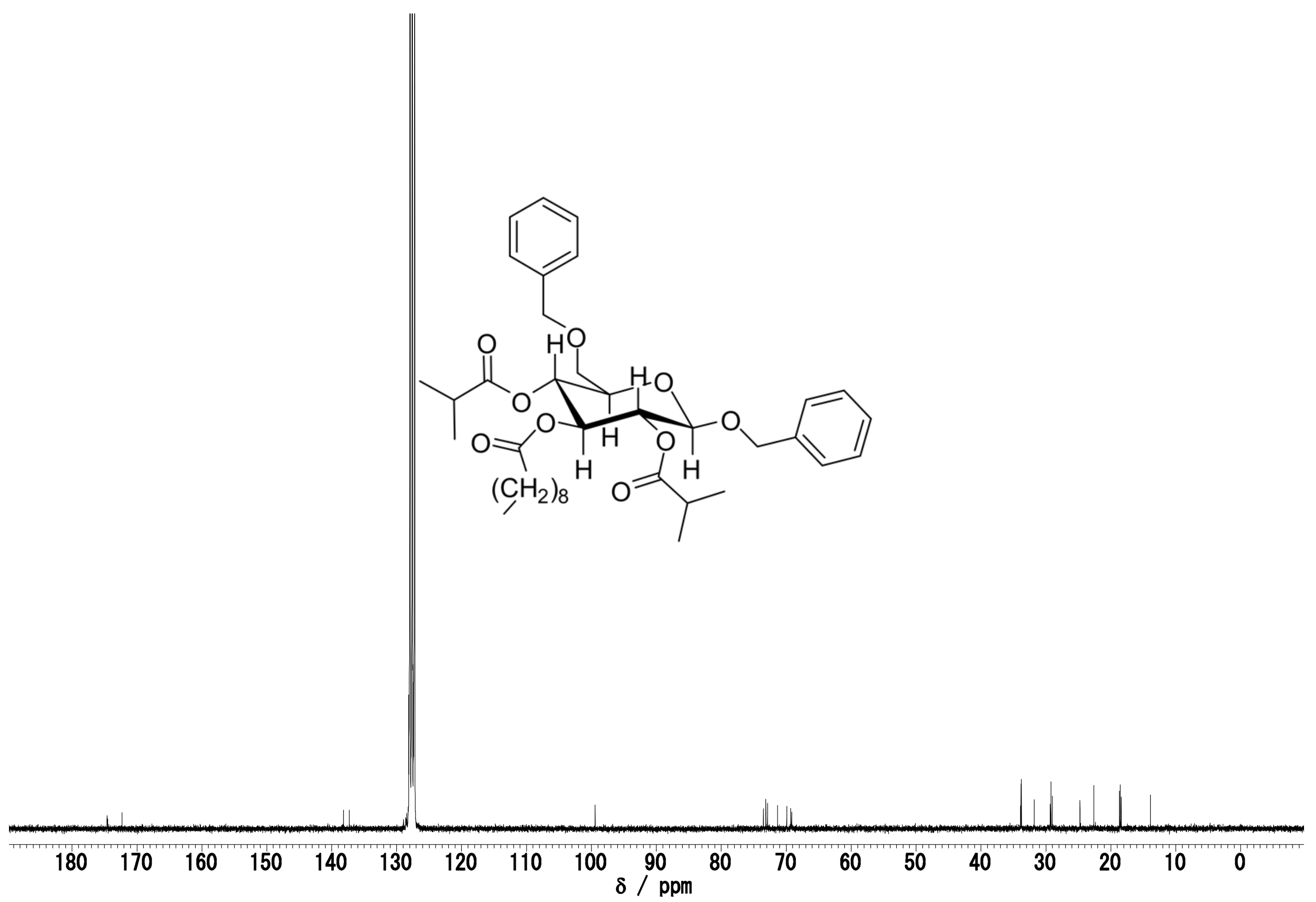

Figure S26. ${ }^{13} \mathrm{C}$ NMR spectrum of $6\left(270 \mathrm{MHz}, \mathrm{C}_{6} \mathrm{D}_{6}\right)$. 

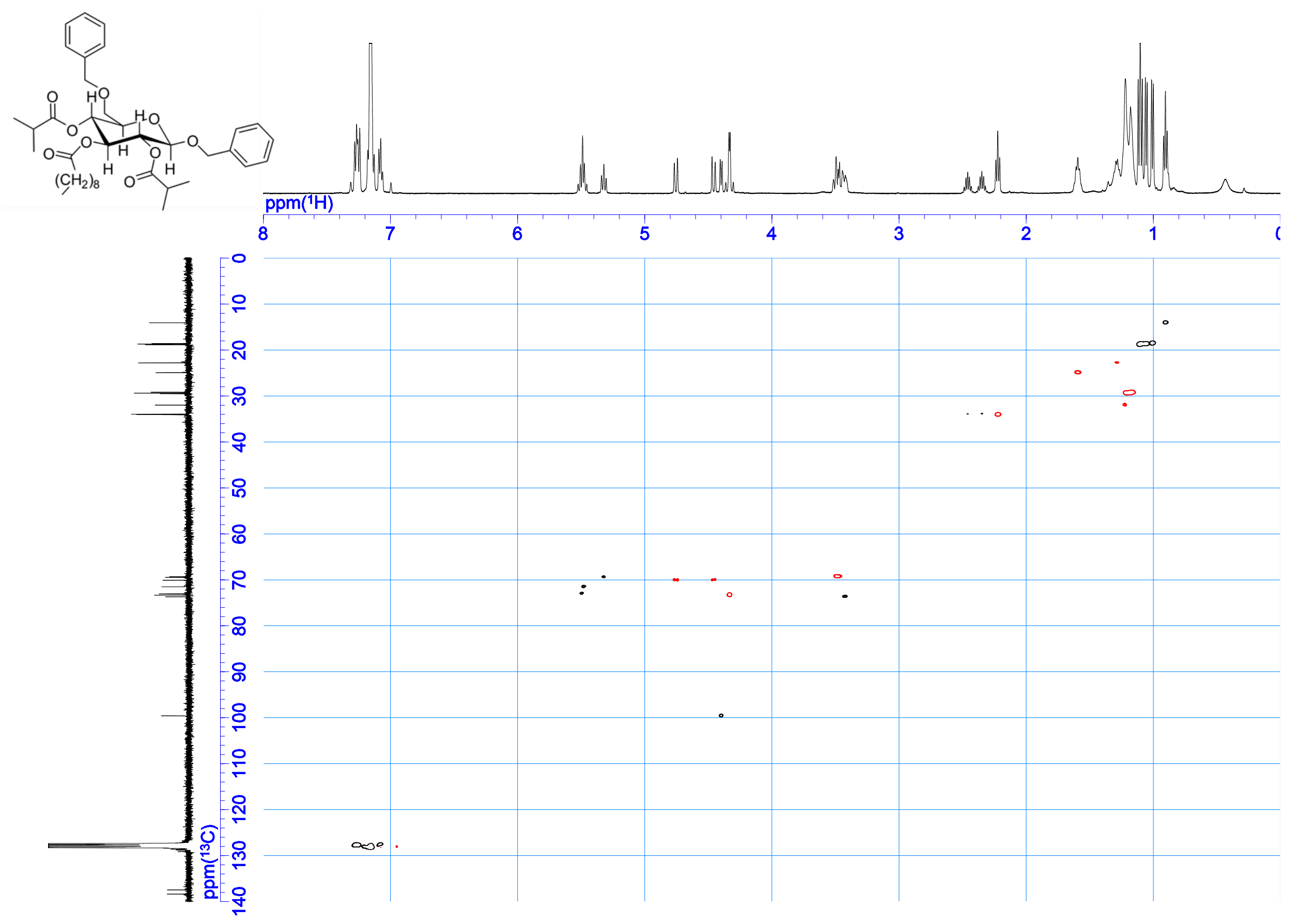

Figure S27. HMQC spectrum of $6\left(500 \mathrm{MHz}, \mathrm{C}_{6} \mathrm{D}_{6}\right)$. 


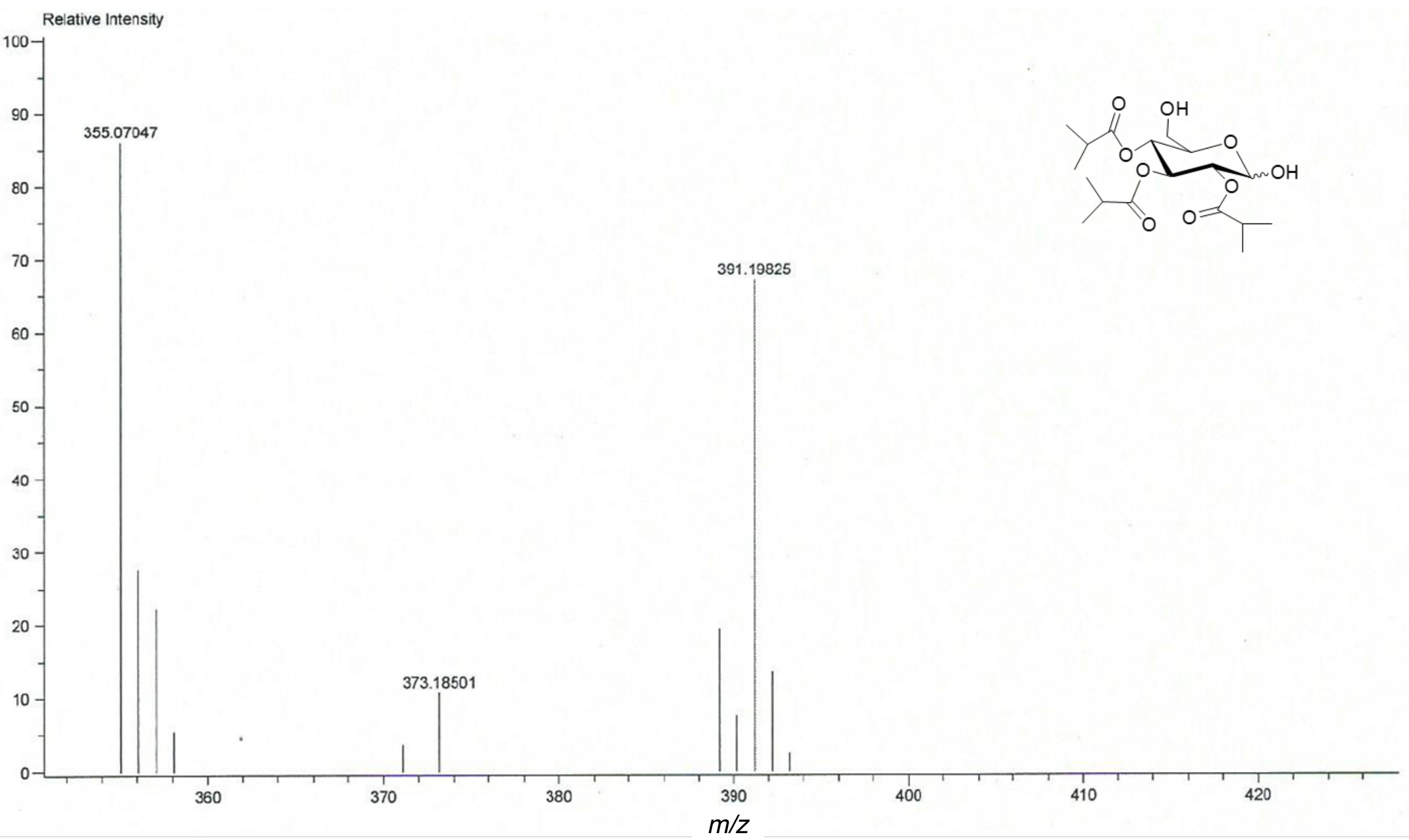

Figure S28. HRFD-MS spectrum of $\mathbf{1}$. 


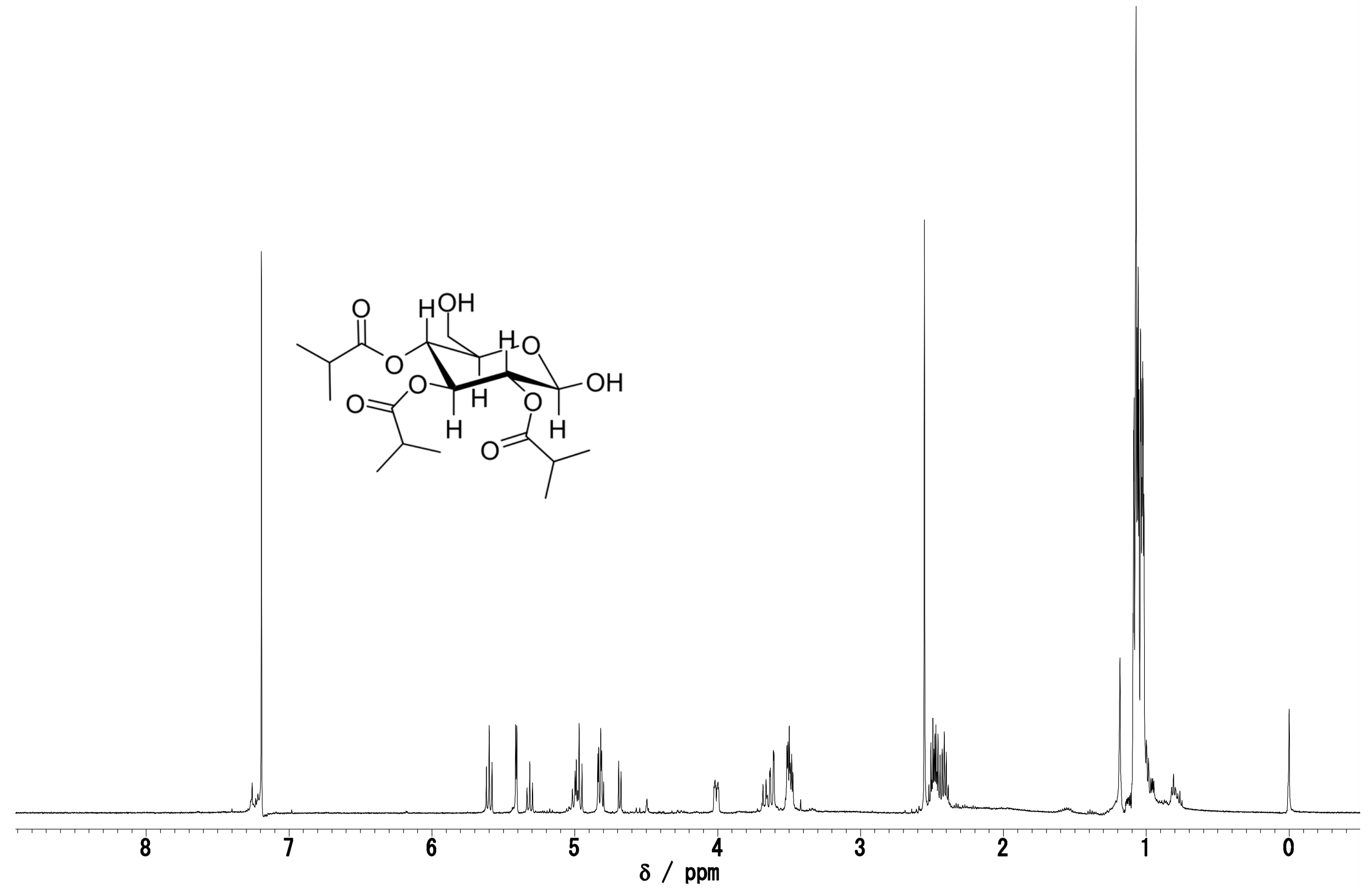

Figure S29. ${ }^{1} \mathrm{H}$ NMR spectrum of $1\left(500 \mathrm{MHz}, \mathrm{CDCl}_{3}\right)$. 


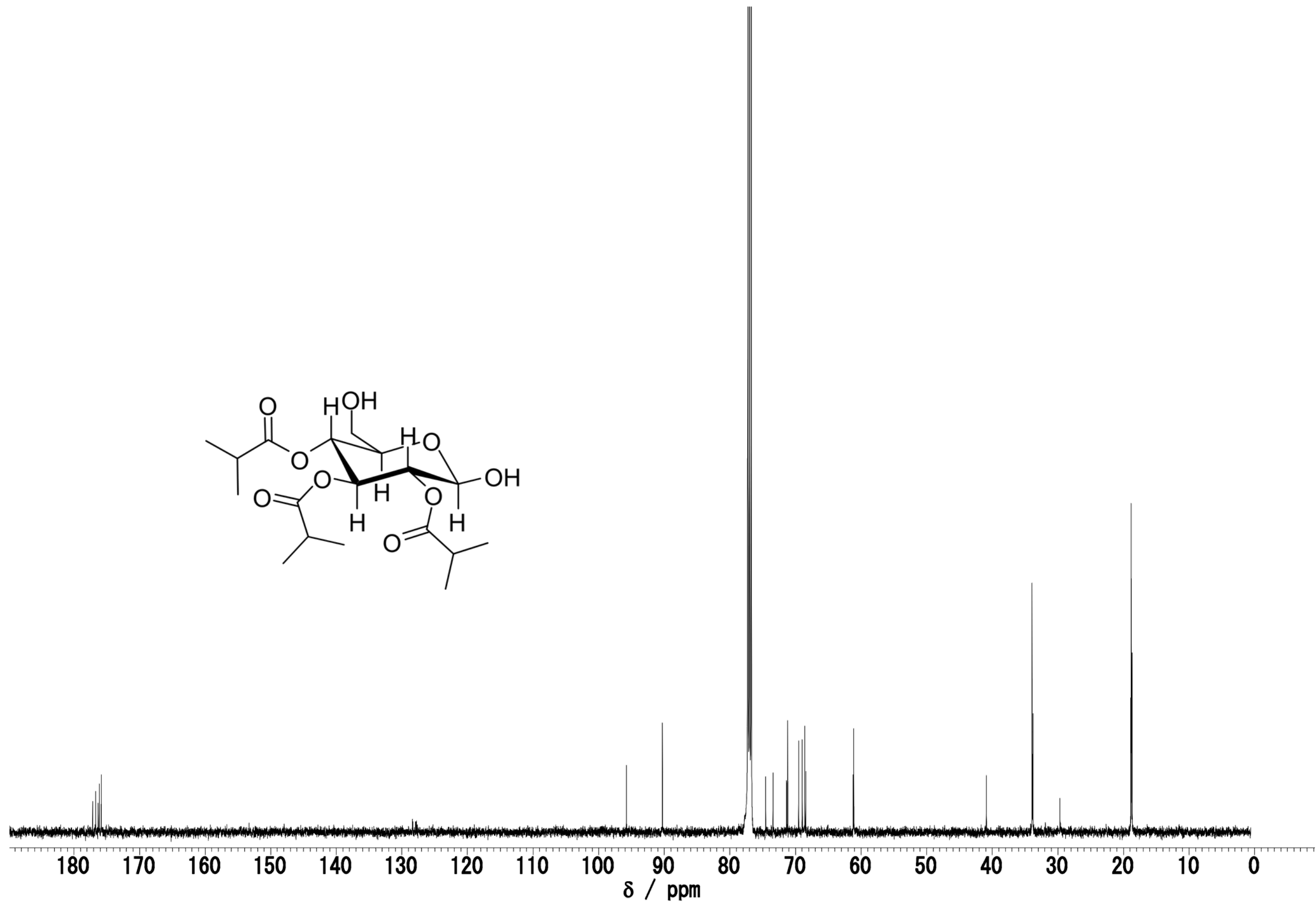

Figure S30. ${ }^{13} \mathrm{C}$ NMR spectrum of $1\left(500 \mathrm{MHz}, \mathrm{CDCl}_{3}\right)$. 


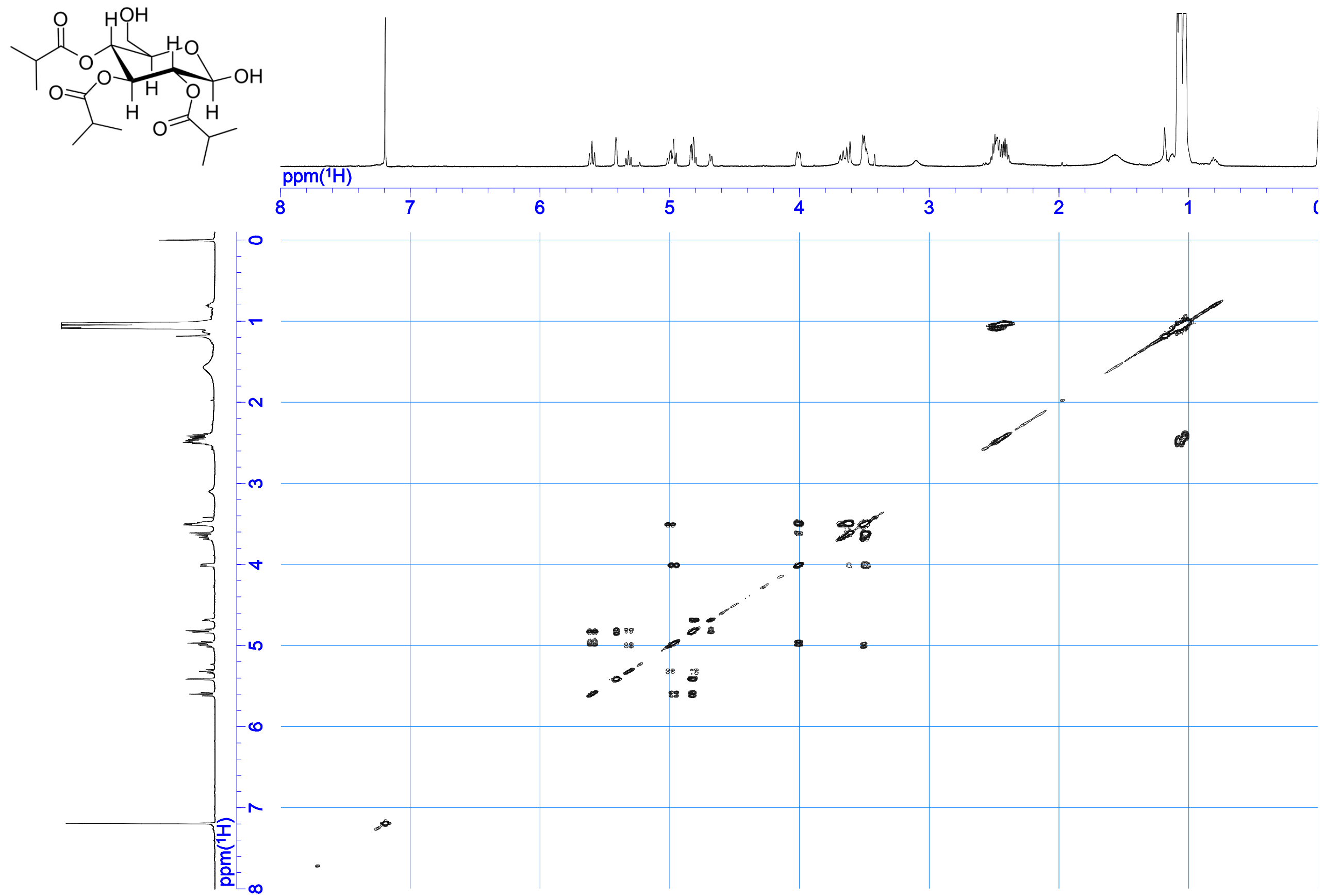

Figure S31. COSY spectrum of $1\left(500 \mathrm{MHz}, \mathrm{CDCl}_{3}\right)$. 

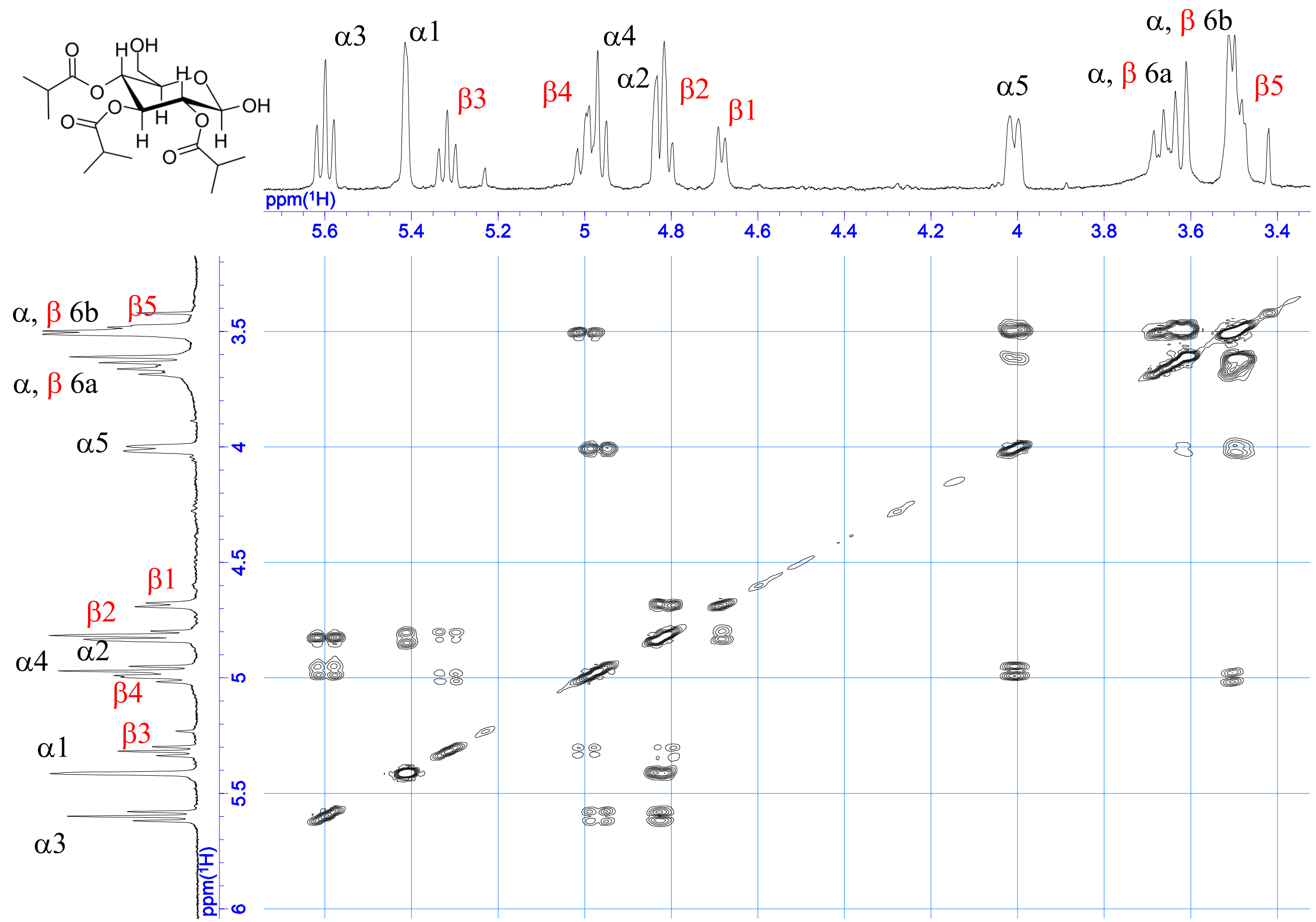

Figure S32. COSY spectrum of $1\left(500 \mathrm{MHz}, \mathrm{CDCl}_{3}\right)$. 


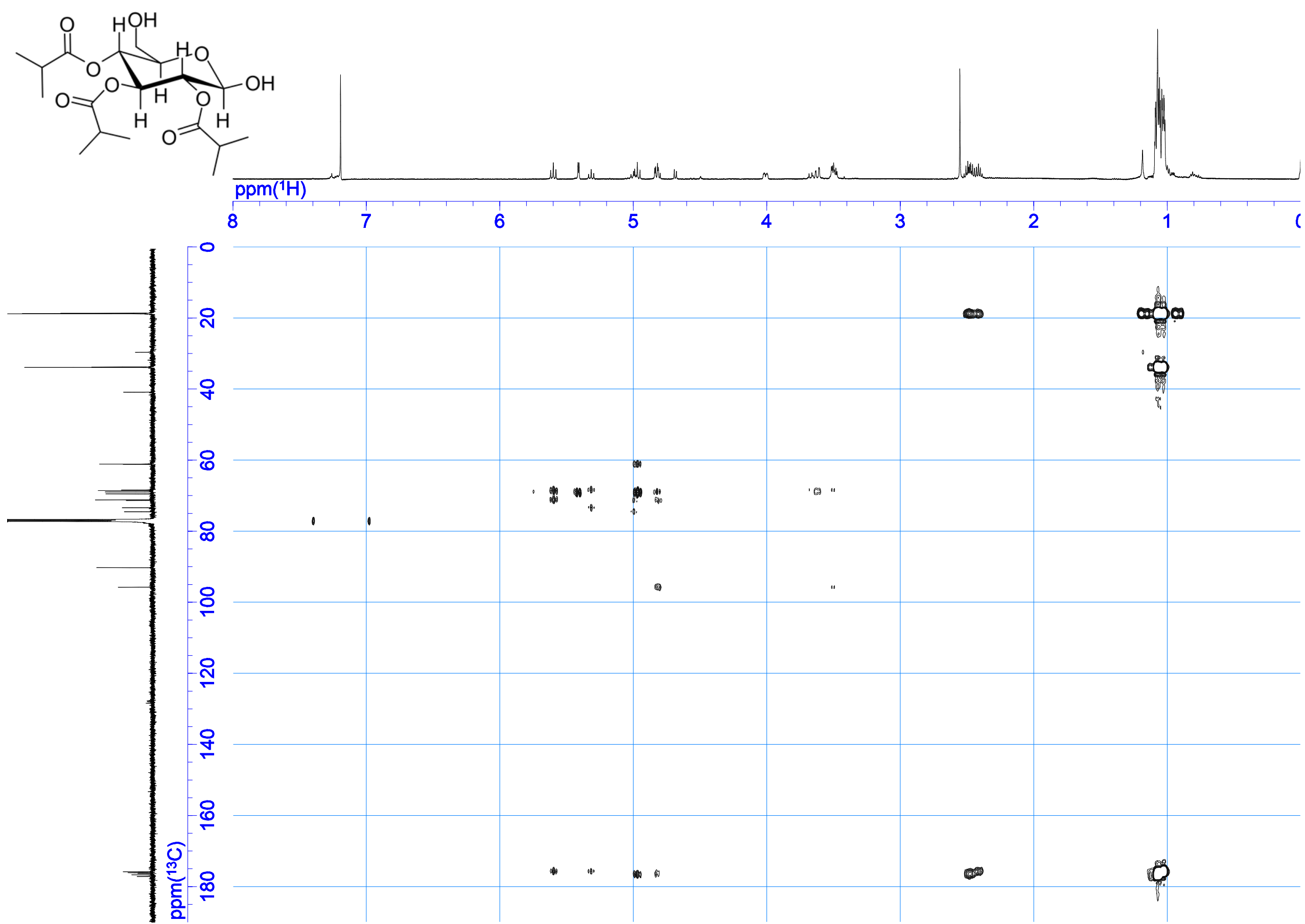

Figure S33. HMBC spectrum of $1\left(500 \mathrm{MHz}, \mathrm{CDCl}_{3}\right)$. 

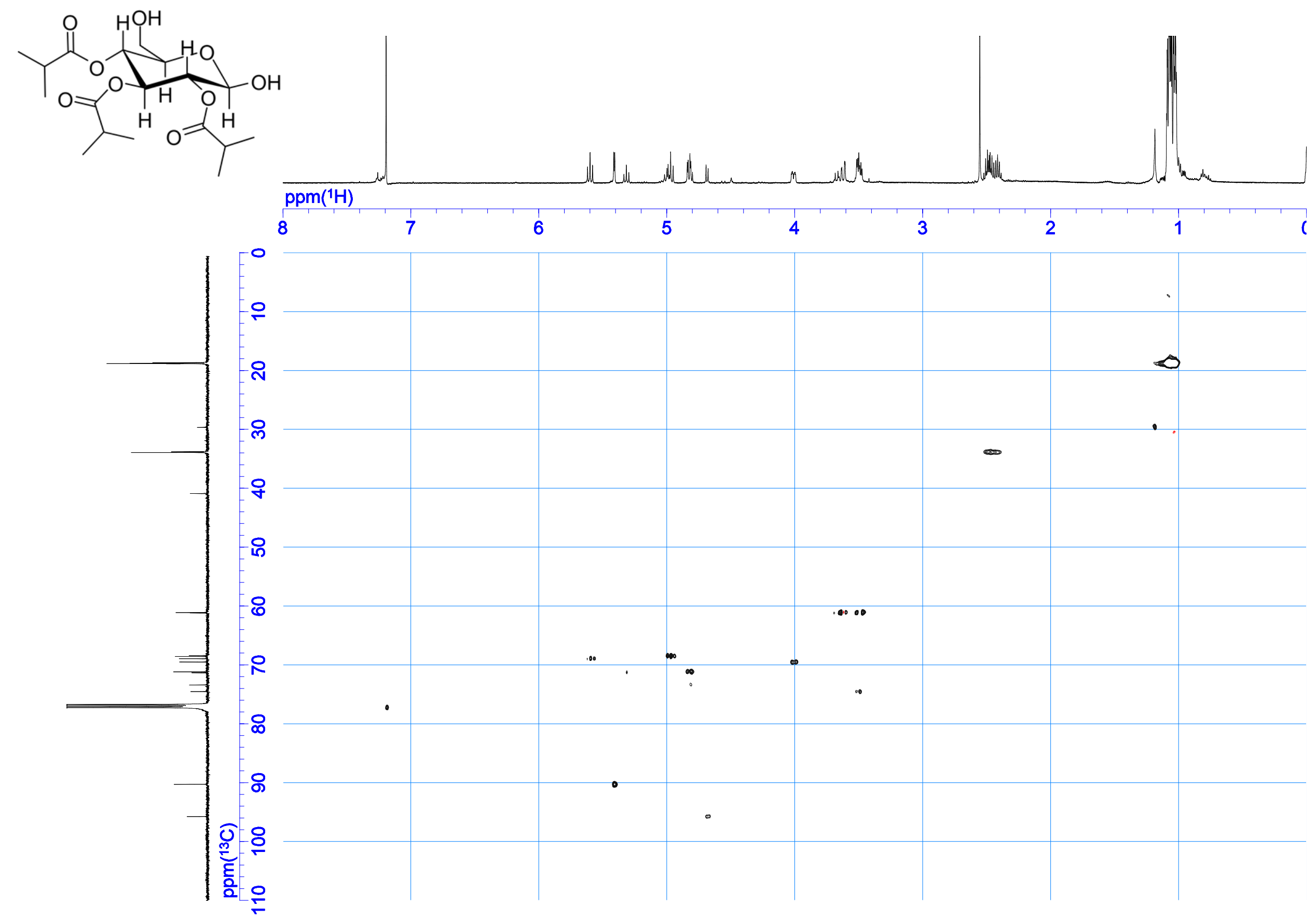

Figure S34. HMQC spectrum of $1\left(500 \mathrm{MHz}, \mathrm{CDCl}_{3}\right)$. 


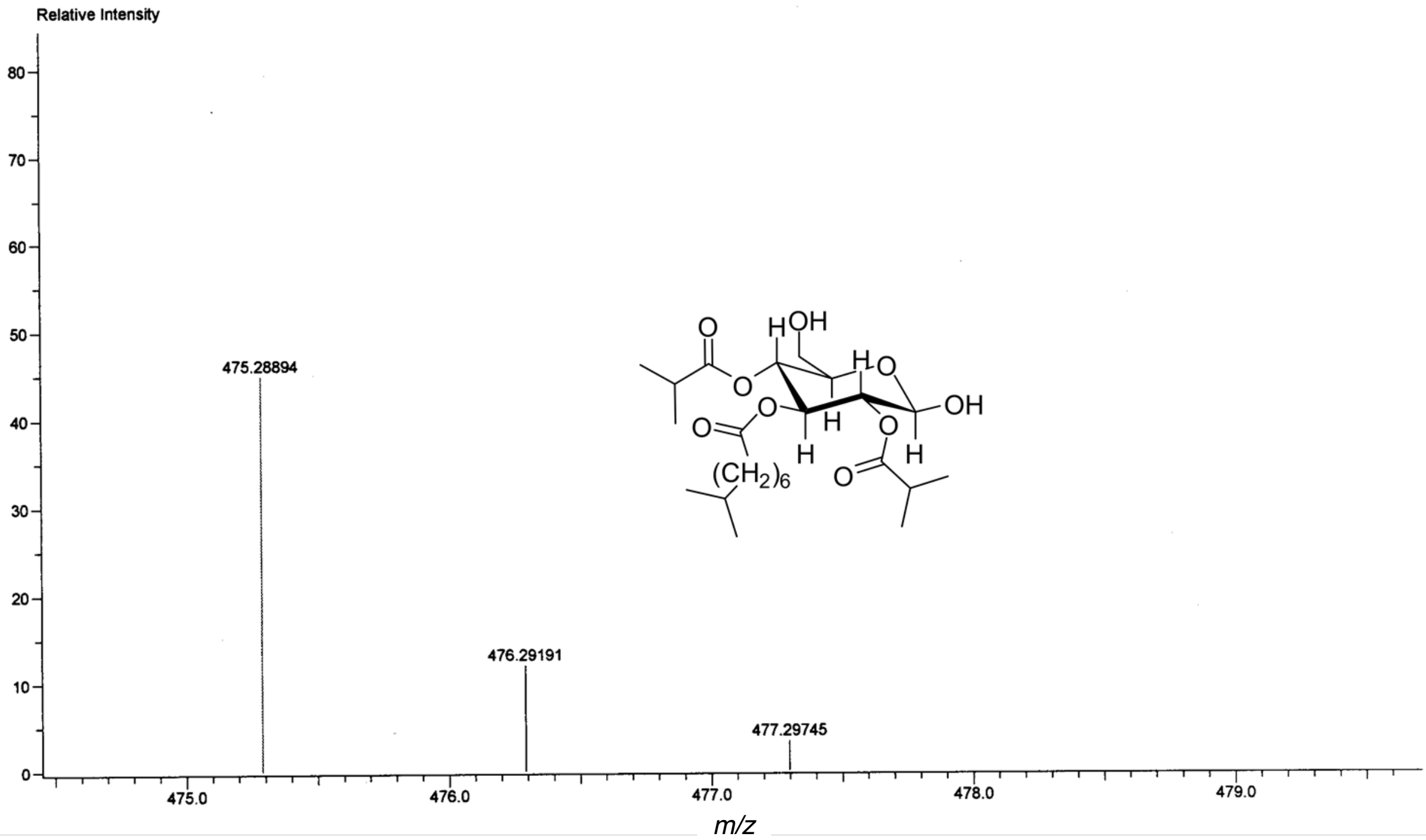

Figure S35. HRFD-MS spectrum of 2. 


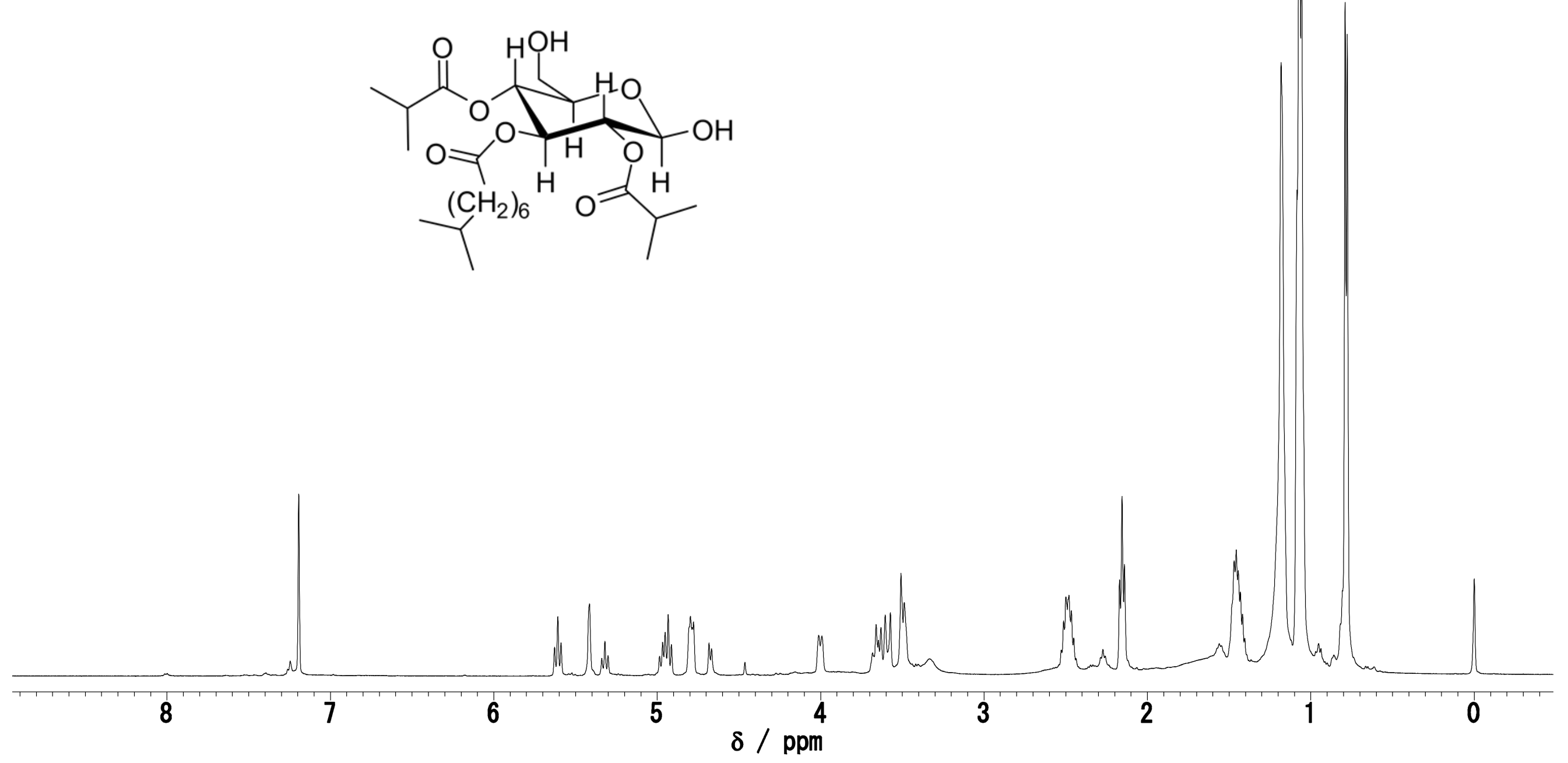

Figure S36. ${ }^{1} \mathrm{H}$ NMR spectrum of $2\left(500 \mathrm{MHz}, \mathrm{CDCl}_{3}\right)$. 

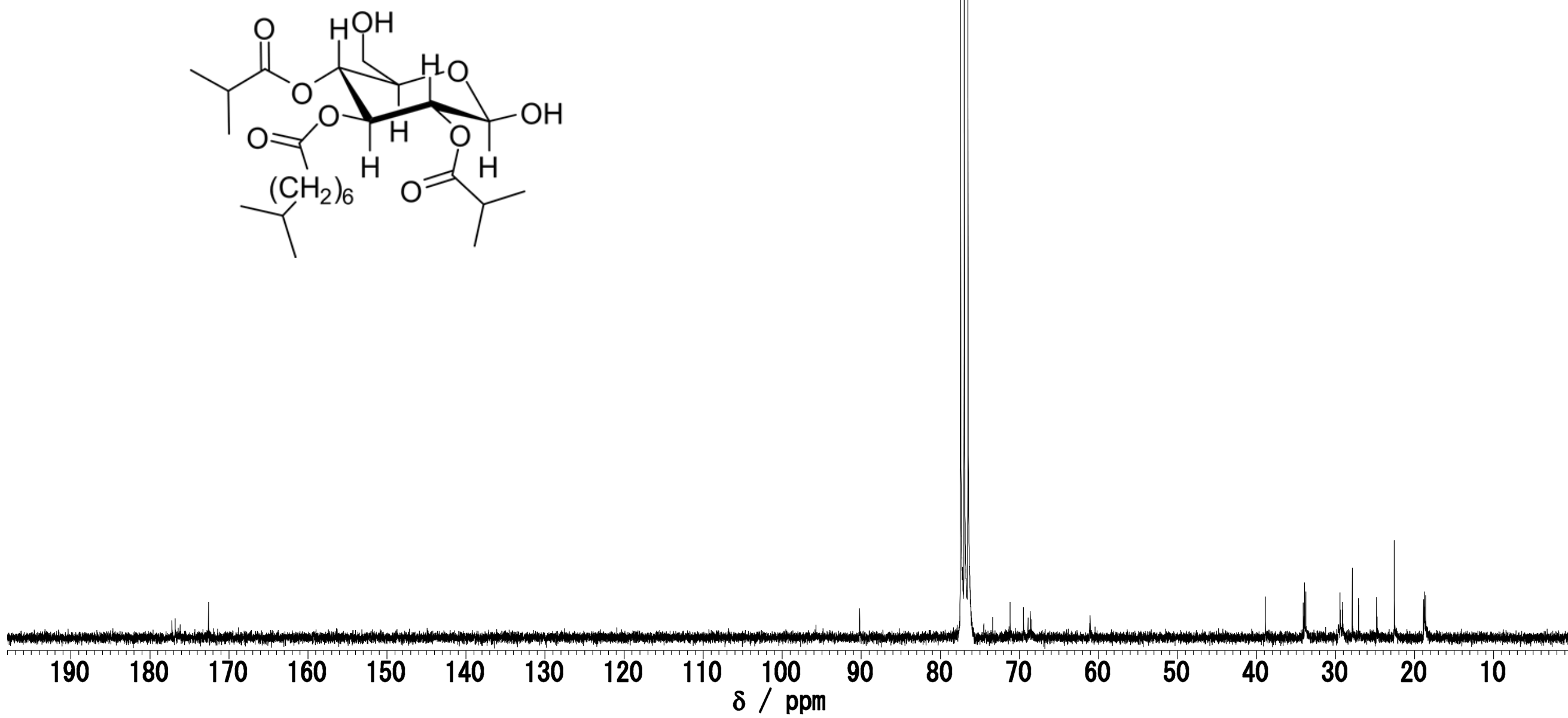

Figure S37. ${ }^{13} \mathrm{C}$ NMR spectrum of $2\left(270 \mathrm{MHz}, \mathrm{CDCl}_{3}\right)$. 

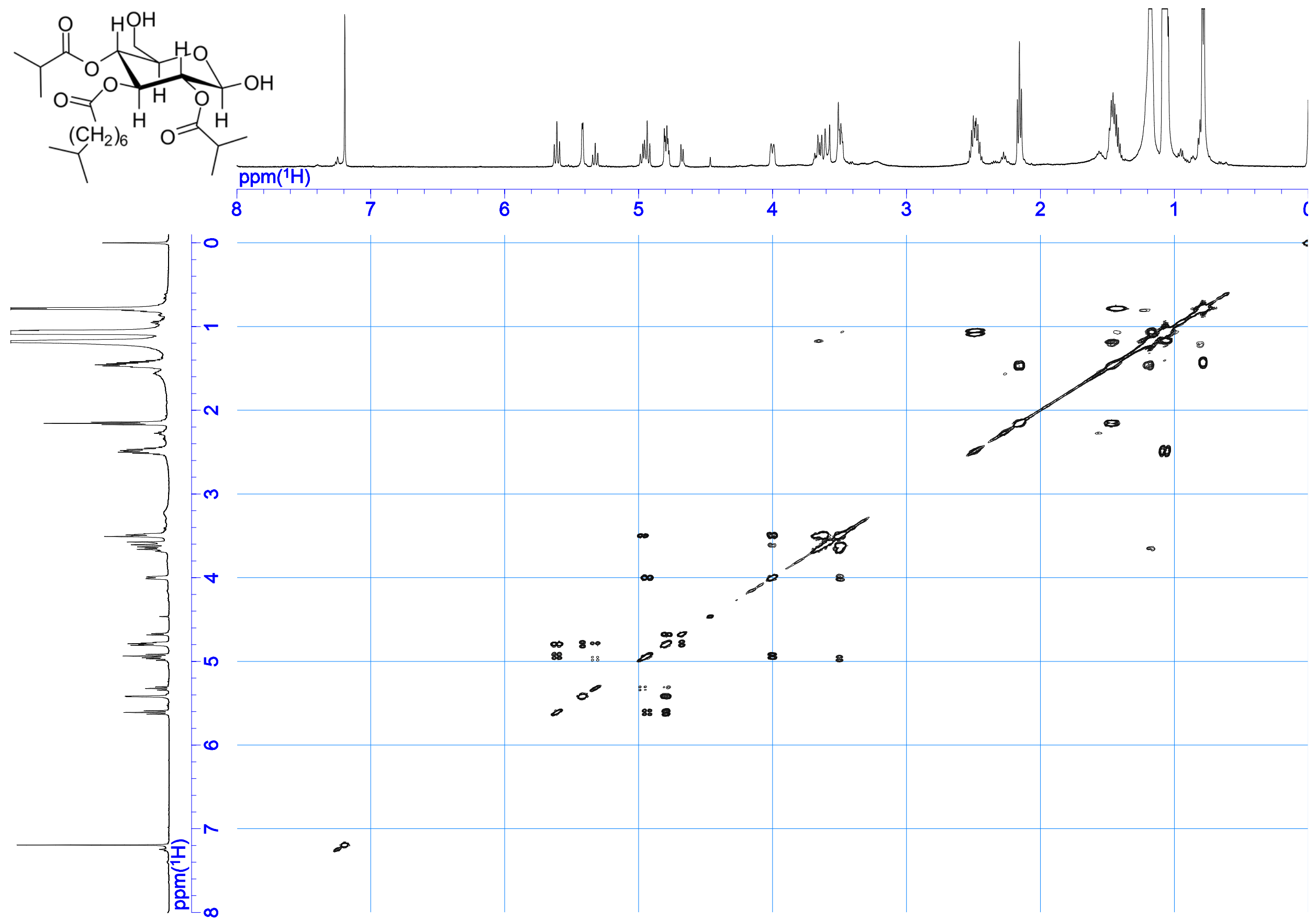

Figure S38. COSY spectrum of $2\left(500 \mathrm{MHz}, \mathrm{CDCl}_{3}\right)$. 

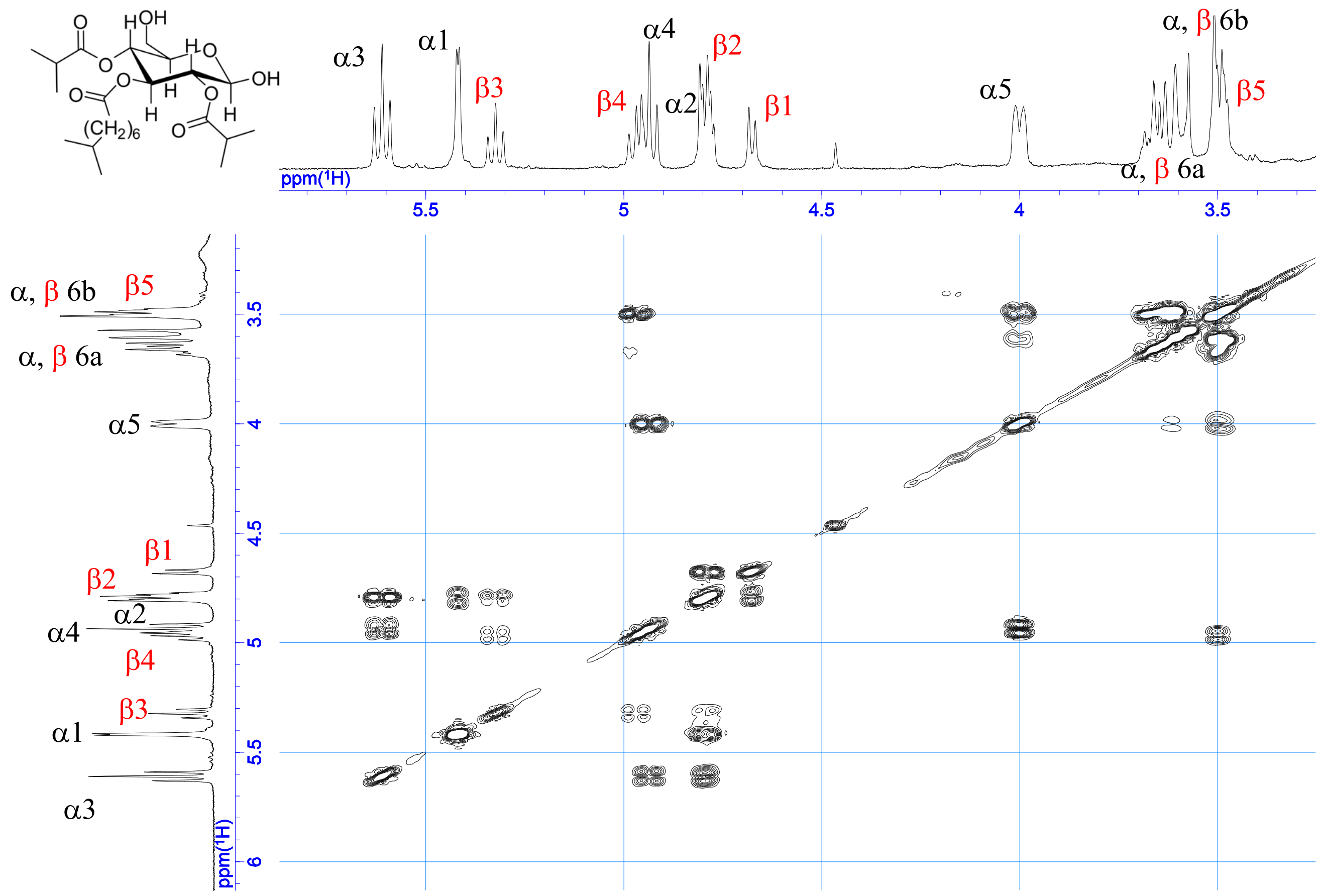

Figure S39. Partial COSY spectrum of $2\left(500 \mathrm{MHz}, \mathrm{CDCl}_{3}\right)$. 

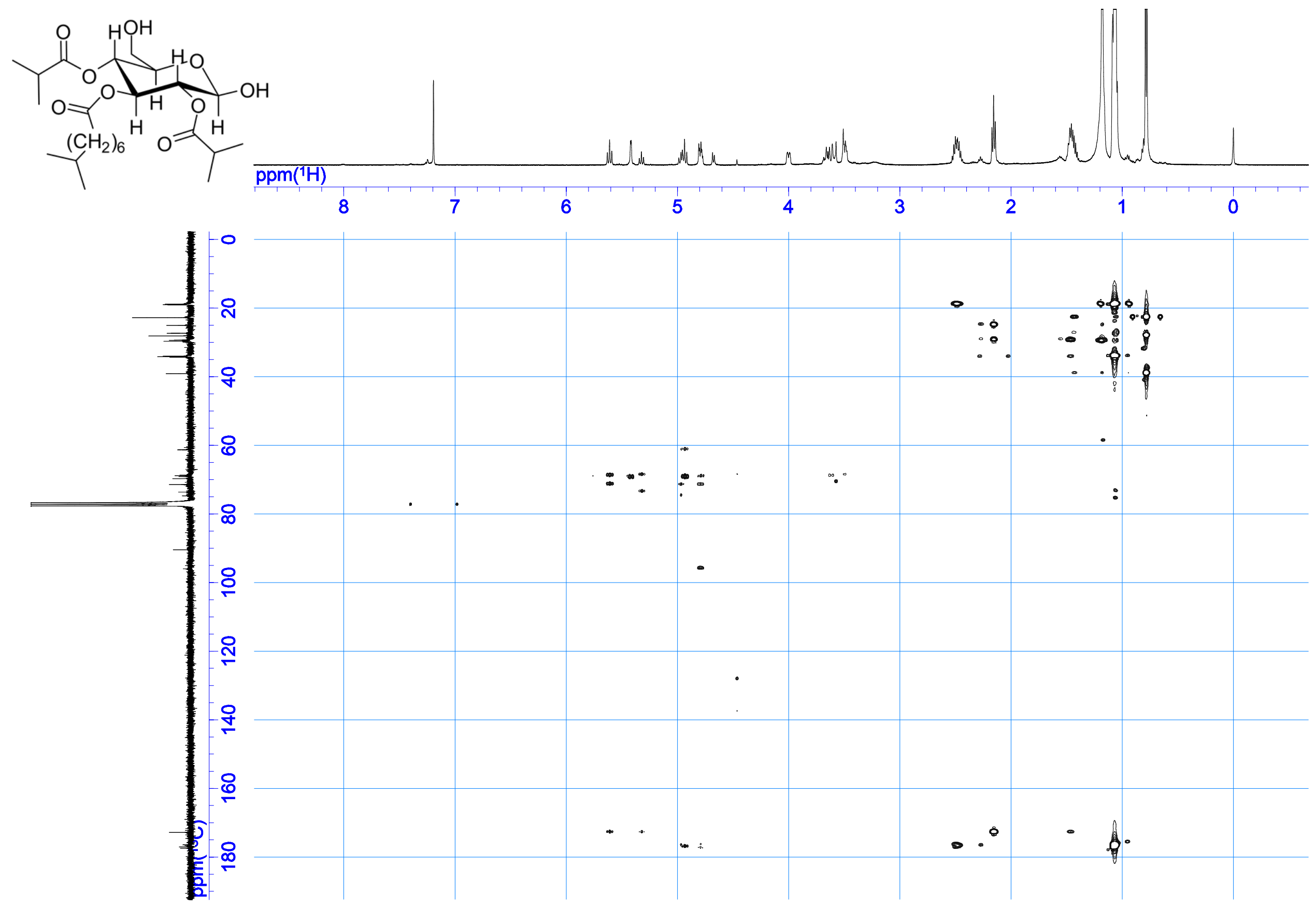

Figure S40. HMBC spectrum of $2\left(500 \mathrm{MHz}, \mathrm{CDCl}_{3}\right)$. 


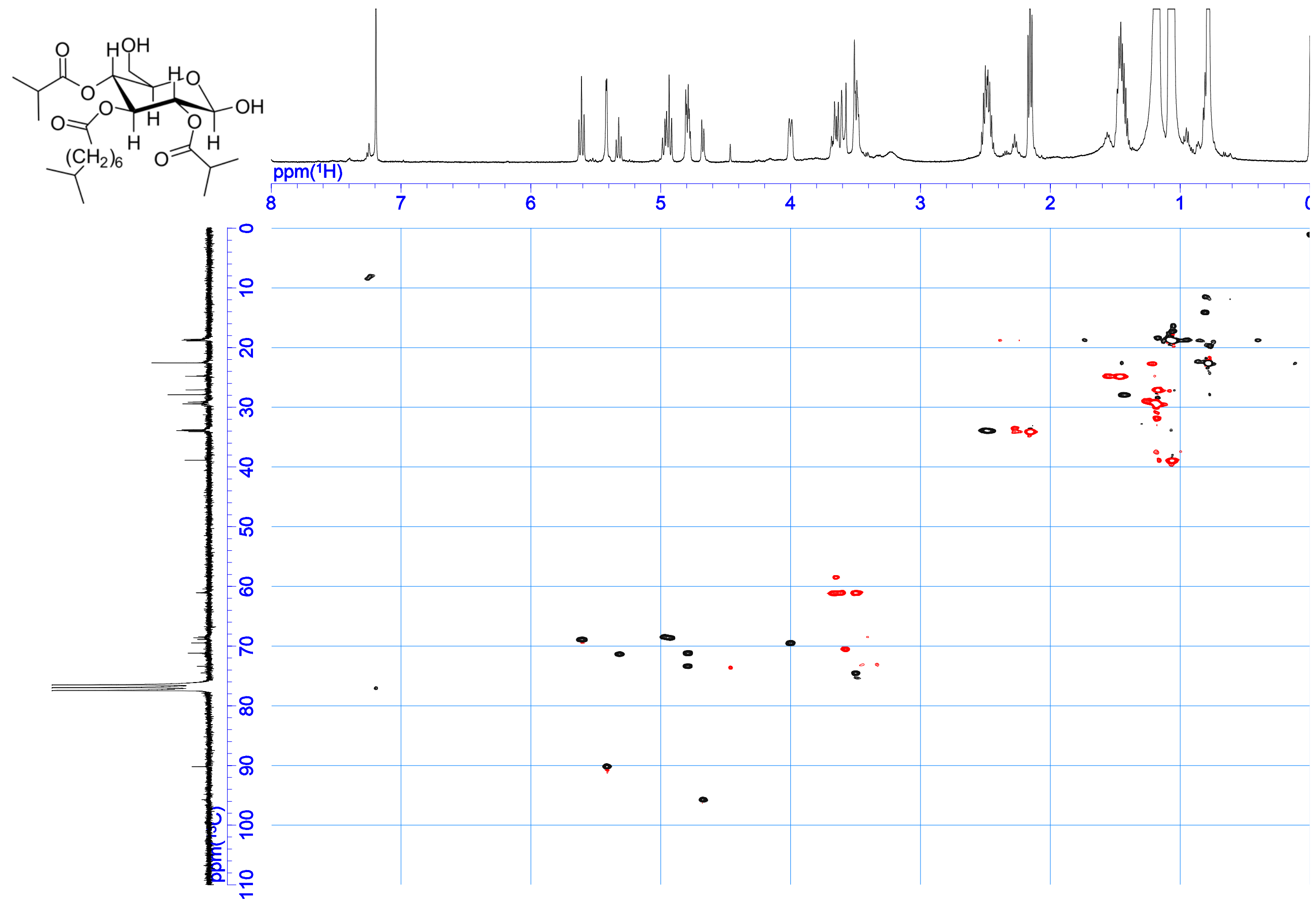

Figure S41. HMQC spectrum of $2\left(500 \mathrm{MHz}, \mathrm{CDCl}_{3}\right)$. 

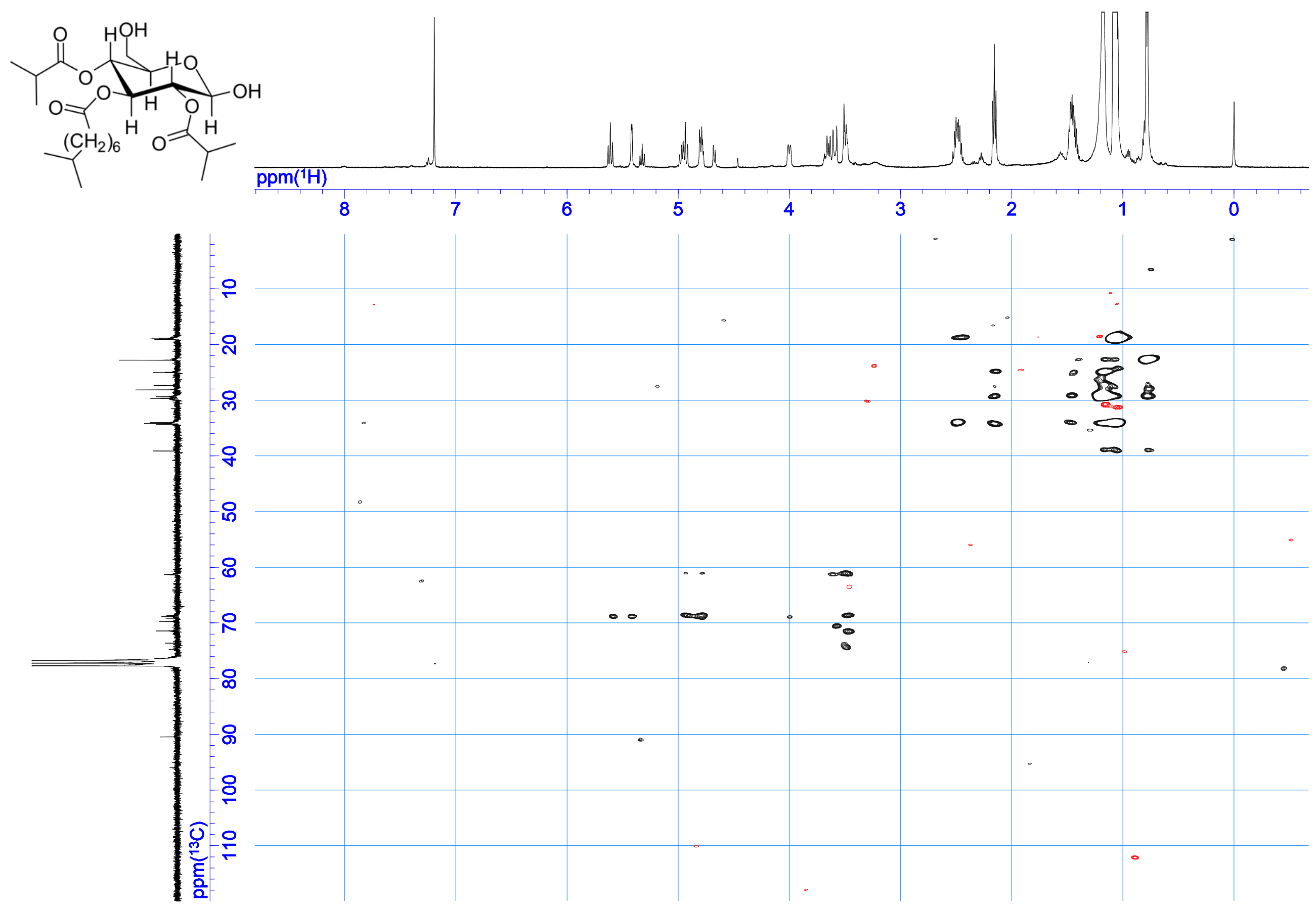

Figure S42. HSQC-TQCSY spectrum of $2\left(500 \mathrm{MHz}, \mathrm{CDCl}_{3}\right)$. 


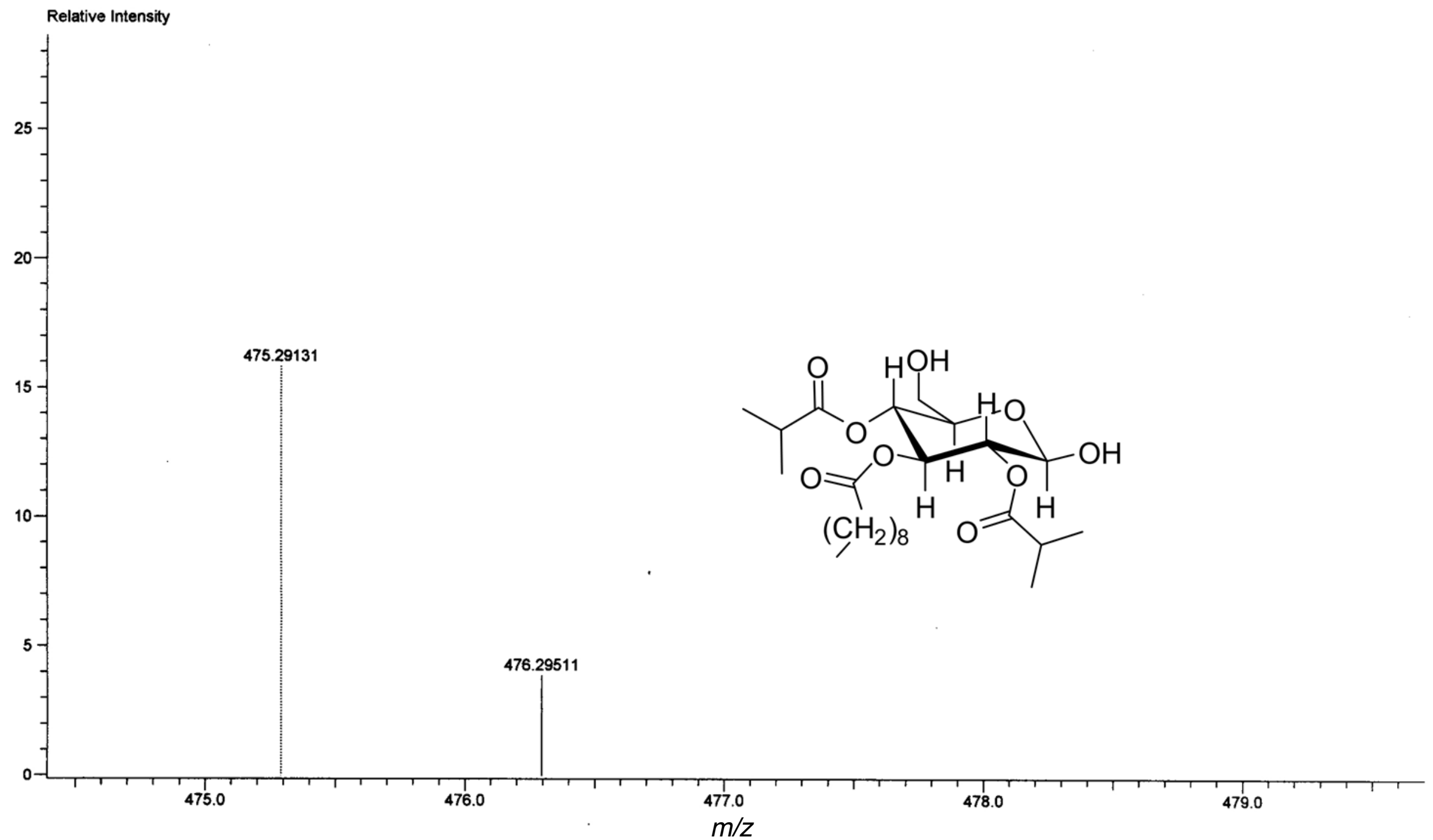

Figure S43. HRFD-MS spectrum of 3. 


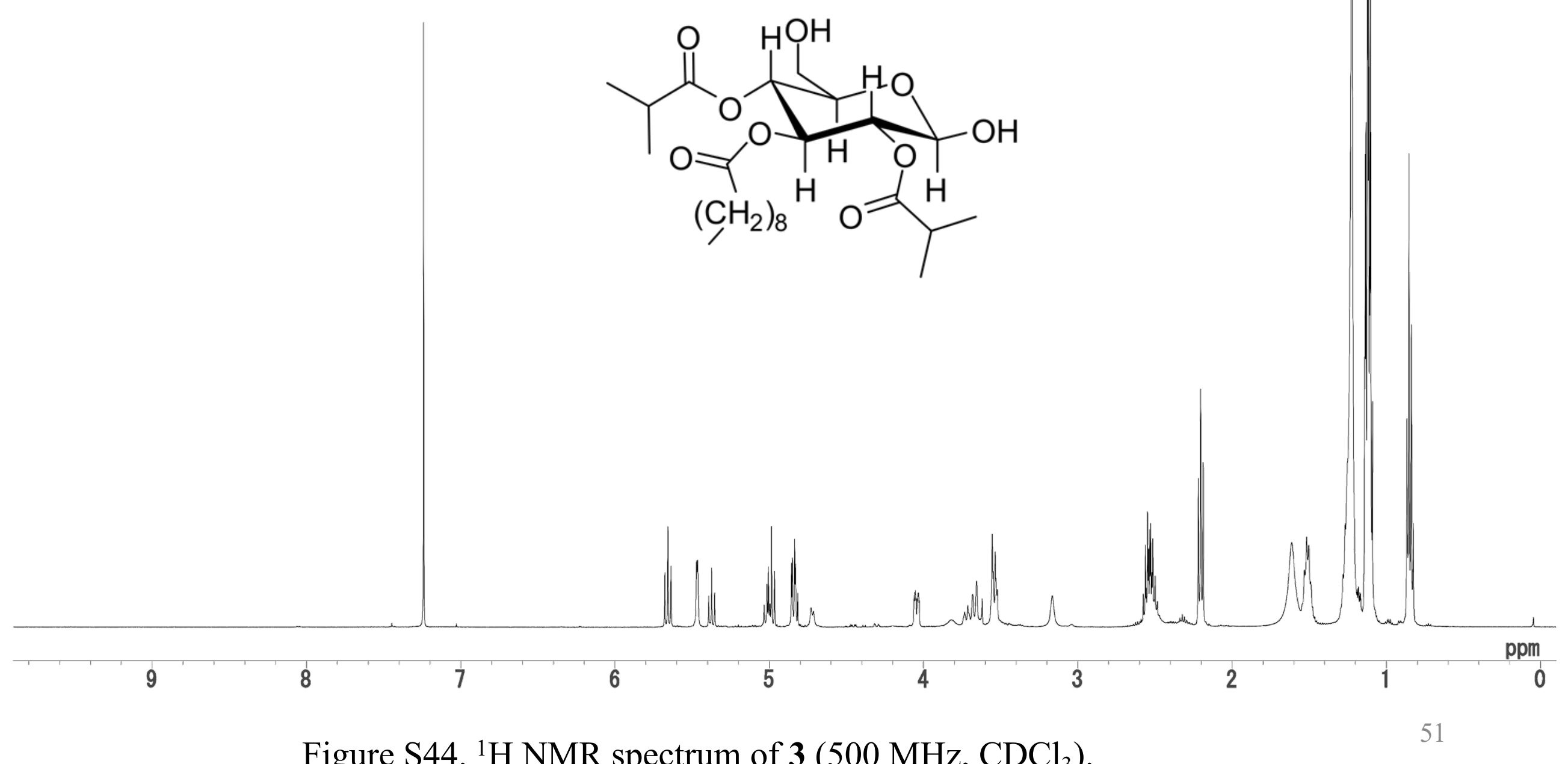

Figure S44. ${ }^{1} \mathrm{H}$ NMR spectrum of $3\left(500 \mathrm{MHz}, \mathrm{CDCl}_{3}\right)$. 


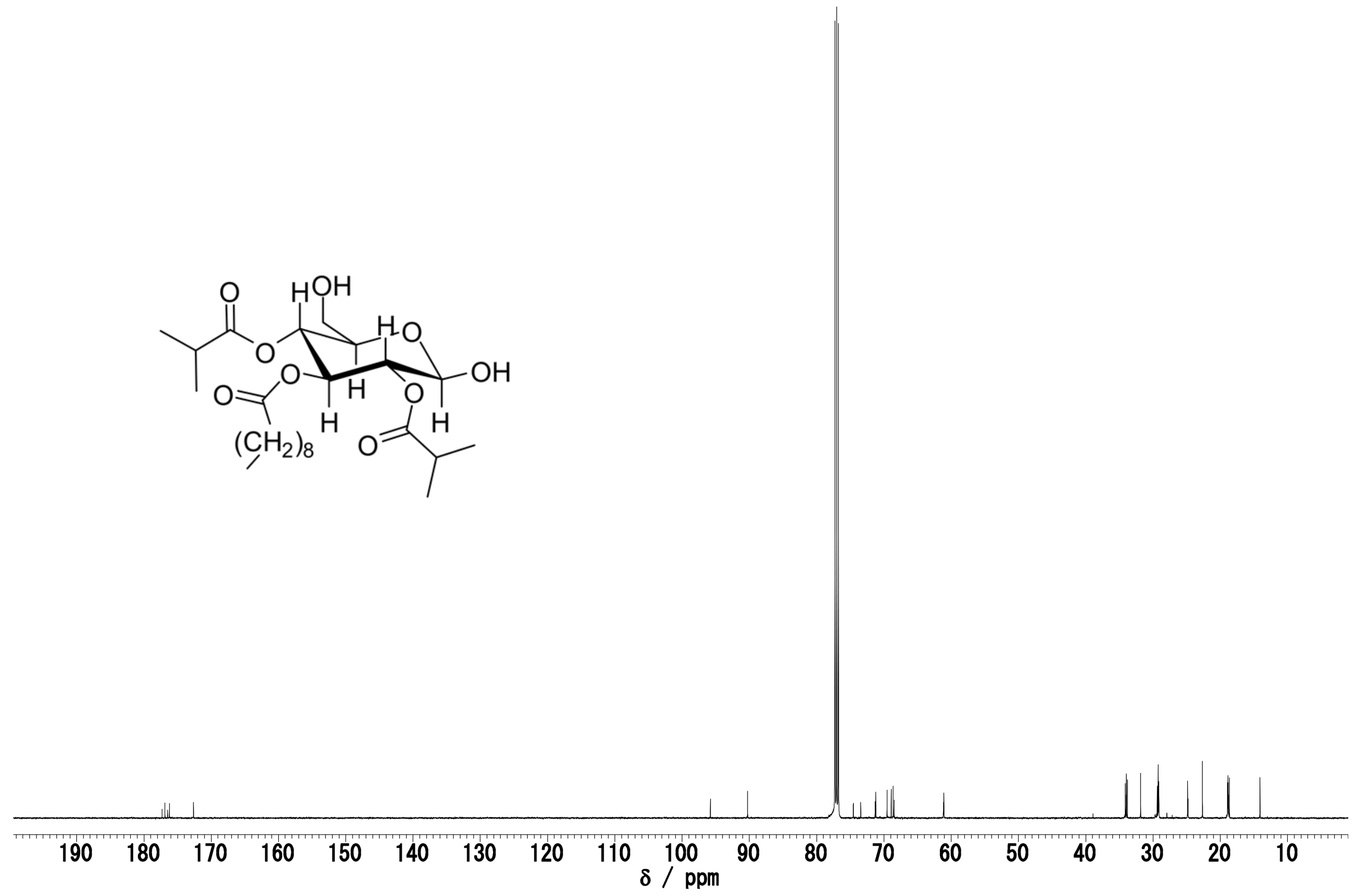

Figure $\mathrm{S} 45 .{ }^{13} \mathrm{C}$ NMR spectrum of $3\left(500 \mathrm{MHz}, \mathrm{CDCl}_{3}\right)$. 

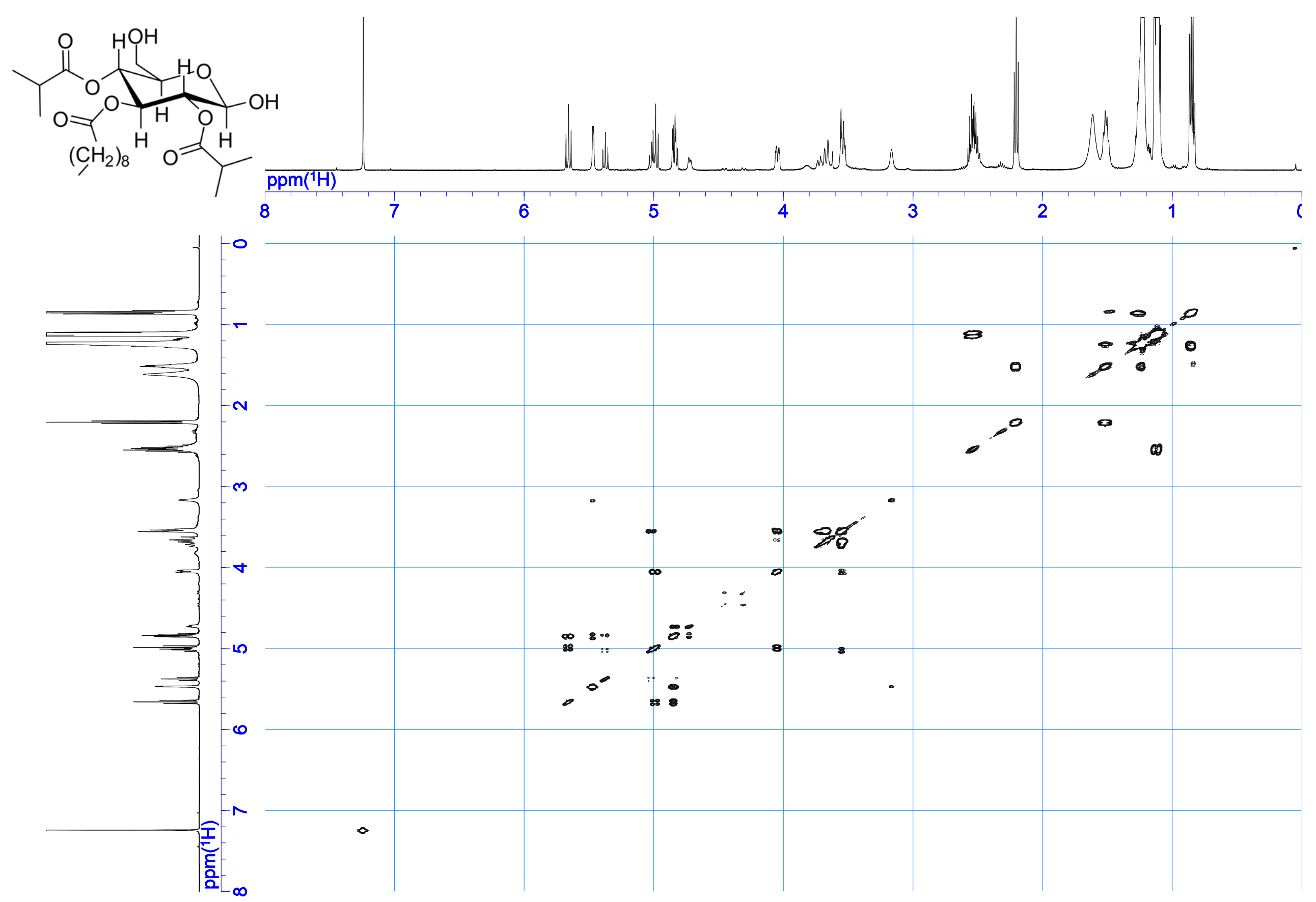

Figure S46. COSY spectrum of $3\left(500 \mathrm{MHz}, \mathrm{CDCl}_{3}\right)$. 

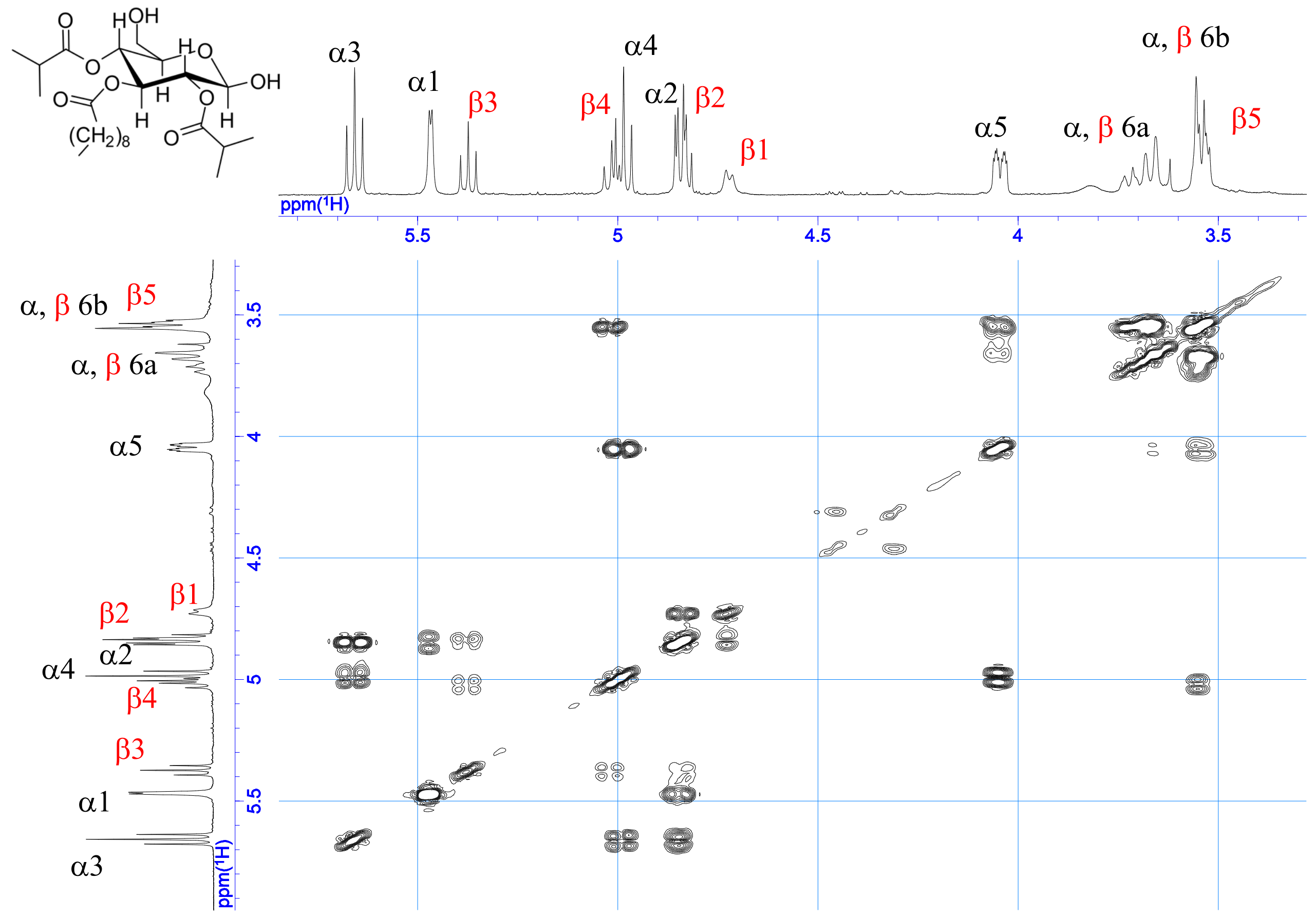

Figure S47. Partial COSY spectrum of $3\left(500 \mathrm{MHz}, \mathrm{CDCl}_{3}\right)$. 

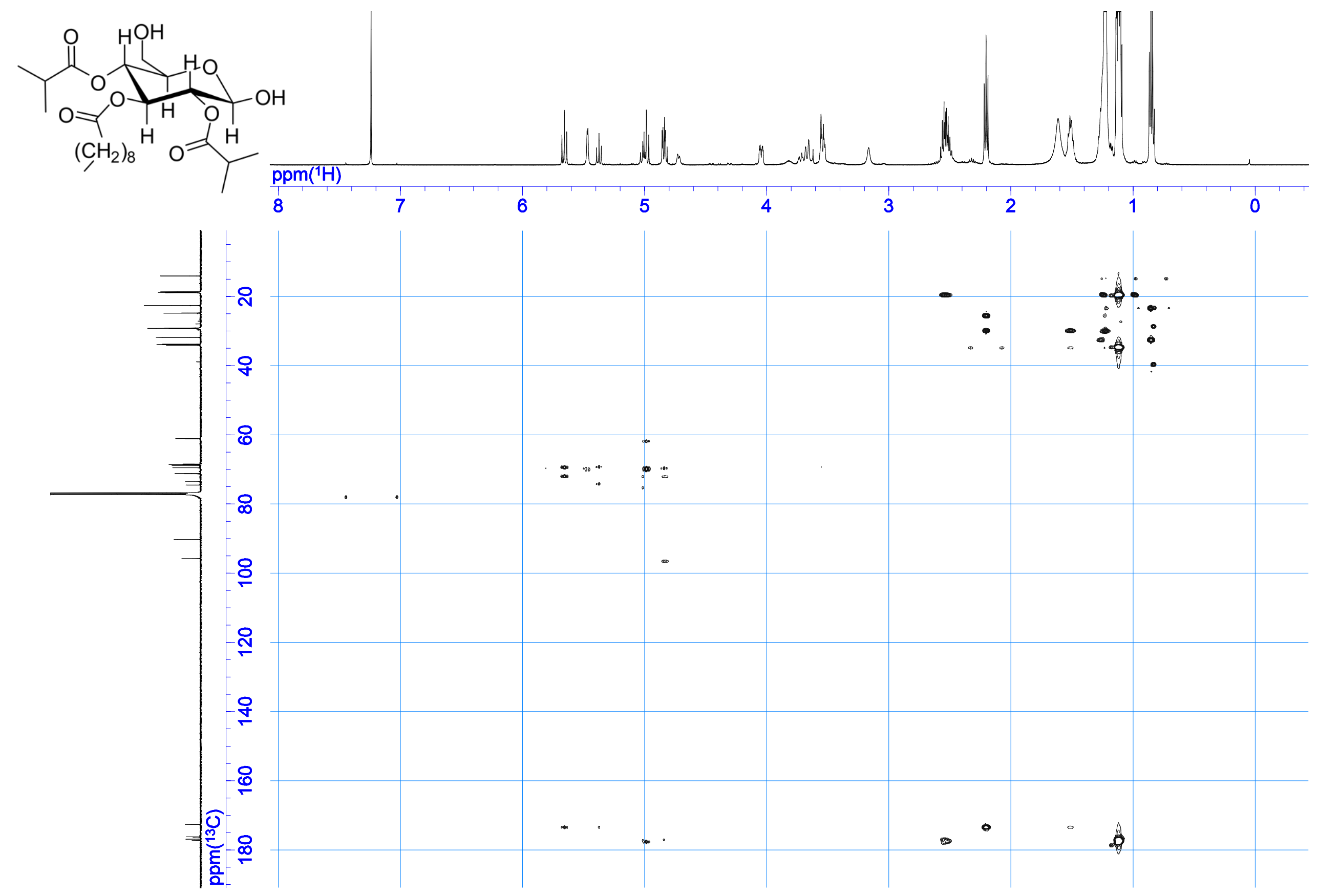

Figure S48. HMBC spectrum of $\mathbf{3}\left(500 \mathrm{MHz}, \mathrm{CDCl}_{3}\right)$. 

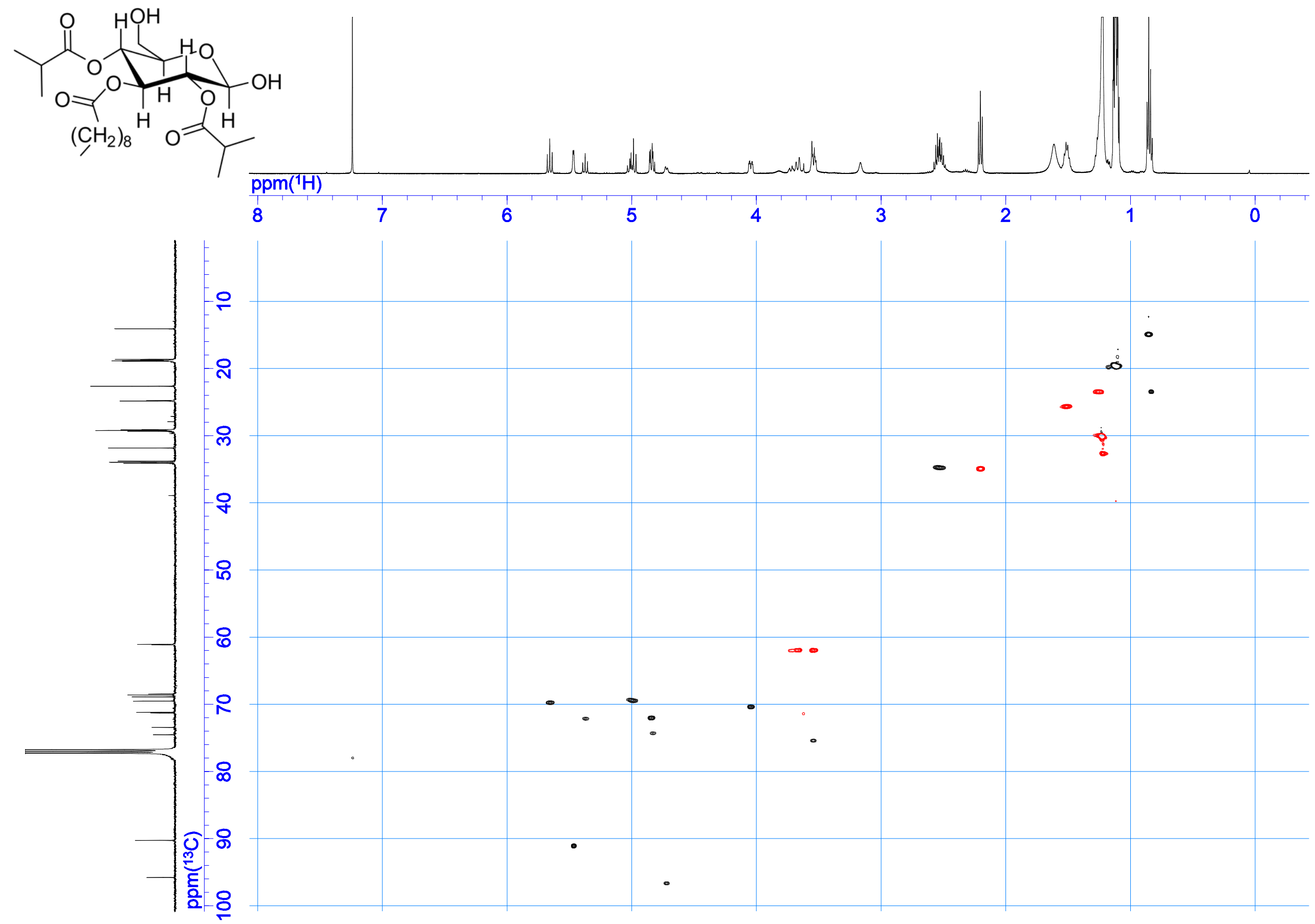

Figure S49. HMQC spectrum of $3\left(500 \mathrm{MHz}, \mathrm{CDCl}_{3}\right)$. 

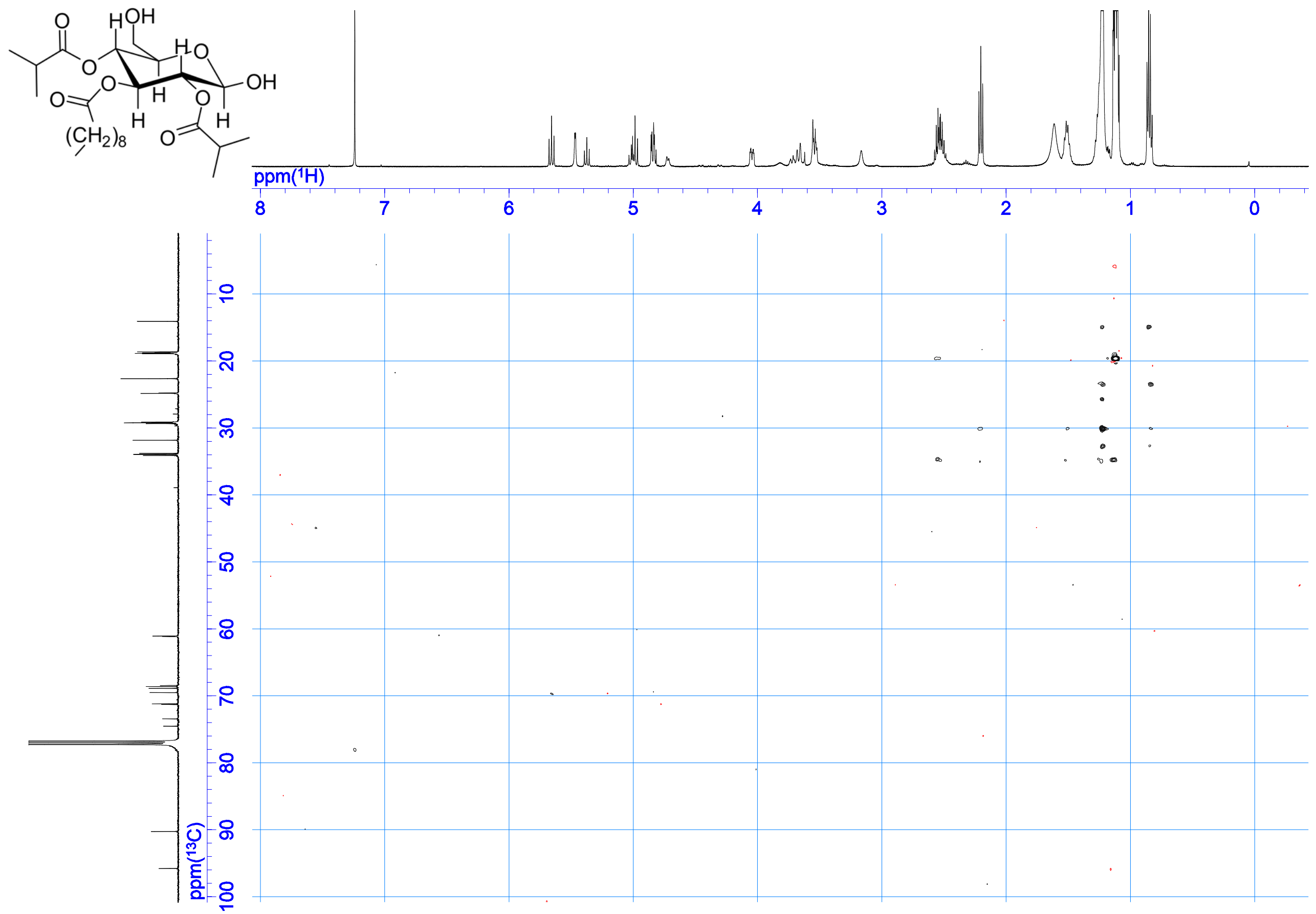

Figure S50. HSQC-TQCSY spectrum of $\mathbf{3}\left(500 \mathrm{MHz}, \mathrm{CDCl}_{3}\right)$. 


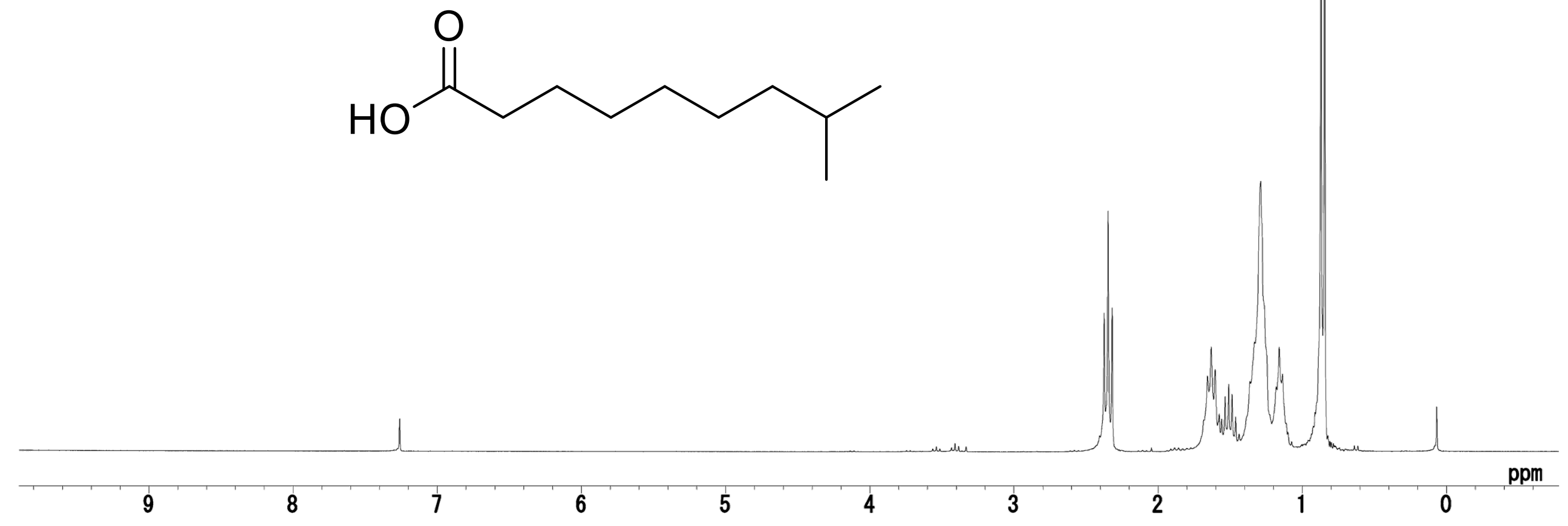

Figure S51. ${ }^{1} \mathrm{H}$ NMR spectrum of 8-methylnonanoic acid (9) $\left(270 \mathrm{MHz}, \mathrm{CDCl}_{3}\right)$. 


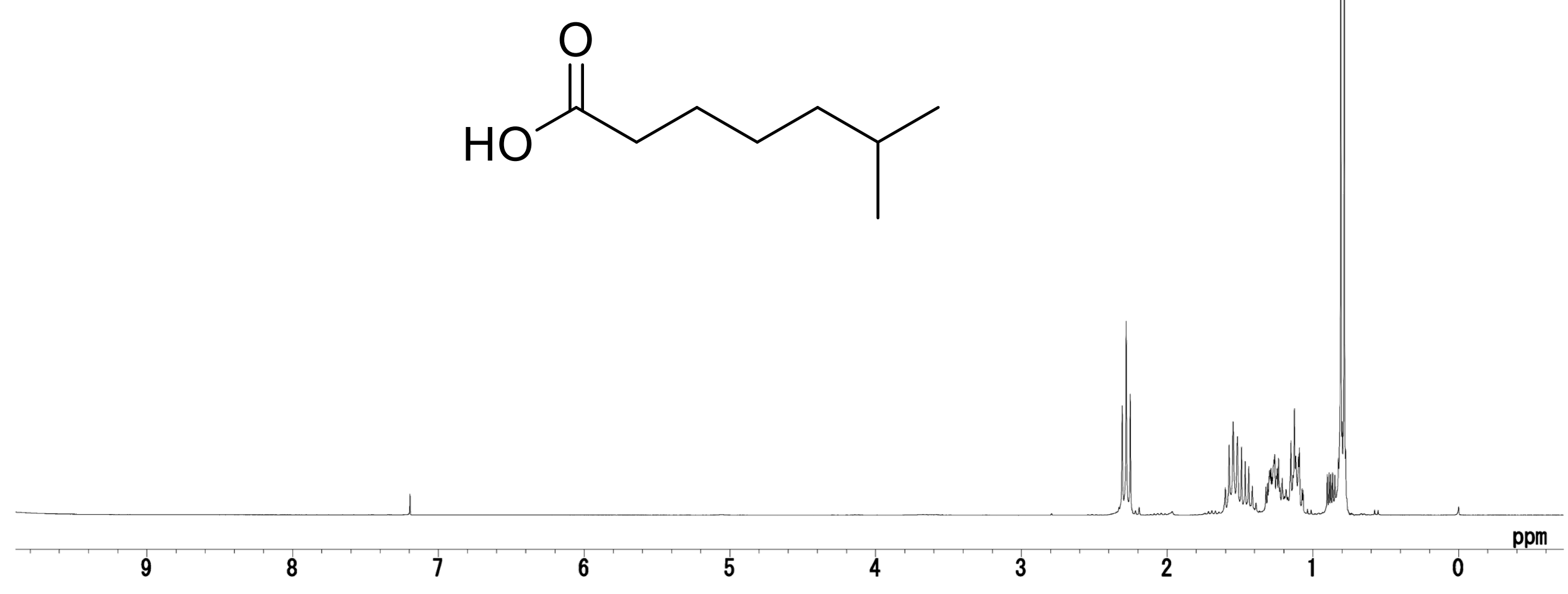

Figure S52. ${ }^{1} \mathrm{H}$ NMR spectrum of 6-methylheptanoic acid (14) $\left(270 \mathrm{MHz}, \mathrm{CDCl}_{3}\right)$. 


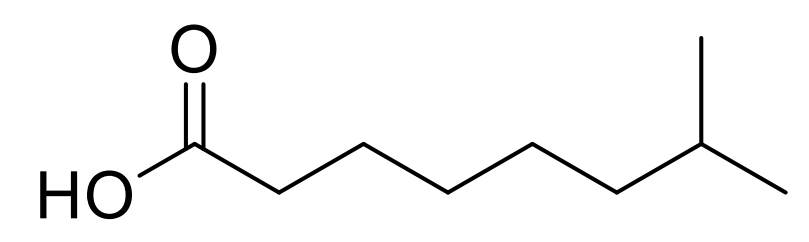

Figure S53. ${ }^{1} \mathrm{H}$ NMR spectrum of 7-methyloctanoic acid (15) $\left(270 \mathrm{MHz}, \mathrm{CDCl}_{3}\right)$. 